\title{
Elliptical instability of compressible flow and dissipation in rocky planets for strong tidal forcing
}

\author{
Dissertation \\ zur Erlangung des mathematisch-naturwissenschaftlichen \\ Doktorgrades \\ "Doctor rerum naturalium" \\ der Georg-August-Universität Göttingen \\ im Promotionsprogramm ProPhys \\ der Georg-August University School of Science (GAUSS)
}

vorgelegt von

Niels Clausen

aus Niebüll

Göttingen, 2015 


\section{Betreuungsausschuss}

Prof. Dr. Andreas Tilgner,

Institut für Geophysik, Universität Göttingen

Prof. Dr. Stefan Dreizler,

Institut für Astrophysik, Universität Göttingen

\section{Mitglieder der Prüfungskommission}

Referent: Prof. Dr. Andreas Tilgner,

Institut für Geophysik, Universität Göttingen

Korreferent: Prof. Dr. Stefan Dreizler,

Institut für Astrophysik, Universität Göttingen

\section{Weitere Mitglieder der Prüfungskommission}

Prof. Dr. Martin Rein,

Institut für Aerodynamik und Strömungstechnik, DLR

Prof. Dr. Wolfgang Glatzel,

Institut für Astrophysik, Universität Göttingen

Prof. Dr. Wolfram Kollatschny,

Institut für Astrophysik, Universität Göttingen

Prof. Dr. Gert Lube,

Institut für Numerische und Angewandte Mathematik, Universität Göttingen

Tag der mündlichen Prüfung: 16. Dezember 2015 


\section{Contents}

$\begin{array}{ll}\text { Nomenclature } & 5\end{array}$

$\begin{array}{ll}\text { Scientific contribution } & 9\end{array}$

$\begin{array}{ll}\text { 1. Introduction } & 11\end{array}$

2. Elliptical instability of compressible flow in ellipsoids 15

2.1. Theoretical background . . . . . . . . . . . . . . . 17

2.1.1. Mathematical formulation of the model . . . . . . . . . . . 17

2.1.2. Librational driven elliptical instability . . . . . . . . . . . . . . . . . . . . . . . . . . . . . . . . .

2.2. Numerical implementation . . . . . . . . . . . . . . . . . . . . . . 28

2.3. Results . . . . . . . . . . . . . . . . . . . . . . . . . . . 29

2.3.1. Examples: Io's tides on Jupiter, the binary system V636 Centauri and the Earth . . . . . . . . . . . . . . . . . . . . . . . . . . . . . . .

2.4. Conclusion and discussion . . . . . . . . . . . . . . . . . . 42

3. Dissipation of rocky planets for strong tidal forcing 43

3.1. Theoretical background . . . . . . . . . . . . . . 45

3.1.1. Equilibrium tide . . . . . . . . . . . . . . 45

3.1.2. Heat transport mechanism . . . . . . . . . . . . . . . 52

3.1.3. Coupling between heat transport and tidal heat production . . . . . 57

3.1.4. Rheology . . . . . . . . . . . . . . . . . . 59

3.1.5. Tidal quality factor . . . . . . . . . . . . . . . 6 60

3.2. Validation . . . . . . . . . . . . . . . . . 60

3.3. Results . . . . . . . . . . . . . . . . . . . 6 61

3.3.1. Tidal dissipation of $\mathrm{Io} \ldots \ldots$. . . . . . . . . . . . . . . . . . . . . . . . 61

3.3.2. Tidal dissipation of Corot $7 \mathrm{~b} \ldots \ldots$. . . . . . . . . 68

3.4. Conclusion and discussion . . . . . . . . . . . . . . . . . 73 
4. Inertial modes in shear flow 75

4.1. Equations for fluid motion in Ekman-Couette flow . . . . . . . . . . . . 76

4.2. Results . . . . . . . . . . . . . . . . . . . . 78

4.3. Conclusion and discussion . . . . . . . . . . . . . . . . . . 82

5. Summary 85

A. Eigenvalue problem for the power-law density sphere 89

B. Orthogonality of the scalar product 93

C. Relation between frequency difference and ellipticity 94

D. Equations governing spheroidal oscillations 96

E. Continuity of the total gravitational potential and its gradient 99

$\begin{array}{ll}\text { F. MAPLE commands } & 101\end{array}$

$\begin{array}{ll}\text { Bibliography } & 102\end{array}$

List of Figures 113

$\begin{array}{ll}\text { List of Tables } & 117\end{array}$ 


\section{Nomenclature}

Most symbols used in the work can be found in this nomenclature. We sort the symbols by the chapters in which they appear, global symbols can appear in all chapters.

\section{Global symbols}

$(r, \theta, \varphi)$

$\xi$

$\delta Z$

$\mathbf{u}$

$\hat{\mathbf{x}}, \hat{\mathbf{y}}, \hat{\mathbf{z}}$

$\Phi$

$\Phi_{\mathrm{t}}$

$\rho$

$G$

$L$

$p$

$Z^{\prime}$

$Z_{0}$ spherical coordinates

displacement vector

for any variable $\mathrm{Z}$ a preceding $\delta$ denotes it is a Lagrangian perturbation quantity

velocity vector

unit vectors in $\mathrm{x}, \mathrm{y}, \mathrm{z}$ direction

gravitational and possibly centrifugal potential

tidal potential

density

gravitational constant

stellar luminosity

pressure

for any variable $Z$ a prime denotes it is an Eulerian perturbation quantity, this prime will generally be omitted

for any variable $Z$, subscript zero denotes it is a background quantity

\section{Symbols used in Chap. 2}




\begin{tabular}{|c|c|}
\hline$\left(\bar{x}_{1}, \bar{x}_{2}, \bar{\varphi}\right)$ & ellipsoidal coordinates according to Bryan (1889) \\
\hline$<n>$ & $\begin{array}{l}\text { mean value of the spatial degree for a positive growth rate of the } \\
\text { elliptical instability for small } \Omega\end{array}$ \\
\hline$\beta$ & exponent for the background density profile \\
\hline$\epsilon$ & ellipticity of the ellipsoid \\
\hline$\iota$ & adiabatic index \\
\hline$\nu$ & kinematic viscosity \\
\hline$\Omega$ & $\Omega=\Omega_{\mathrm{p}} / \Omega_{\mathrm{F}}$ \\
\hline$\omega$ & frequency of the inertial mode in the frame rotating with $\Omega_{\mathrm{p}}$ \\
\hline$\Omega_{\mathrm{b}}(t)$ & $\begin{array}{l}\text { angular velocity of the tidal bulge in the frame that rotates with } \\
\text { the spin of the central object }\end{array}$ \\
\hline$\omega_{\mathrm{e}}$ & frequency of the elliptical instability \\
\hline$\Omega_{\mathrm{F}}$ & $\begin{array}{l}\text { angular velocity of the spin of the central body in the frame rotat- } \\
\text { ing with } \Omega_{\mathrm{p}}\end{array}$ \\
\hline$\Omega_{\mathrm{p}}$ & orbital angular velocity of the perturbing body in the inertial frame \\
\hline$\Omega_{\text {spin }}$ & angular velocity of the spin of the central body in the inertial frame \\
\hline$\Phi_{\text {self }}$ & $\begin{array}{l}\text { the potential terms created by the central body itself (gravitational } \\
\text { and centrifugal) }\end{array}$ \\
\hline$\psi$ & $\psi=\frac{p^{\prime}}{\rho_{0}}+\Phi^{\prime}$ \\
\hline$\sigma_{\mathrm{fs}}$ & $\begin{array}{l}\text { growth rate of the elliptical instability with free slip boundary } \\
\text { conditions }\end{array}$ \\
\hline$\sigma_{\text {inv }}$ & inviscid growth rate of the elliptical instability \\
\hline$\sigma_{\mathrm{ns}}$ & $\begin{array}{l}\text { growth rate of the elliptical instability with no slip boundary con- } \\
\text { ditions }\end{array}$ \\
\hline$\sigma_{\mathrm{ud}}$ & $\begin{array}{l}\text { maximal inviscid growth rate of the elliptical instability in an un- } \\
\text { bounded domain }\end{array}$ \\
\hline$\tilde{\epsilon}$ & strain rate \\
\hline$\varpi_{1}$ & the amplitude of libration \\
\hline$\zeta$ & $\zeta=-q / 2$ \\
\hline
\end{tabular}




$\begin{array}{ll}a, b, c & \text { ellipsoidal semi major axes } \\ c_{\mathrm{s}} & \text { speed of sound } \\ E k & \text { Ekman number } \\ f & f=\omega_{\mathrm{e}}+i \sigma_{\mathrm{inv}} \\ h_{1} & \text { constant in the volume damping term to calculate } \sigma_{\mathrm{ns}} \text { and } \sigma_{\mathrm{fs}} \\ h_{2} & \text { constant in the boundary damping term to calculate } \sigma_{\mathrm{ns}} \\ k & \text { azimuthal wavenumber of the inertial mode } \\ m & \text { a number corresponding to the frequency of the inertial mode } \\ n & \text { spatial degree of the inertial mode } \\ n_{\text {min }} & \text { minimal spatial degree necessary for a positive growth rate of the } \\ N_{\mathrm{p}} & \text { elliptical instability for large } \Omega \\ q & \text { polytropic index } \\ \text { frequency of the inertial mode in the frame rotating with } \Omega_{\mathrm{p}}+\Omega_{\mathrm{F}}\end{array}$

\section{Symbols used in Chap. 3}

$\bar{R}$

$\eta$

$\gamma$

$\mu$

$\theta$

$a_{\mathrm{c}}, \alpha, \varsigma$

$B$

$b$

$d_{c}$

$e$

$E_{a}$

F

$g$ universal gas constant

dynamic viscosity

parameter defined as $\gamma=E_{a} /\left(\bar{R} T_{i}^{2}\right)$

shear modulus

Frank-Kamenetskii parameter $\theta=\gamma\left(T_{i}-T_{\mathrm{S}}\right)$

constants depending on the chosen $N u(R a)$ relation, see Eq. (3.51)

dimensionless melt fraction coefficient

grain size

thickness of the convecting layer

eccentricity of the orbit

activation energy for subsolidus mantle creep

heat flux

gravitational acceleration 


$\begin{array}{ll}H & \text { rate of internal heat generation }\left(\mathrm{in} \mathrm{W} / \mathrm{m}^{3}\right) \\ k_{2} & \text { tidal love number } \\ K_{\mathrm{E}} & \text { bulk modulus } \\ N u & \text { Nusselt number } \\ P & \text { orbital period } \\ Q & \text { tidal quality factor, definition see Sect. 3.1.5 } \\ Q^{\prime} & \text { modified tidal quality factor, definition see Sect. 3.1.5 } \\ R_{c} & \text { radius of the core of the object } \\ R_{p} & \text { radius of the object } \\ R a & \text { Rayleigh number } \\ T_{\mathrm{i}} & \text { interior temperature } \\ T_{\mathrm{S}} & \text { surface temperature }\end{array}$




\section{Scientific contribution}

N. Clausen and A. Tilgner. Elliptical instability of compressible flow in ellipsoids. $A \mathscr{E} A$, 562:A25, 2014

N. Clausen and A. Tilgner. Dissipation in rocky planets for strong tidal forcing. $A \& A$, 584:A60, 2015

Parts of these articles are reproduced in this thesis with permission OESO. 


\section{Introduction}


The investigation of tidal friction has been a subject of research for many years (e.g. Darwin 1879). This study aims to serve as a further building block in the understanding of tidal friction, which causes the circularization and synchronization of stars, planets, and moons on their orbit around their tidal partner. A famous example of this is the Earth-Moon system. The Moon's rotational velocity around its own axis is synchronized with its orbital rotational velocity; one therefore says that the Moon is tidally locked. As a consequence, one face of the Moon cannot be seen from the Earth. However, due to the Moon's libration, caused by the ellipticity of the orbit, we can see at least $59 \%$ of the Moon's surface from the Earth (Chu et al., 2012). Unlike the Moon's spin, that of Earth is not synchronized with its orbital angular velocity; it spins faster than the Moon rotates around the Earth, resulting in a slow drifting away of the Moon from the Earth and a decreasing rotation rate of Earth's spin (see, e.g., Murray and Dermott 1999). This is different from many other systems in which the satellite is tidally locked and the host object spins slower than the satellite ${ }^{1}$ rotates around it, which leads to a decrease in the semi-major axis. A famous example is the hot-Jupiter planet WASP18b: "Under the assumption that the tidal-dissipation parameter $Q^{2}$ of the host star is of the order of $10^{6}$, as measured for Solar System bodies and binary stars and as often applied to extrasolar planets, WASP-18b will be spiraling inwards on a timescale less than a thousandth that of the lifetime of its host star." (Hellier et al., 2009). This citation illustrates the previously conventional practice of simply applying a dissipation factor of $10^{5}-10^{6}$ to a star. This stellar $Q^{\prime}$ value coincides with studies of binary star systems (Meibom and Mathieu, 2005), but there is disagreement on whether this is the correct way to parameterize tidal dissipation. In an exoplanet population study, Penev et al. (2012) find that the stellar $Q^{\prime}$ is greater than $10^{7}$ at the 99 percent confidence level. They then argue that this disagreement between the $Q^{\prime}$ s arises from the tidal frequency being much higher than the stellar rotation in the case of an exoplanet inspiral. Conversely, in a binary star system the tidal frequency is generally close to the rotation of the star, due to a faster synchronization (see also Penev and Sasselov 2011). Thus, damping due to inertial modes can occur in the latter case, whereas in the former case turbulent dissipation by the static tide - which is less efficient - is probably the dominant dissipation mechanism. Inertial modes in a star can get resonantly excited by the tidal perturbation if the spin period of the star is less than twice the orbital period of its satellite, because the absolute value of the frequency of an inertial mode in a spinning object is always less then twice the spin frequency (see, e.g., Greenspan 1968). The results of Penev et al. (2012) highlight the importance of knowing the energy dissipating mechanism. A review of the different tidal dissipation mechanisms for binary stars can be found, e.g., in Rieutord (2003). A newer

\footnotetext{
${ }^{1}$ In this study a satellite can be a moon that orbits a planet as well as a planet that orbits a star.

${ }^{2}$ What Hellier et al. (2009) call $Q$ here is equivalent to our $Q^{\prime}$; they use the definition as in Jackson et al. (2008) for $Q$. In the following we call it therefore $Q^{\prime}$. For our definition of $Q$ and $Q^{\prime}$ see Sect. 3.1.5.
} 
potential mechanism is the elliptical instability, which is investigated in the first part of this study, Chap. 2.

Similar to the proceeding for the $Q^{\prime}$ value of a star is the proceeding for the $Q^{\prime}$ value of planets. For a terrestrial planet, one often calculates with a modified tidal dissipation factor $Q^{\prime}$ of 100 , which is on the order of the Earth's $Q^{\prime}$ value (Murray and Dermott, 1999). In Chap. 3 we will take a closer look at whether this proceeding is appropriate for rocky planets under strong tidal forcing.

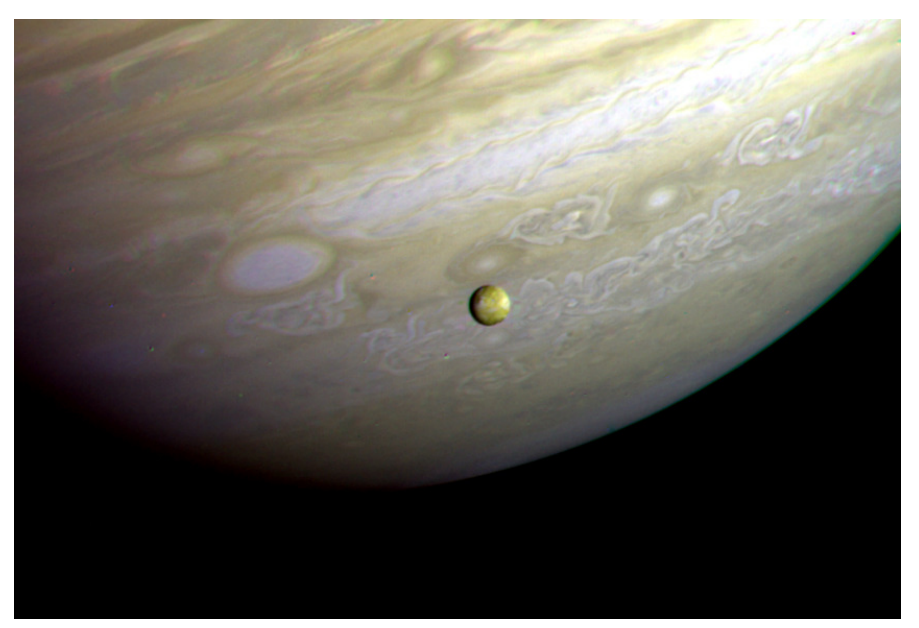

Figure 1.1.: Jupiter and Io photographed by the Voyager 2 probe on 9 July 1979. Courtesy: NASA

Figure 1.1 illustrates the common significance of the various investigations in this study. Jupiter is a gas giant in which elliptical instability may play a role in the tidal dissipation process, and Io is a Jovian moon under strong tidal forcing.

\section{This thesis}

In the first part of this study, Chap. 2, we investigate the elliptical instability. We calculate the growth rates of the elliptical instability under the influence of compressibility in a slightly elliptical deformed sphere. To do so, we solve the linearized Euler equations; the viscosity is included heuristically. The use of a power law for the radial dependence of the density and the anelastic approximation makes it possible to use semi-analytical methods to solve the equations. The influence of the orbital frequency of the perturber is considered. Exemplarily we apply the results to decide if elliptical instability is possible in the Earth perturbed by the Moon, Jupiter perturbed by Io and in the binary star system V636 Centauri. This chapter is based on the work by Clausen and Tilgner (2014). 
In Chap. 3 we present calculations of the tidal dissipation in rocky planets under strong tidal forcing. We estimate tidal dissipation by an equilibrium between heat transport and heat production due to tides, as done by, e.g., Moore (2003). The influence of convection as opposed to melt migration as a possible heat transport mechanism is investigated, as well as dissipation in a homogeneous mantel as opposed to dissipation occurring mainly in the asthenosphere. We use Jupiter's moon Io and the exoplanet Corot-7b as objects of study. This chapter is based on the work by Clausen and Tilgner (2015).

In Chap. 4 we solve the inviscid equations for inertial modes in shear flow, numerically for a profile with a velocity jump and analytically for a similar profile that has a continuous change of velocity instead of a jump. Our expectation is that with an appropriate steepness of the gradient in the continuous case, the flow profiles will approximately agree. We compare the resulting profiles to determine whether our expectation is fulfilled.

\section{Relation between this thesis and the published articles}

The work for the two publications (Clausen and Tilgner, 2014, 2015) on which this thesis is based was carried out by the author of this thesis. The wordings in the publications were partly written together with his supervisor. Large parts of this work were taken from the two publications (Clausen and Tilgner, 2014, 2015). Therefore, the major differences between the publications and this thesis must be pointed out.

Chapter 2 corresponds to Clausen and Tilgner (2014), although Section 2.1.2 is not from Clausen and Tilgner (2014). In Sect. 2.3.1, a new scaling for the turbulent viscosity in rotating convection according to Barker et al. (2014) is considered, which was published after the publication date of our article.

Chapter 3 corresponds to Clausen and Tilgner (2015). Section 3.1.1 includes the reformulation of the tidal potential, which simplifies the following derivation of the governing equations for spheroidal oscillations; neither the reformulation nor the derivation is from Clausen and Tilgner (2015). Furthermore, in this thesis in Sect. 3.1.1 the justification is given why it is appropriate to neglect the compressibility and the inertial term in the equations governing spheroidal oscillations. 
2 Elliptical instability of compressible flow in ellipsoids 
The elliptical instability was discovered in the mid-1970s (for a review, see Kerswell 2002). Elliptical instability is the name for an instability that arises in elliptical flows, because of a resonance between two inertial modes of the underlying rotating flow with the strain field. An elliptical flow is the superposition of a pure rotation and a stagnation point flow with stretching in the $-45^{\circ}$ direction. It can therefore be written as

$$
\mathbf{U}=\left(\begin{array}{ccc}
0 & -1-\tilde{\epsilon} & 0 \\
1-\tilde{\epsilon} & 0 & 0 \\
0 & 0 & 0
\end{array}\right)\left(\begin{array}{l}
x \\
y \\
z
\end{array}\right)=\hat{\mathbf{z}} \times \mathbf{x}+D \cdot \mathbf{x}
$$

where the vorticity is $2 \hat{\mathbf{z}}$ everywhere and $\tilde{\epsilon}$ is the strain rate, $\tilde{\epsilon}<1$ for elliptical flow, and $D$ is the strain matrix. Waleffe (1990) found that a simple solid body rotation $\mathbf{u}=\frac{1}{2} \boldsymbol{\omega} \times \mathbf{x}$ is an exact solution to the perturbed nonlinear Navier Stokes equation with the above background flow, with exponentially growing eigenmodes, due to vortex stretching. This simple vortex stretching mechanism is also the reason for the growing of the elliptical instability, under the prerequisite that certain resonance conditions are fulfilled. A tidally perturbed fluid body is elliptical deformed such that each fluid particle follows an elliptical streamline as long as the objects spin differs from it's orbital angular velocity with respect to the perturbing body. Therefore in not synchronized objects the elliptical instability can appear, but also for a synchronized object which is not circularized the elliptical instability is possible, it is then librational driven. Both forms of the elliptical instability will be considered in this study.

Worth mentioning is a study by Barker and Lithwick (2013a), they investigated the importance of the elliptical instability for tidal dissipation by three-dimensional hydrodynamic simulations. These were performed in a box with periodic boundary conditions. The base flow was chosen such that this box resembles a small patch in a tidally deformed fluid planet or star. They studied the nonlinear evolution of the elliptical instability and found that only for large ellipticities a state of sustained wave driving is present. But for the astrophysical relevant ellipticities strong columnar vortices form and this results in a significantly weaker dissipation compared to the case of sustained wave driving. Magnetic fields (Barker and Lithwick, 2014) or a strong stratification (Guimbard et al., 2010) can prevent the forming of these vortices. But also with sustained wave driving the inferred tidal dissipation accompanied by the elliptical instability is only enough to explain circularization and synchronization for binary stars and hot Jupiters with short periods (Barker and Lithwick, 2014); therefore, they conclude there might be an yet undetermined mechanism to explain circularization for wide orbital separations. They neglect the effects of a realistic geometry and the presence of turbulent convection.

The elliptical instability has been studied in different geometries, in an unbounded do- 
main (Bayly 1986; Landman and Saffman 1987; Waleffe 1990; Miyazaki and Fukumoto 1992; Miyazaki 1993; Miyazaki et al. 1995), elliptically deformed cylinder (Gledzer et al., 1975; Malkus, 1989; Eloy et al., 2003), deformed spheroid (Gledzer and Ponomarev, 1977; Kerswell, 1994; Cébron et al., 2010a), and spherical shells (Aldridge et al., 1997; SeyedMahmoud et al., 2000; Cébron et al., 2012). For the slightly elliptical deformed sphere, the geometry chosen in this study, also studies exist (Lacaze et al., 2004; Le Bars et al., 2010). The elliptical instability is influenced by stratification (Miyazaki and Fukumoto 1992; Miyazaki 1993; Guimbard et al. 2010), magnetic fields (Kerswell, 1994; Lacaze et al., 2006; Herreman et al., 2009), rotation of the elliptical perturbation (Craik, 1989; Gledzer and Ponomarev, 1992; Miyazaki, 1993; Miyazaki et al., 1995; Seyed-Mahmoud et al., 2000; Le Bars et al., 2010), and viscosity (Landman and Saffman, 1987; Kerswell, 1994; Lacaze et al., 2004; Le Bars et al., 2010). In this thesis rotation of the elliptical perturbation and viscosity are considered and additionally, contrary to all just mentioned studies here compressibility of the flow is taken into account. Nearly all other publications neglect this influence, an exception is the work by Cébron et al. (2013). They numerically simulated one particular set of parameters. Contrary to them, in this study, simplifying approximations were made such that it was possible to use semi-analytical methods. These enables us to obtain a broad overview of the stability characteristics instead of simulating one particular set of parameters as done by Cébron et al. (2013). They considered the nonlinear term in their calculation and therefore were able to calculate the amplitude of the instability at saturation, this work only focuses on the growth rate of the elliptical instability and for simplification the equations were linearized.

\subsection{Theoretical background}

\subsubsection{Mathematical formulation of the model}

We consider the equatorial tide raised on a central body by a tidal perturber. The frame in which the tidal perturber is at rest is the frame of reference. We use a Cartesian coordinate system $x, y, z$, with its origin at the center of the central body, its $z$-axis directed along the rotation axis of the reference system, and the $x$-axis is pointing towards the tidal perturber. This reference frame rotates at rate $\Omega_{\mathrm{p}}=\Omega_{\mathrm{p}} \hat{\mathbf{z}}$ (hats denote unit vectors) relative to inertial space. In this frame, the central body rotates about the $z$-axis with angular velocity $\frac{1}{2}\left(\frac{b}{a}+\frac{a}{b}\right) \Omega_{\mathrm{F}}$ within an ellipsoid with semi-major axes $a, b, c$ and the surface

$$
\left(\frac{x}{a}\right)^{2}+\left(\frac{y}{b}\right)^{2}+\left(\frac{z}{c}\right)^{2}=1,
$$


so that the motion of the central body within the chosen frame of reference is given by the velocity field $\mathbf{u}_{\mathbf{0}}$ :

$$
\mathbf{u}_{\mathbf{0}}=\Omega_{\mathrm{F}}\left(-\frac{a}{b} y \hat{\mathbf{x}}+\frac{b}{a} x \hat{\mathbf{y}}\right)
$$

In order to determine the stability of this flow, we start from the full Euler equations:

$$
\begin{gathered}
\partial_{t} \mathbf{u}+(\mathbf{u} \cdot \nabla) \mathbf{u}+2 \boldsymbol{\Omega}_{\mathrm{p}} \times \mathbf{u}=-\frac{1}{\rho} \nabla p+\nabla \Phi_{\text {self }}+\nabla \Phi_{\mathrm{t}} \\
\partial_{t} \rho+\nabla \cdot(\rho \mathbf{u})=0
\end{gathered}
$$

$\mathbf{u}$ stands for the velocity, $\rho$ the density, $p$ the pressure, and $\Phi_{\text {self }}$ for the potential terms created by the central body itself (gravitational and centrifugal), whereas $\Phi_{\mathrm{t}}$ is the perturbing tidal potential.

We assume $\mathbf{u}_{0}$ to be a stationary solution of the above equations for suitable density profiles and potentials $\rho_{0}$ and $\Phi_{0}$ :

$$
\begin{gathered}
\left(\mathbf{u}_{0} \cdot \nabla\right) \mathbf{u}_{0}+2 \boldsymbol{\Omega}_{\mathrm{p}} \times \mathbf{u}_{0}=-\frac{1}{\rho_{0}} \nabla p_{0}+\nabla \Phi_{0}+\nabla \Phi_{\mathrm{t}} \\
\nabla \cdot\left(\rho_{0} \mathbf{u}_{0}\right)=0 .
\end{gathered}
$$

In order to end with a tractable problem, we have to choose a density profile such that the eigenmode calculation below leads to a separable equation. This is achieved by setting

$$
\rho_{0}=\tilde{\rho}_{0}\left(1-\left(\frac{x}{a}\right)^{2}-\left(\frac{y}{b}\right)^{2}-\left(\frac{z}{c}\right)^{2}\right)^{\beta},
$$

for arbitrary prefactors $\tilde{\rho}_{0}$ and exponents $\beta$ (Wu, 2005a).

The question arises which $\beta$ one should use for the calculations. We must first determine which polytropic index $N_{\mathrm{p}}$ in the polytropic relation

$$
p(r)=K \rho^{1+1 / N_{\mathrm{p}}}(r)
$$

is appropriate for the central body. In this work $N_{\mathrm{p}}=3, N_{\mathrm{p}}=3 / 2$, and $N_{\mathrm{p}}=0$ are chosen for the calculations. $N_{\mathrm{p}}=3$ is suitable for stars which are well modeled by a relativistic completely degenerate electron gas, like relativistic white dwarfs. The same polytropic index also describes main sequence stars with $M \gtrsim M_{\odot}$, (Kippenhahn and Weigert, 1990). $N_{\mathrm{p}}=3 / 2$ is appropriate for objects which are well modeled by a 
non relativistic completely degenerate electron gas, like non-relativistic white dwarfs and brown dwarfs. And $N_{\mathrm{p}}=3 / 2$ is also relevant for main sequence stars with a mass below $M \sim 0.4 M_{\odot}$, these are fully convective stars (Kippenhahn and Weigert, 1990; Chabrier et al., 2009). For planets, the range of $N_{\mathrm{p}}$ is $0 \leq N_{\mathrm{p}} \lesssim 1.5$, depending on how massive they are (Horedt, 2004). In the polytropic case $\rho(r)$ can be obtained by solving the Lane-Emden equation. This equation can be derived through the usage of Eq. (2.9), the equation for hydrostatic equilibrium and the Poisson equation (Kippenhahn and Weigert, 1990). The appropriate $\beta$ were determined by simply fitting the power law Eq. (2.8) to the $\rho(r)$ for the polytropic profiles with $N_{\mathrm{p}}=3, N_{\mathrm{p}}=3 / 2$, and $N_{\mathrm{p}}=0$. The values for $\rho(r)$ in the polytropic case are taken from Horedt (1986).

It is easily verified that $\nabla \cdot\left(\rho_{0} \mathbf{u}_{0}\right)=0$ and $\nabla \times\left\{\left(\mathbf{u}_{0} \cdot \nabla\right) \mathbf{u}_{0}+2 \boldsymbol{\Omega}_{\mathrm{p}} \times \mathbf{u}_{0}\right\}=0$. The curl of the gradient terms in Eq. (2.6) is trivially zero. Therefore, after taking the curl of Eq. (2.6) only the term $\nabla \times\left\{\frac{1}{\rho_{0}} \nabla p_{0}\right\}=\frac{1}{\rho_{0}^{2}} \nabla \rho_{0} \times \nabla p_{0}$ is left. This term is zero if the interior of the central object is in hydrostatic equilibrium, because then

$$
0=-\nabla p_{0}-\rho_{0} \nabla\left(\Phi_{0}+\Phi_{\mathrm{t}}\right)
$$

Therefore $\nabla p_{0}$ must be aligned with $\nabla\left(\Phi_{0}+\Phi_{\mathrm{t}}\right)$. Taking the curl of Eq. (2.10) and using $\nabla \times \nabla=0$ yields $0=\nabla \times \rho_{0} \nabla\left(\Phi_{0}+\Phi_{\mathrm{t}}\right)=\nabla \rho_{0} \times \nabla\left(\Phi_{0}+\Phi_{\mathrm{t}}\right)$, hence $\rho_{0}$ must be aligned with $p_{0} . \mathbf{u}_{0}$ and $\rho_{0}$ as given above are therefore solutions of the Euler equation for some perturbing potential, albeit not necessarily for the perturbing potential of a point mass at a finite distance. $\rho_{0}$ can be viewed as an approximation to the real density profile, or as the exact profile for a perturbing potential which approximates a real tidal potential.

After introducing quantities which are decomposed into a value for the basic state, indicated by an index zero and a perturbation, which is considered to be small, the linear stability of the ground state can be investigated. The quantities are $\rho=\rho_{0}+\rho^{\prime}, \mathbf{u}=\mathbf{u}_{0}+\mathbf{u}^{\prime}$, $p=p_{0}+p^{\prime}$ and $\Phi_{\text {self }}=\Phi_{0}+\Phi^{\prime}$. Henceforth we omit the primes at the perturbation quantities, because only the perturbation and the background variables (denoted by the zero subscript) appear. The linearized equations are:

$$
\begin{gathered}
\partial_{t} \mathbf{u}+\left(\mathbf{u}_{0} \cdot \nabla\right) \mathbf{u}+(\mathbf{u} \cdot \nabla) \mathbf{u}_{0}+2 \boldsymbol{\Omega}_{\mathrm{p}} \times \mathbf{u}=-\frac{1}{\rho_{0}} \nabla p+\frac{\rho}{\rho_{0}^{2}} \nabla p_{0}+\nabla \Phi \\
\partial_{t} \rho+\nabla \cdot\left(\rho \mathbf{u}_{0}+\rho_{0} \mathbf{u}\right)=0 .
\end{gathered}
$$

We restrict ourselves to the consideration of only convective unstable objects, as $\mathrm{Wu}$ (2005a). In the paper by Cébron et al. (2010b) it was shown that even in flows with a stable stratified region and a convective region the instability can grow over the whole fluid, this is interesting for stars with a convective region on top of a radiative region, 
as in our sun, or vice versa. Due to the restriction on convective unstable objects the Brunt-Väisälä frequency is zero and therefore

$$
\nabla \rho_{0}=\frac{\rho_{0}}{\iota p_{0}} \nabla p_{0}
$$

with the adiabatic index $\iota$, which is given by $\iota=\partial \ln p_{0} /\left.\partial \ln \rho_{0}\right|_{s}$ and is related to the speed of sound by $\iota=c_{\mathrm{S}}^{2} \rho / p$. Furthermore, we assume that the perturbation is adiabatic,

$$
\frac{\delta p}{p_{0}}=\iota \frac{\delta \rho}{\rho_{0}}
$$

Lagrangian and Eulerian perturbation are related by

$$
\delta Z=Z+\boldsymbol{\xi} \cdot \nabla\left(Z_{0}+Z\right)
$$

with the displacement vector $\boldsymbol{\xi}$ and $Z$ the Eulerian perturbation, together with Eqs. (2.13) and (2.14) it gives:

$$
p / p_{0}=\iota \rho / \rho_{0}
$$

The right hand side of the equation for conservation of momentum Eq. (2.11) then simplifies according to $\nabla\left(\frac{p}{\rho_{0}}\right)=-\frac{\rho}{\rho_{0}^{2}} \nabla p_{0}+\frac{1}{\rho_{0}} \nabla p$.

The continuity equation can be simplified by using the anelastic approximation, which we will adopt from here on. This approximation is applicable as long as the rotation rate of the central object is much slower than the characteristic frequency $\Omega_{*}=\sqrt{G m_{\mathrm{ce}} / R_{\mathrm{ce}}^{3}}$ of the central object, with $G$ the gravitational constant and $m_{\mathrm{ce}}$ and $R_{\mathrm{ce}}$, mass and radius of the central object, respectively. More about the validity of the anelastic approximation for inertial modes excited by tides can be found e.g. in Ivanov and Papaloizou (2010).

The equation for conservation of momentum for the now simplified linear stability problem reads

$$
\partial_{t} \mathbf{u}+\left(\mathbf{u}_{0} \cdot \nabla\right) \mathbf{u}+(\mathbf{u} \cdot \nabla) \mathbf{u}_{0}+2 \boldsymbol{\Omega}_{\mathrm{p}} \times \mathbf{u}=\nabla \psi
$$

and with use of the anelastic approximation the continuity equation reads

$$
\nabla \cdot\left(\rho_{0} \mathbf{u}\right)=0
$$

with $\psi=\frac{p}{\rho_{0}}+\Phi$. This equations will be solved subject to the boundary condition $\hat{\mathbf{n}} \cdot \mathbf{u}=0$ on the ellipsoidal surface (2.2). This boundary conditions enforces that the pressure perturbation at the surface has to be zero, as will be shown later. A zero pressure perturbation is expected at the surface of a planetary or stellar atmosphere, because there is vacuum outside the surface. 
We now follow a procedure similar to that used by Gledzer and Ponomarev (1992) to determine the growth rates of the elliptical instability. The dimensions are removed by rescaling the Cartesian coordinates with their respective semi-major axis and the time with $\Omega_{\mathrm{F}}$,

$$
\begin{aligned}
& x^{*}=\frac{x}{a}, \quad y^{*}=\frac{y}{b}, \quad z^{*}=\frac{z}{c}, \quad u^{*}=\frac{u}{\Omega_{\mathrm{F}} a}, \quad v^{*}=\frac{v}{\Omega_{\mathrm{F}} b}, \\
& w^{*}=\frac{w}{\Omega_{\mathrm{F}} c}, \quad t^{*}=\Omega_{\mathrm{F}} t, \quad R=\sqrt{\frac{a^{2}+b^{2}}{2}}, \quad \psi^{*}=\frac{\psi}{\Omega_{\mathrm{F}}^{2} R^{2}}, \\
& \rho^{*}=\frac{\rho}{\tilde{\rho}_{0}},
\end{aligned}
$$

where $u, v$ and $w$ are the $x, y$ and $z$ components of $\mathbf{u}$, respectively. In the following we use the simplification $c=R=\sqrt{\left(a^{2}+b^{2}\right) / 2}$. Below it becomes plausible that a change of $\mathrm{c}$ will not cause substantial changes. In the following the stars above the rescaled quantities will be omitted, Eq. (2.17) then becomes

$$
\begin{gathered}
\frac{\partial u}{\partial t}+x \frac{\partial u}{\partial y}-y \frac{\partial u}{\partial x}-v-2 v \Omega \frac{b}{a}=-\frac{1}{1+\epsilon} \frac{\partial \psi}{\partial x} \\
\frac{\partial v}{\partial t}+x \frac{\partial v}{\partial y}-y \frac{\partial v}{\partial x}+u+2 u \Omega \frac{a}{b}=-\frac{1}{1-\epsilon} \frac{\partial \psi}{\partial y} \\
\frac{\partial w}{\partial t}+x \frac{\partial w}{\partial y}-y \frac{\partial w}{\partial x}=-\frac{\partial \psi}{\partial z} \\
\nabla \cdot\left(\rho_{0} \mathbf{u}\right)=0
\end{gathered}
$$

with $\epsilon$ the ellipticity of the boundaries in the $x, y$-plane $\epsilon=\left(a^{2}-b^{2}\right) /\left(a^{2}+b^{2}\right)$ and $\Omega=\Omega_{\mathrm{p}} / \Omega_{\mathrm{F}}$. And the density profile Eq. (2.8) becomes

$$
\rho_{0}=\left(1-x^{2}-y^{2}-z^{2}\right)^{\beta}
$$

In this nondimensionalized variables initial elliptical streamlines correspond to circular streamlines.

The next step is a switch from Cartesian coordinates to cylindrical coordinates $(s, z, \varphi)$. In cylindrical coordinates, the above system of equations is written as

$$
\mathbf{M}\left(\frac{\partial \mathbf{u}}{\partial t}+\mathbf{H u}\right)+2 \Omega\left(1-\epsilon^{2}\right)^{1 / 2} \boldsymbol{\Lambda} \mathbf{u}=-\nabla \psi, \quad \nabla \cdot\left(\rho_{0} \mathbf{u}\right)=0
$$

with

$$
\begin{aligned}
& \mathbf{M}=\mathbf{I}+\epsilon \mathbf{T}, \quad \mathbf{H}=\mathbf{I} \frac{\partial}{\partial \varphi}+2 \boldsymbol{\Lambda} \\
& \mathbf{T}=\left(\begin{array}{ccc}
\cos (2 \varphi) & -\sin (2 \varphi) & 0 \\
-\sin (2 \varphi) & -\cos (2 \varphi) & 0 \\
0 & 0 & 0
\end{array}\right), \quad \boldsymbol{\Lambda}=\left(\begin{array}{ccc}
0 & -1 & 0 \\
1 & 0 & 0 \\
0 & 0 & 0
\end{array}\right)
\end{aligned}
$$


where $\mathbf{I}$ is the identity matrix. If the calculations were not restricted to a certain ellipsoidal shape, with $c=R$, additional terms would appear in the equation $\mathbf{M}=\mathbf{I}+\epsilon \mathbf{T}$ (SeyedMahmoud et al., 2000). We use the ansatz

$$
\begin{aligned}
& \mathbf{u}=\sum_{j} C_{j} \mathbf{u}_{\mathbf{j}} e^{\mathrm{i} f t}, \\
& \psi=\sum_{j} C_{j} \psi_{j} e^{\mathrm{i} f t},
\end{aligned}
$$

for the solution of Eq. (2.23), where the $\mathbf{u}_{\mathbf{j}}$ are solutions of the unperturbed eigenvalue problem $(\epsilon=0)$

$$
\begin{array}{r}
\mathbf{H} \mathbf{u}_{\mathbf{j}}+\nabla \psi_{j}+2 \boldsymbol{\Lambda} \mathbf{u}_{\mathbf{j}} \Omega=-\mathrm{i} \omega_{j} \mathbf{u}_{\mathbf{j}}, \quad \nabla \cdot\left(\rho_{0} \mathbf{u}_{\mathbf{j}}\right)=0, \\
\hat{\mathbf{n}} \cdot \mathbf{u}_{\mathbf{j}}=0 \quad \text { on the unit sphere }
\end{array}
$$

This solution method is called Galerkin method. Eq. (2.25) is the equation for inertial modes in a sphere. $j$ is a proxy for the indices $\left(n_{j}, m_{j}, k_{j}\right)$ which characterize an inertial mode in a sphere (Greenspan, 1968). If necessary the variables $n, m$, and $k$ will be indexed to distinguish different modes, otherwise they appear without an index. $n$ is the spatial degree (on the surface of the sphere, the pressure distribution is given by the spherical harmonic $\left.Y_{n}^{k}\right), k$ the azimuthal wavenumber, and $m$ a number which has a finite range, as a third number one can also use the frequency of the mode $\omega$ instead of $m$. For the incompressible case it is possible to obtain an analytical solution of the equations (Zhang et al., 2001). Within the anelastic approximation and the restriction on density profiles of the form

$$
\rho_{0}=\left(1-s^{2}-z^{2}\right)^{\beta}
$$

the equations are separable.

We now show that vacuum outside the surface entails that the pressure perturbation at the surface has to be zero, as in $\mathrm{Wu}(2005 \mathrm{a})$. We use spherical coordinates $(r, \theta, \varphi)$ in this abstract. In these spherical coordinates the above density profile becomes $\rho_{0}=\left(1-r^{2}\right)^{\beta}$. Furthermore we use the continuity equation in the form

$$
\nabla \cdot(\rho \boldsymbol{\xi})=\nabla \cdot \boldsymbol{\xi}-\frac{\mathbf{e}_{r} \cdot \boldsymbol{\xi}}{H}=0
$$

with the density scale height $H \equiv-d r / d \ln \rho=c_{\mathrm{s}}^{2} / g$ with $g$ the local gravitational acceleration. The zero pressure perturbations condition written in the convenient form $\delta p / \rho_{0}=0$ (Unno et al., 1989) gives

$$
\delta p / \rho_{0}=\iota \frac{\delta \rho}{\rho_{0}} \frac{p_{0}}{\rho_{0}}=-\iota(\nabla \cdot \boldsymbol{\xi}) \frac{p_{0}}{\rho_{0}}=-g \xi_{r}
$$


because $u_{r}=i \omega \xi_{r}$ this relates to $\hat{\mathbf{n}} \cdot \mathbf{u}=0$ at the surface.

Repeating the steps of $\mathrm{Wu}$ (2005a) (see Appendix A), one arrives at an eigenvalue problem for $\psi$ which is written as a product in the form $\psi\left(\bar{x}_{1}, \bar{x}_{2}, \bar{\varphi}, t\right)=\psi_{1}\left(\bar{x}_{1}\right) \psi_{2}\left(\bar{x}_{2}\right) J(\bar{\varphi}, t)$. $\left(\bar{x}_{1}, \bar{x}_{2}, \bar{\varphi}\right)$ are the ellipsoidal coordinates used in Wu (2005a) and introduced by Bryan (1889). It is $\bar{\varphi}=\varphi+t$. In this chapter an overline about a coordinates indicates that this coordinate is chosen with respect to a reference frame rotating with $\Omega_{\mathrm{F}}+\Omega_{\mathrm{p}}$. Furthermore, one must be careful to distinguish the index which characterizes the different inertial modes from the index used in Eqs. (2.29) and (2.30) which stands for the two coordinates $\bar{x}_{1}$ and $\bar{x}_{2}$. In this paragraph we omit the index which characterizes the different inertial modes. The range of the three coordinates is $\bar{x}_{1} \in[\zeta, 1], \bar{x}_{2} \in[-\zeta, \zeta]$, with $\zeta=-(\omega+k) /(2(1+\Omega))$ and $\bar{\varphi}$ is the azimuthal angle such that $\bar{\varphi} \in[0,2 \pi] . \quad-\zeta$ is half the frequency of the inertial mode with respect to the frame rotating with $\Omega_{\mathrm{p}}+\Omega_{\mathrm{F}}$ and $\omega$ is the frequency with respect to the frame rotating with $\Omega_{\mathrm{p}}$. All variables $X$ (pressure and velocity components) depend on time $t$ and on the azimuthal angle $\varphi$, respectively $\bar{\varphi}$, through the relation $X \propto J(\bar{\varphi}, t)=\exp [\mathrm{i}(k \bar{\varphi}-2 \zeta(\Omega+1) t]=\exp [\mathrm{i}(k \varphi+\omega t]$. We adopt the convention that $\zeta \geq 0$ with $k>0$ representing a prograde mode and $k<0$ a retrograde mode. Modes with denotation $(k, \zeta)$ and $(-k,-\zeta)$ are physically the same modes so that one can restrict either $\zeta$ or $k$ to positive numbers and avoid redundancy. It is numerically more accurate to solve for the $g_{i}$ defined by $g_{i}=\psi_{i} /\left[\left(1-\bar{x}_{i}^{2}\right)^{|k| / 2}(\omega+k)^{2}\right], i=1,2$. The eigenvalue problem for the eigenvalue $l_{\mathrm{e}}$ and the eigenfrequency $\zeta$ reads

$$
\begin{array}{r}
\left(1-\bar{x}_{i}^{2}\right) \frac{\mathrm{d}^{2} g_{i}}{\mathrm{~d} \bar{x}_{i}^{2}}-2 \bar{x}_{i}(|k|+1) \frac{\mathrm{d} g_{i}}{\mathrm{~d} \bar{x}_{i}}+\frac{2 \beta \bar{x}_{i}\left(1-\bar{x}_{i}^{2}\right)}{\bar{x}_{i}^{2}-\zeta^{2}} \frac{\mathrm{d} g_{i}}{\mathrm{~d} \bar{x}_{i}} \\
+\left[o^{2}-\frac{2 \beta|k| \bar{x}_{i}^{2}}{\bar{x}_{i}^{2}-\zeta^{2}}+\frac{2 \beta k \zeta}{\bar{x}_{i}^{2}-\zeta^{2}}\right] g_{i}=0,
\end{array}
$$

with the boundary conditions

$$
\begin{aligned}
\left.\frac{\mathrm{d} g_{1}}{\mathrm{~d} \bar{x}_{1}}\right|_{\bar{x}_{1}=1} & =\left.\frac{o^{2}+2 \beta\left[(k \zeta-|k|) /\left(1-\zeta^{2}\right)\right]}{2(|k|+1)} g_{1}\right|_{\bar{x}_{1}=1}, \\
\left.\frac{\mathrm{d} g_{1}}{\mathrm{~d} \bar{x}_{1}}\right|_{\bar{x}_{1}=\zeta} & =-\left.\frac{(k-|k| \zeta)}{1-\zeta^{2}} g_{1}\right|_{\bar{x}_{1}=\zeta}, \\
\left.\frac{\mathrm{d} g_{2}}{\mathrm{~d} \bar{x}_{2}}\right|_{\left|\bar{x}_{2}\right|=\zeta} & =-\left.\operatorname{sgn}\left(\bar{x}_{2}\right) \frac{(k-|k| \zeta)}{1-\zeta^{2}} g_{2}\right|_{\left|\bar{x}_{2}\right|=\zeta},
\end{aligned}
$$

and $o^{2}=l_{\mathrm{e}}\left(l_{\mathrm{e}}+1\right)-|k|(|k|+1)$. In general, both $\zeta$ and $l_{\mathrm{e}}$ have to be determined numerically, but for $\beta=0, l_{\mathrm{e}}$ is simply given by $l_{\mathrm{e}}=n$. 
For the following calculation the scalarproduct is defined as

$$
\left(\mathbf{u}_{\mathbf{j}}, \mathbf{u}_{\mathbf{i}}\right)=\int_{V} \mathbf{u}_{\mathbf{j}} \mathbf{u}_{\mathbf{i}}^{*} \rho_{0} r d r d \varphi d z
$$

The eigenvalues calculated in the anelastic approximation are orthogonal with this scalar product. The proof follows the proceeding in Greenspan (1968), see Appendix B.

We substitute (2.24) in (2.23) and multiply this equation by $\rho_{0} \mathbf{u}_{\mathbf{j}}$. This yields the system

$$
\begin{array}{r}
\left(f-\omega_{j}\right) C_{j} N_{j}^{2}+\epsilon\left(f+k_{j}\right) \sum_{i} V_{j, i} C_{i}=0, \\
N_{j}^{2}=\left(\mathbf{u}_{\mathbf{j}}, \mathbf{u}_{\mathbf{j}}\right), \quad V_{i, j}=\left(\mathbf{T} \mathbf{u}_{\mathbf{j}}, \mathbf{u}_{\mathbf{i}}\right) .
\end{array}
$$

We assume the $\mathbf{u}_{\mathbf{j}}$ to be normalized such that $N_{j}^{2}=1$. For incompressible fluids it was proven analytically that $V_{i, j} \neq 0$ only for $n_{i}=n_{j}$ and $k_{i}=k_{j} \pm 2$ by Kerswell (1993). That the latter condition also holds in the compressible case is easy to see, because the dependence of the variables on the azimuthal angle is given by $\exp (i k \varphi)$. The first condition is less obvious. It was proven analytically by Kerswell (1993) for incompressible fluids. This proof can not simply be adapted to the compressible case because no analytical expressions exist for the modes in this case. Therefore we calculated the $V_{i, j}$ numerically, for all mode combinations with indices up to $n=20$ and $|k|=10$. In all cases, the results appeared to converge to zero as the spatial resolution used in the integration was improved. Due to the foregoing it can be deduced that the elliptical instability is the result of the interaction of modes with azimuthal wavenumber $k$ and $k+2$ and the spatial degree $n$ of the interacting modes must be the same. For $\epsilon \ll 1$ instability is possible only for modes with frequencies close to each other. With $\omega_{i}$ and $\omega_{j}$ such that $\omega_{i} \approx \omega_{j}$, and $k_{i}=k_{j}+2$ or $k_{i}=k_{j}-2$ at first order of perturbation theory Eq. (2.32) may be reduced to

$$
\begin{aligned}
\left(f-\omega_{i}\right) C_{i} N_{i}^{2}+\epsilon\left(f+k_{i}\right) V_{i, j} C_{j} & =0, \\
\epsilon\left(f+k_{j}\right) V_{j, i} C_{i}+\left(f-\omega_{j}\right) C_{j} N_{j}^{2} & =0,
\end{aligned}
$$

this leads to the quadratic equation

$$
\begin{array}{r}
\left(1-W^{2}\right) f^{2}-\left[\omega_{i}+\omega_{j}+W^{2}\left(k_{i}+k_{j}\right)\right] f+\left(\omega_{i} \omega_{j}-W^{2} k_{j} k_{i}\right)=0, \\
W^{2}=\epsilon^{2} \frac{V_{i, j} V_{j, i}}{N_{i}^{2} N_{j}^{2}} .
\end{array}
$$


The result is a growth rate $\sigma_{\text {inv }}$ and frequency $\omega_{\mathrm{e}}\left(f=\omega_{\mathrm{e}}+i \sigma_{\mathrm{inv}}\right)$

$$
\begin{aligned}
\sigma_{\mathrm{inv}}^{2} & =-\frac{\left(W^{2}\left(k_{i}-k_{j}\right)+\Delta \omega\right)^{2}+4 W^{2}\left(\omega_{i}+k_{i}\right)\left(\omega_{j}+k_{j}\right)}{4\left(1-W^{2}\right)^{2}}, \\
\omega_{\mathrm{e}} & =\frac{\omega_{i}+\omega_{j}+W^{2}\left(k_{i}+k_{j}\right)}{2\left(1-W^{2}\right)}
\end{aligned}
$$

with $\Delta \omega=\omega_{i}-\omega_{j}$. Eqs. (2.35) and (2.36) are the same as Eq. (3.5) of Gledzer and Ponomarev (1992). It follows from this equation that instability is possible only if $|\Delta \omega| \leq$ $O(\epsilon)$. Modes in resonance will be written as $(k, k+2)$ and the spatial degree $n$ will be stated separately. To consider viscosity in the calculations a damping rate is added to $\sigma_{\text {inv }}$. The damping rate depends on the applied boundary condition. For free slip boundary conditions the approximation is

$$
\sigma_{\mathrm{fs}}=\sigma_{\mathrm{inv}}-h_{1} n(n+1) E k
$$

and for no slip boundary conditions

$$
\sigma_{\mathrm{ns}}=\sigma_{\mathrm{inv}}-h_{2}|1+\Omega| \sqrt{E k}-h_{1} n(n+1) E k .
$$

$h_{1}$ and $h_{2}$ are constants of order 1 which in this study are always chosen as $h_{1}=h_{2}=1$ and $E k$ is the Ekman number. This is a heuristic approach. The term $\propto E k$, which appears in both equations corresponds to dissipation in the bulk. $n(n+1)$ appears in this term, because the velocity field of an inertial mode contains on the surface only the spherical harmonic $Y_{n}^{k}$. Assuming this is a reasonable approximation to the inertial mode at any radius $r$, and using

$$
\nabla^{2} Y_{n}^{k}(\Theta, \varphi)=-\frac{(n+1) n}{r^{2}} Y_{n}^{k}
$$

one justifies the formula for $\sigma_{\mathrm{fs}}$ (Lorenzani, 2001). The term $\propto \sqrt{E} k$ which appears only in the equation with no slip boundary conditions is due to friction inside Ekman layers at the boundaries. This term is usually computed in the frame of reference in which the boundaries are at rest and expressed in multiples of the rotation rate of that frame, yielding decay rates of inertial modes of the form $h_{2} \sqrt{E k}$. Transformed to the frame of reference and the unit of time used in Eq. (2.19), the decay rate is $g_{1}|1+\Omega| \sqrt{E k}$ with $E k=\nu /\left(\left|\Omega_{F}+\Omega_{P}\right| R^{2}\right)$ and $\nu$ the viscosity. 


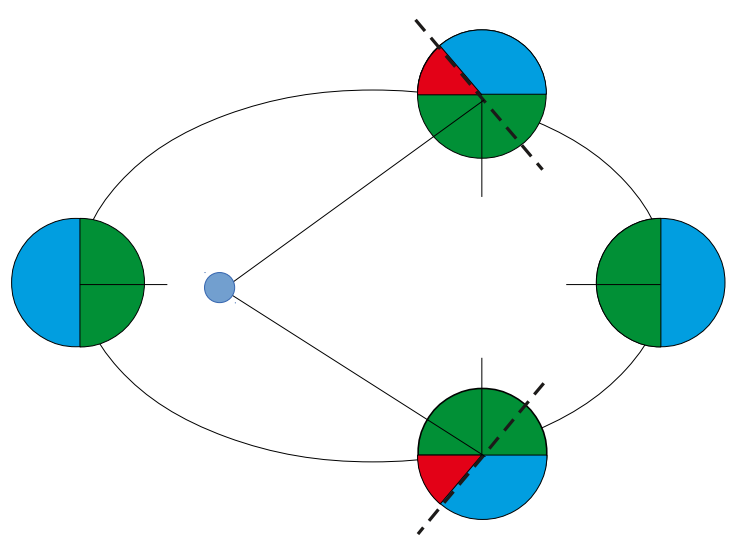

Figure 2.1.: The elliptic orbit is the reason for the libration of the central object (big sphere) on its orbit around the tidal perturber (small sphere). The orbit is synchronized but not circularized. One face of the central object is always pointing at the empty focus of the ellipse, while the tidal bulge (which is not plotted here) always points to the tidal pertuber (the occupied focus) (Murray and Dermott, 1999). Therefore the red parts of the central objects can be seen from the tidal perturber, caused by the libration. On a circular orbit only the green parts would be visible from the tidal perturber (see figure in zum.de/Faecher/Materialien/gebhardt/astronomie/libration.html).

\subsubsection{Librational driven elliptical instability}

We consider now the case that the objects spin is already synchronized but its orbit is not circularized. As already written in the introduction to this chapter the librational driven elliptical instability appears in fluid central objects which are not circularized on their orbit around their tidal perturber. That means the orbit is eccentric, and for an eccentric orbit the central objects orbital angular velocity equals its rotational angular velocity only on average, as illustrated in Fig. 2.1. The proceeding here is similar to that in Kerswell and Malkus (1998). We take the $x-y$ plane as the orbital plane. Time is nondimensionalized such that the basic spin of the central object has an angular velocity of $\hat{\mathbf{z}}$. The tidal bulge has then angular velocity $\Omega_{\mathrm{b}}(t) \hat{\mathbf{z}}$, with

$$
\Omega_{\mathrm{b}}(t)=1-\varpi_{1} \sum_{c_{1}=1}^{\infty} a_{c_{1}} \cos c_{1} t
$$

since $\Omega_{\mathrm{b}}(t)$ is $2 \pi$ periodic. In the following we assume that $\left|\varpi_{1}\right| \ll 1$. In the frame of the tidal bulge the background flow of the fluid is elliptical

$$
\mathbf{u}_{0}=\left(1-\Omega_{\mathrm{b}}(t)\right)(-(1+\epsilon) y \hat{\mathbf{x}}+(1-\epsilon) x \hat{\mathbf{y}}) .
$$


We consider the Euler equation and determine the stability of the flow,

$$
\begin{gathered}
\frac{\partial \mathbf{u}}{\partial t}+2 \Omega_{\mathrm{b}}(t) \hat{\mathbf{z}} \times \mathbf{u}+\frac{\mathrm{d} \Omega_{\mathrm{b}}}{\mathrm{d} t} \hat{\mathbf{z}} \times \mathbf{r}+\mathbf{u} \cdot \nabla \mathbf{u}+\nabla \psi=0, \\
\nabla \cdot\left(\rho_{0} \mathbf{u}\right)=0,
\end{gathered}
$$

whereby the anelastic approximation is used and $\psi$ has the same meaning as $\psi$ in the preceding section. The linear stability problem for the perturbation flow writes

$$
\begin{gathered}
\partial_{t} \mathbf{u}+\left(\mathbf{u}_{0} \cdot \nabla\right) \mathbf{u}+(\mathbf{u} \cdot \nabla) \mathbf{u}_{0}+2 \Omega_{\mathrm{b}}(t) \hat{\mathbf{z}} \times \mathbf{u}=\nabla \psi \\
\nabla \cdot\left(\rho_{0} \mathbf{u}\right)=0 .
\end{gathered}
$$

Multiplying (2.44) by $\rho_{0} \mathbf{u}$ yields

$$
\frac{\mathrm{d}\left(\rho_{0} \mathbf{u}^{2} / 2\right)}{\mathrm{d} t}+\rho_{0} \mathbf{u} \cdot\left(\mathbf{u} \cdot \nabla \mathbf{u}_{0}\right)=-\rho_{0} \mathbf{u} \cdot \nabla \psi
$$

Integrating about the volume and dividing by $<\rho_{0} \mathbf{u}^{2}>$, where $<>=\int d V$, gives

$$
\sigma(t)=\frac{1}{2} \frac{\mathrm{d}}{\mathrm{d} t} \ln <\frac{1}{2} \rho_{0} \mathbf{u}^{2}>=-\frac{<\rho_{0} \mathbf{u} \cdot \nabla \mathbf{u}_{0} \cdot \mathbf{u}>}{<\rho_{0} \mathbf{u}^{2}>}=\frac{\epsilon\left(1-\Omega_{\mathrm{b}}(t)\right)<2 \rho_{0} u_{x} u_{y}>}{<\rho_{0} \mathbf{u}^{2}>} .
$$

For the solution we make an ansatz consisting of a superposition of two inertial waves, with each satisfying the equation for the undeformed not librating sphere $\left(\varpi_{1}=0\right.$ and $\epsilon=0$ ). It is $<2 \rho_{0} u_{x i} u_{y j}>=V_{i, j}$ (see Eq. (2.32)), as one can easily see by transforming from cartesian to cylindrical coordinates. Therefore, from Eq. (2.47) it can be deduced that a resonance with $q_{i}-q_{j}= \pm c_{1}\left(c_{1} \in \mathbb{Z}\right), k_{i}-k_{j}= \pm 2$ and $n_{i}=n_{j}$ between two inertial modes (with frequencies $q_{i}$ and $q_{j}$ ) is necessary to obtain a non-vanishing mean growth rate. In the following we consider the case $c_{1}=1$, because if hypothetically the central objects orbital and spin angular momenta are constant, it is $a_{1}=2$ and $a_{c_{1}}=O\left(\varpi_{1}\right)$ for $c_{1}>1$ (see e.g. Colwell 1992). This indicates that the $c_{1}=1$ resonance is of most importance. For the not librational driven elliptical instability and perfect resonance it needs to be $\Delta \omega=0$, with $\omega_{i}=(1+\Omega) q_{i}-k_{i}$ for all $i$. The frequencies $q_{i}$ and $q_{j}$ have then to obey

$$
q_{i}-q_{j}=\frac{k_{i}-k_{j}}{1+\Omega}
$$

We consider now the case $\Omega=1$, then the resonance conditions for both kinds of elliptical instabilities are the same. Correspondingly we can deduce the nondimensionalized growth rate of the librational driven elliptic instability $\sigma /\left(\epsilon \varpi_{l}\right)$ equals the nondimensionalized growth rate of the not librational driven elliptic instability $\sigma / \epsilon$ for the case $\Omega=1$, because then the same modes interact with each other. 
Also for the librational induced elliptical instability imperfect resonances are possible. Then a frequency detuning as in Cébron et al. (2014) has to be considered.

\subsection{Numerical implementation}

We used numerical methods to calculate the growth rates. We need the frequencies and velocity fields for the modes to calculate the growth rates according to Eq. (2.35). First the frequencies for the inertial modes with $\beta=0$ were calculated by solving Eq. (29) from $\mathrm{Wu}(2005 \mathrm{a})$

$$
\left.\frac{\mathrm{d} P_{n}^{k}\left(\bar{x}_{1}\right)}{\mathrm{d} \bar{x}_{1}}\right|_{\bar{x}_{1}=\zeta}=-\left.\frac{k}{1-\zeta^{2}} P_{n}^{k}\left(\bar{x}_{1}\right)\right|_{\bar{x}_{1}=\zeta}
$$

by bisection. These eigenfrequencies together with the corresponding eigenvalues $l_{e}$, with $l_{e}=n$ for the incompressible case, are used as starting values for a shooting method to solve Eq. (2.29). In Wu (2005a) it is written "the number of eigenmodes remains conserved when $\beta$ varies, with a close one-to-one correspondence between modes in different density profiles". Therefore we obtained the frequencies by incrementing $\beta$ in steps of 0.1 and use the eigenvalues of the foregoing $\beta$ as an initial point for the shooting method. A Runge Kutta Cash-Karp method of fifth order with an adaptive stepsize control was implemented to integrate the ODE. Because the endpoints of the ODE for one variable are both singular points, the method of shooting to a fitting point was used (Press et al., 1992). To calculate the integrals for Eq. (2.35) only the modes which satisfy the resonance condition have to be considered. A simple calculations shows that instability is only possible if $|\Delta \omega|<4 \epsilon+2 \epsilon^{2}$ (Appendix C).

We solved the integrals $V_{i, j}$ by Gaussian quadrature. This calculation can not be performed in the ellipsoidal coordinate space $\left(\bar{x}_{1}, \bar{x}_{2}, \bar{\varphi}\right)$ because in these coordinates the limits of integration depend on the eigenfrequencies and in general the two wave functions have two different eigenfrequencies. Therefore the integration was performed in $(\bar{s}, \bar{z}, \bar{\varphi})$ space. The overline indicates that these coordinates are chosen with respect to a reference frame rotating with $\Omega_{\mathrm{F}}+\Omega_{\mathrm{p}}$. We obtained the scalars $\psi_{1}$ and $\psi_{2}$ at several points $\bar{x}_{1}$ and $\bar{x}_{2}$ by the numerical integration of the ODE (2.29). The final integrations were performed using Gaussian quadrature with Gauss-Legendre abscissas and weights with a resolution of 50 points in both $\bar{s}$ and $\bar{z}$ directions. The integration in $\bar{\varphi}$ direction can be performed analytically. We know $\bar{x}_{1}(\bar{s}, \bar{z})$ and $\bar{x}_{2}(\bar{s}, \bar{z})$ and obtain $\psi_{1}\left(\bar{x}_{1}(\bar{s}, \bar{z}), \bar{x}_{2}(\bar{s}, \bar{z})\right) \psi_{2}\left(\bar{x}_{1}(\bar{s}, \bar{z}), \bar{x}_{2}(\bar{s}, \bar{z})\right)$. The scalars $\psi_{1}$ and $\psi_{2}$ were obtained at some points $\bar{x}_{1}$ and $\bar{x}_{2}$ which in general do not coincide with the points we need for the gaussian quadrature. Therfore we need the scalars $\psi_{1}, \psi_{2}$ at arbitrary points $\bar{x}_{1}, \bar{x}_{2}$. This was accomplished by a cubic-spline interpolation. 


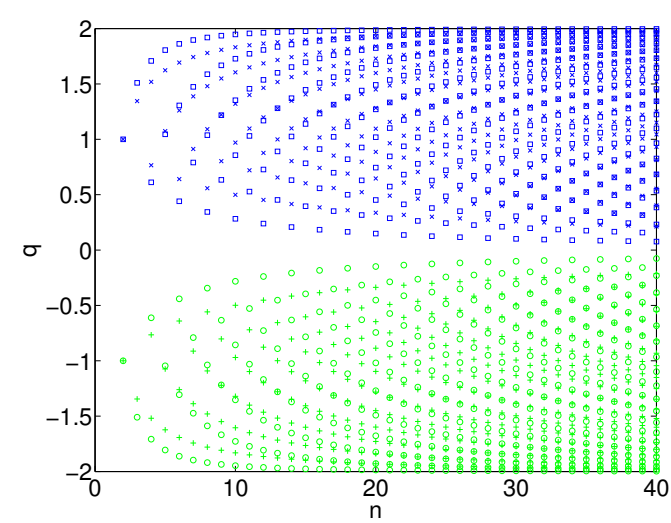

(a) Modes with $k q>0$

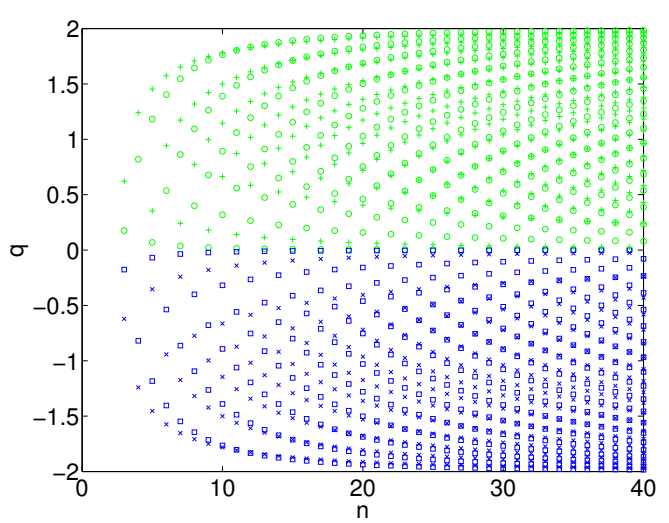

(b) Modes with $k q<0$

Figure 2.2.: Frequencies of inertial modes in the co-rotating frame, $q$, for $\beta=0$ (cross) and $\beta=18$ (circle) as a function of the spatial degree $n$. Only the modes with $k=-1$ (green) and $k=1$ (blue) are shown. Credit: Clausen \& Tilgner, A\&A, 562, A25, 2014, reproduced with permission CESO.

\subsection{Results}

First we consider inviscid flows and include viscous effects at the end of this section. In Fig. 2.2 the dispersion relation for $k=-1$ and $k=1$ for incompressible fluids and for $\beta=18$ is shown. There is a noteworthy distinction between the cases $k q>0$ and $k q<0$ in that the modes with the frequency $q$ with the smallest absolute value at any given $n$ always occurs for $k q<0$. These low frequency modes will be important later. The mode frequencies change continuously with $\beta$ so that individual modes can be tracked as a function of $\beta$. It depends on $\beta$ if a chosen triad leads to instability or not, this can be seen in Fig. 2.3(a) and Fig. 2.4(a). The structure of the mode as well as the frequency are changed if $\beta$ is changed, and hence also the integrals in $V_{i, j}$ and $\Delta \omega$ in Eq. (2.35). The impact of the detuning can be seen if one additionally considers Fig. 2.3(b) and Fig. 2.4(b). The fact that positive growth rates are only possible for $|\Delta \omega| \leq O(\epsilon)$ is displayed here. An exception is the mode with $|k|=1$ and $n=2$ because it corresponds to the purely toroidal motion in the spin-over mode. Toroidal modes have no radial velocity component; therefore, with a radially dependent background density profile $\rho_{0}$, as in this study, $\rho_{0}$ drops from the continuity equation $\nabla \cdot\left(\rho_{0} \mathbf{u}\right)=0$. As a consequence eigenvalue problem and stability problem are independent of $\rho_{0}$, and therefore $\beta$. This fact can be seen in Fig. 2.3 and Fig. 2.4 for the triad with $(-1,1)$ and $n=2$. But this triad is the only one where the modes are in perfect resonance with $\Delta \omega=0$.

The tidal flow is unstable if any two inertial modes form a triad with positive growth rate. 


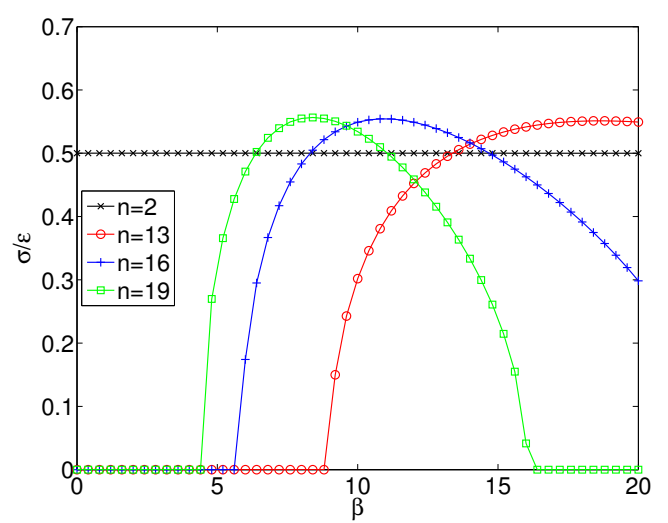

(a)

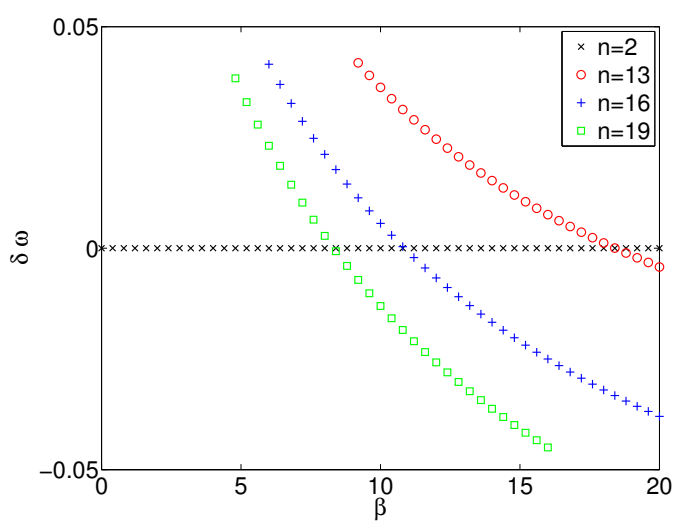

(b)

Figure 2.3.: Growth rates of modes $(-1,1)$ for various $n$ as a function of the compressibility parameter $\beta$ for $\epsilon=0.04, \Omega_{\mathrm{p}}=0$, and $E k=0$ (a). The corresponding $\Delta \omega$ (b). Credit: Clausen \& Tilgner, A\&A, 562, A25, 2014, reproduced with permission @ESO.

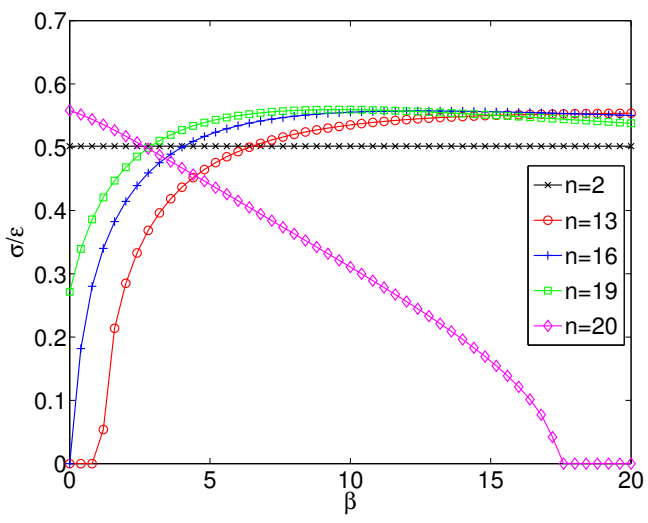

(a)

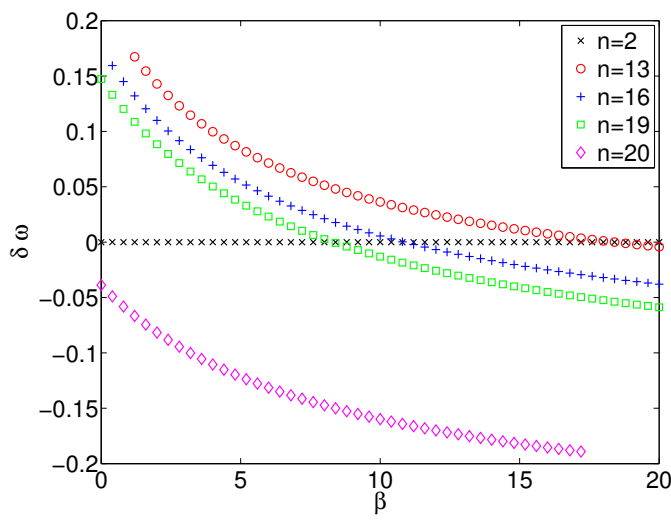

(b)

Figure 2.4.: Same as Fig. 2.3 but for $\epsilon=0.16$. Credit: Clausen \& Tilgner, A\&A, 562, A25, 2014, reproduced with permission @ESO. 


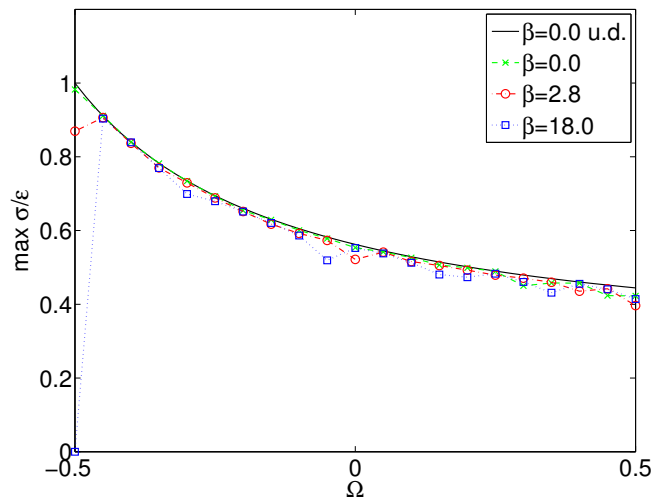

(a) $\epsilon=1 \times 10^{-2}, E k=0$

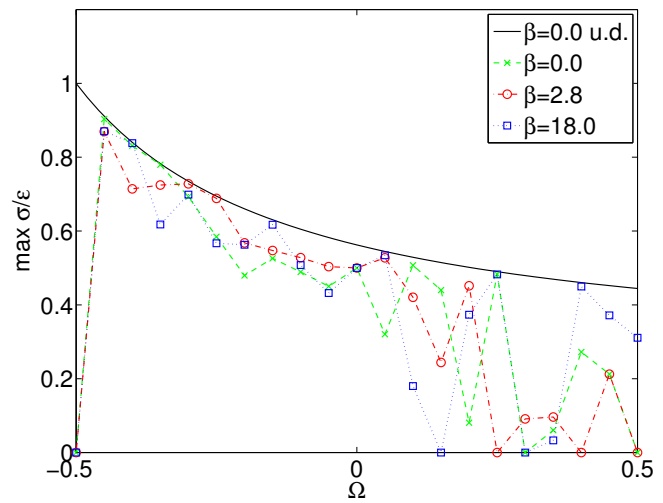

(b) $\epsilon=1 \times 10^{-3}, E k=0$

Figure 2.5.: Maximum growth rates as a function of $\Omega$ for $\mathrm{Ek}=0$. For this figure we take into account all modes with $|k| \leq 10$ and $n \leq 40$. The black line in this figure is the growth rate given by Eq. (2.50). Credit: Clausen \& Tilgner, A\&A, 562, A25, 2014, reproduced with permission $\odot$ ESO.

The main concern is if the flow as whole is stable or not. Therefore one has to consider not the growth rate of single modes, but since there exist an infinite number of modes, a restriction has to be made. Figures 2.5(a) and 2.5(b) is the result of a scan through all possible triads out of modes with $|k| \leq 10$ and $n \leq 40$. In this figures the maximum growth rate of this scan for a certain $\epsilon$ as a function of $\Omega$ for different $\beta$ is shown. Resonances are impossible in the interval $-3 / 2<\Omega<-1 / 2$ (Craik, 1989), therefore we choose a part of the complementary interval by way of example. The various curves appear to have a common envelope $\sigma_{\text {ud }}$ given by (Miyazaki et al., 1995)

$$
\sigma_{\mathrm{ud}}=\frac{(3+2 \Omega)^{2}}{16(1+\Omega)^{2}} \epsilon
$$

Miyazaki et al. (1995) calculated this inviscid growth rate for an incompressible, infinitely extended fluid. Also this curve is plotted in Fig. 2.5 (the black line). There are deviations between Eq. (2.50) and the computed maximum growth rates. But it seems that they get smaller if a bigger $\epsilon$ is chosen. This makes sense because at a certain $\epsilon$ only the modes with $|\Delta \omega| \leq O(\epsilon)$, required by Eq. (2.35), are potential candidates for a resonance, the smaller $\epsilon$ the less modes fulfill this criterion. Therefore for a smaller $\epsilon$ more modes are needed to minimize the deviations between Eq. (2.50) and the computed maximum growth rate, because as can be seen in Fig. 2.2 with higher spatial wavenumber $n$ the frequencies get denser. It is plausible to assume that the deviations will entirely disappear if all inertial modes are taken into consideration. From the foregoing it can be deduced that $\beta$ determines which modes are unstable but the stability limit does not depend on $\beta$, at least in the inviscid case. 


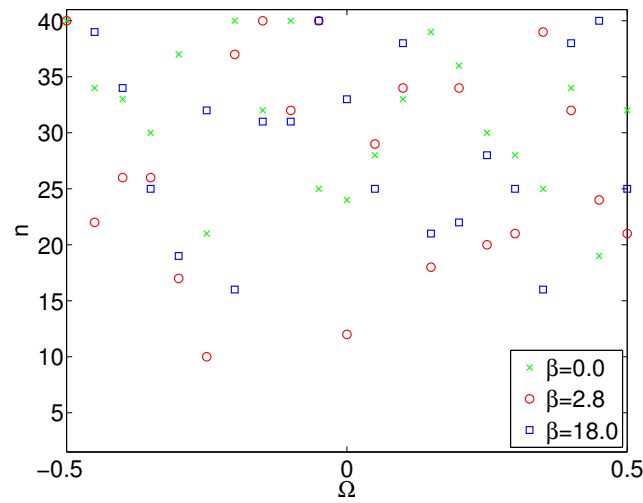

(a) $\epsilon=1 \times 10^{-2}, E k=0$

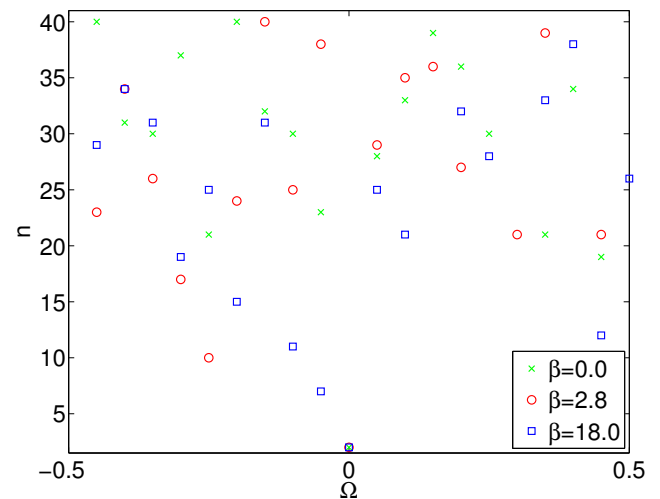

(b) $\epsilon=1 \times 10^{-3}, E k=0$

Figure 2.6.: The corresponding values of the spatial degree $n=n_{i}=n_{j}$ for the growth rates in Fig. 2.5.

In the following it will be determined how the necessary number of modes for an unstable flow changes, if one changes $\epsilon$, assuming that the frequencies are statistically uniformly distributed. The number of modes depend on the spatial degree $n$ and therefore also a relation between the minimum $n$ to obtain an unstable flow and $\epsilon$ will be derived. A resonance can only occur if two modes $i$ and $j$ have frequencies such that $\omega_{i}-\omega_{j}<O(\epsilon)$. With $\omega_{i}=(1+\Omega) q_{i}-k_{i}$ for all $i$, the frequencies $q_{i}$ and $q_{j}$ have to obey

$$
q_{i}-q_{j}=\frac{k_{i}-k_{j}}{1+\Omega}+\frac{1}{1+\Omega} O(\epsilon) .
$$

That means for a mode with frequency $q_{j}$ a resonance to form an unstable triad is only possible if there is another mode with a frequency in an interval of size $O(\epsilon) /(1+\Omega)$ centered around $q_{j}+\left(k_{i}-k_{j}\right) /(1+\Omega)$. We consider first the case of small $\Omega$, then $q_{i}-q_{j} \approx k_{i}-k_{j}+O(\epsilon)$. If the frequencies are statistically uniformly distributed over the interval, and the matrix elements in Eq. (2.35) do not systematically vary with frequency then one will find on average one resonance with positive growth rate among a number $N$ of modes proportional to $1 / \epsilon$. Only a finite number of modes exist with an $n$ smaller than some limit $L$ : For every $n$ and $k$, there are $n-|k|$ modes if $k \neq 0$ and $n-1$ modes if $k=0$. For every $n$, the index $k$ has to obey $|k| \leq n$ so that there are $n^{2}-1$ modes of spatial degree $n$ and $N(L)$ modes exist with $n \leq L$, with $N(L)$ given by:

$$
N(L)=\sum_{n=1}^{L}\left(n^{2}-1\right)=\frac{1}{6} L(L+1)(2 L+1)-L .
$$

For large $L$, one has $N \propto L^{3}$, and in order for these modes to contain a resonance, one needs $L \propto \epsilon^{-1 / 3}$, independently of $\Omega$ or $\beta$. Figure 2.7 verifies this scaling. This figure 
has been obtained as follows: At fixed $\epsilon, 20$ equidistant points have been chosen in the interval of $\Omega$ ranging from -0.45 to 0.5 . The triad with the mode with the smallest $\mathrm{n}$ for which the growth rate is at least $0.1 \sigma_{\mathrm{ud}}$ and $0.8 \sigma_{\mathrm{ud}}$ was determined for every point. This minimal $n$, averaged over all points, is $\langle n\rangle$ and is shown in Fig. 2.7 as a function of $\epsilon$. The average $\langle n\rangle$ is a measure of the minimum $n$ that is typically necessary to obtain a resonance with a given $\epsilon$. This averaging procedure is necessary, because as it can be seen exemplarily for the viscous case in Fig. 2.11 the $n$ value of the modes which form a triad is scattered about a certain range of values. Because of the viscosity this range is limited. In Fig. 2.6 the $n$ values for the inviscid case are shown.

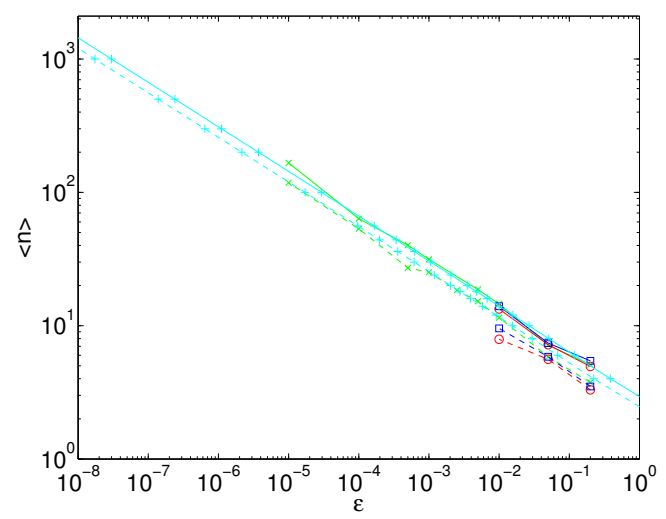

Figure 2.7.: Mean value of the spatial degree $\langle n\rangle$, for the modes with a growth rate of at least $0.1 \sigma_{u d}$ (dashed lines) or $0.8 \sigma_{u d}$ (solid lines) as a function of $\epsilon$ for $\beta=0$ (green $\mathrm{x}$ ), $\beta=2.8$ (red circles), and $\beta=18$ (blue squares), together with fits according to Eq.2.52. Credit: Clausen \& Tilgner, A\&A, 562, A25, 2014, reproduced with permission OESO.

Now we will consider the case of large $\Omega$. Positive growth rates are only possible for $q_{i} q_{j}<0$, as can be seen in Eq. (2.35). Additionally for $\Omega$ tending to infinity $q_{i}-q_{j}$ tends to zero, this two conditions imply that $q_{i}$ and $q_{j}$ both have to go to zero as $1 /|\Omega|$ for large $|\Omega|$. A large enough $n$ is necessary to find an absolute value below a certain border, as can be seen in Fig. 2.2. Therefore a minimal spatial degree $n_{\min }$ is necessary to find a resonance at a given $\Omega$. Furthermore the second mode frequency needs to lie in an interval of size $O(\epsilon) /(1+\Omega)$ for a positive growth rate. This additional condition is possibly met only for $n$ larger than $n_{\min }$.

For the determination of $n_{\min }$ we need a relationship between the smallest frequency and the spatial degree of the mode. As already stated the frequencies of inertial modes in a uniform density sphere can be calculated according to Eq. (29) from Wu (2005a), see Eq. (2.49). 


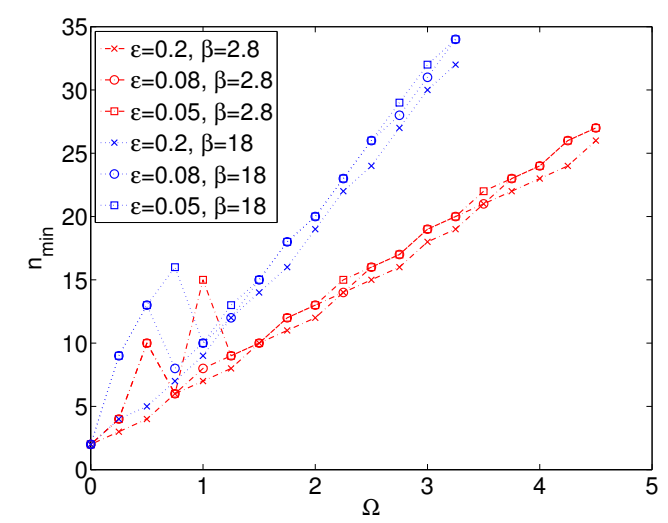

(a)

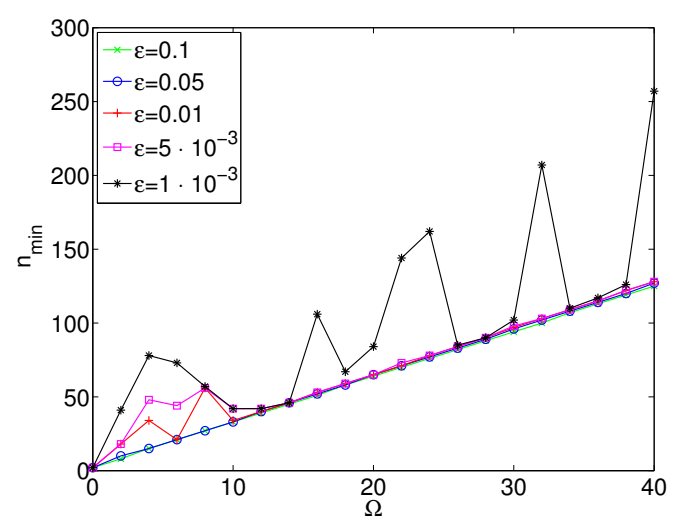

(b)

Figure 2.8.: Minimum value of $n, n_{\min }$, as a function of $\Omega$ for various $\epsilon$ and positive $\Omega$. $\beta=2.8$ and $\beta=18$ (a) and $\beta=0$ (b). Credit: Clausen \& Tilgner, A\&A, 562, A25, 2014, reproduced with permission $\odot$ ESO.

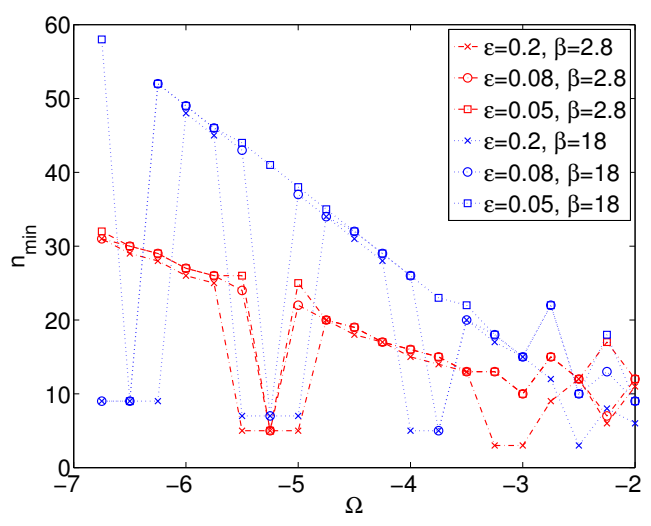

(a)

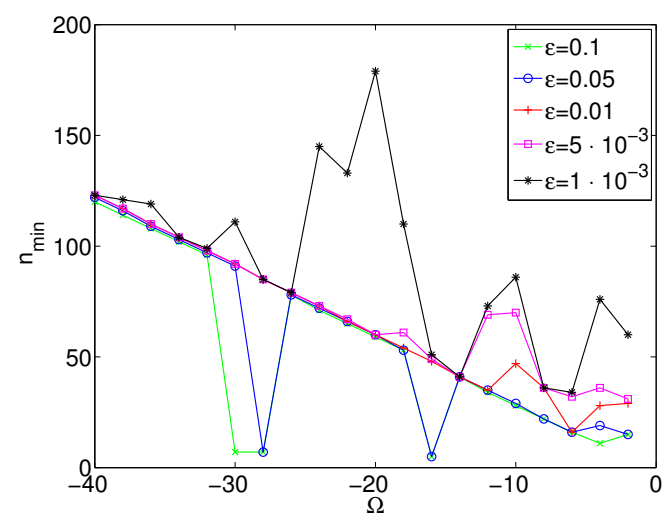

(b)

Figure 2.9.: Same as in Fig. 2.8 but for negative $\Omega$. Credit: Clausen \& Tilgner, A\&A, 562, A25, 2014, reproduced with permission CESO. 
For large $n$ the asymptotic expansion (Abramowitz and Stegun, 1972)

$$
\begin{gathered}
P_{n}^{k}(\cos \theta)=\frac{\Gamma(n+k+1)}{\Gamma(n+3 / 2)}\left(\frac{1}{2} \pi \sin (\theta)\right)^{-1 / 2} \\
\quad \times \cos \left[\left(n+\frac{1}{2}\right) \theta-\frac{\pi}{4}+\frac{k \pi}{2}\right]+O\left(n^{-1}\right)
\end{gathered}
$$

holds. We set $\zeta=\cos (\theta)$ and $\theta=\pi / 2+\epsilon_{\mathrm{w}}$ with $\epsilon_{\mathrm{w}}$ small for $\zeta$ small. A short calculation shows that for small $\zeta$ and large $n$, Eq. (2.49) is equivalent to

$$
\begin{array}{r}
-k \cos \left[\left(n+\frac{1}{2}\right) \epsilon_{\mathrm{w}}+\frac{(n+k) \pi}{2}\right] \\
=\left(n+\frac{1}{2}\right) \sin \left[\left(n+\frac{1}{2}\right) \epsilon_{\mathrm{w}}+\frac{(n+k) \pi}{2}\right] .
\end{array}
$$

For $n+k$ even this gives

$$
\begin{aligned}
& \epsilon_{\mathrm{w}} \approx \frac{-k}{\left(n+\frac{1}{2}\right)^{2}} \quad \text { for } \quad\left(n+\frac{1}{2}\right) \epsilon_{\mathrm{w}} \rightarrow 0, \\
& \epsilon_{\mathrm{w}} \approx \pm \frac{h_{\mathrm{a}} \pi}{n+\frac{1}{2}} \quad \text { for } \quad\left(n+\frac{1}{2}\right) \epsilon_{\mathrm{w}} \rightarrow \pm h_{\mathrm{a}} \pi,
\end{aligned}
$$

and for $n+k$ odd

$$
\epsilon_{\mathrm{w}} \approx \pm \frac{h_{\mathrm{a}} \pi}{2\left(n+\frac{1}{2}\right)} \quad \text { for } \quad\left(n+\frac{1}{2}\right) \epsilon_{\mathrm{w}} \rightarrow \pm \frac{h_{\mathrm{a}} \pi}{2},
$$

where $h_{\mathrm{a}}$ is an integer. For large $n$ and small $k$ the $\zeta$ with the smallest absolute value tends towards zero as $k /(n+1 / 2)^{2}$. We can classify these modes as "slow modes", because if the frequency of modes on any other branch of the dispersion relation in Fig. 2.2 tends to zero, it does so only in $1 /(n+1 / 2)$. The growing resonance is either between two slow modes, or at least one of the two inertial modes involved is not a slow mode. In the latter case, $n_{\min } \propto|\Omega|$, whereas in the former case, $n_{\min } \propto \sqrt{|\Omega|}$. However, since $\left|k_{1}-k_{2}\right|=2$, and choosing the indices such that $k_{2}>k_{1}$, a resonance between slow modes can only occur if $k_{1}=-1$ and $k_{2}=1$, and from Eq. (2.51) it can be deduce that $\Omega$ must be negative. In summary, the expectation is $n_{\min } \propto \Omega$ for positive $\Omega$, and for negative $\Omega$, too, except when modes with frequencies of approximately $k /(n+1 / 2)^{2}$ can resonate with each other. This scaling for positive $\Omega$ can be found in Fig. 2.8. Additionally from this figure a prefactor $d_{1}$ can be obtained with $n_{\text {min }}=d_{1} \Omega$, this prefactor depends on $\beta$. From the figure it can be deduced $d_{1}=3,5$ and 10 for $\beta=0,2.8$ and 18 , respectively. The reason why some points lie above the line $n_{\min }=d_{1} \Omega$ is the additionally condition that they must find a mode to resonate with in the interval $O(\epsilon) /(1+\Omega)$. For negative $\Omega$ (Fig. 2.9) points below $n_{\min }=d_{1} \Omega$ can be found which correspond to resonances between two slow 
modes. These always involve the azimuthal wavenumbers $k_{1}=-1$ and $k_{2}=1$. In most applications $\Omega>0$, therefore $n_{\min }$ is used in the estimation of dissipation effects below.

In the remaining part of this section we will examine viscous effects. Dissipation is taken into account according to Eqs. (2.37) and (2.38). At the $E k$ and $n$ under consideration, the first term corresponding to friction at the boundaries dominates in the equation for no slip boundary conditions, Eq. (2.38). Therefore the maximum growth rate in Fig. 2.10(b) is roughly given by $\sigma_{\mathrm{ud}}-h_{2}|1+\Omega| \sqrt{E k}$. Instability is expected if $\sigma_{\mathrm{ud}}-h_{2}|1+\Omega| \sqrt{E k}>0$, independently of $\beta$. Values of $h_{1}$ depend on the inertial mode and can vary by an order of magnitude, but much bigger is in practice the uncertainty in $E k$, therefore it is not necessary to determine the prefactor $h_{1}$ more accurately.

For free slip boundary conditions according to Eq. (2.37) only bulk dissipation matters. In this case dissipation always depends significantly on the spatial degree $n$. For the free slip case bigger $E k$ are possible in general because there is no term $\propto \sqrt{E k}$. Only for very large $\Omega$ or very small $\epsilon$, depending on $E k$, a high $n$ is necessary such that then the deviation between the growth rates for free slip and no slip boundary conditions becomes negligible.

For free slip boundary conditions the stability limit depends on the structure of the unstable mode and hence on $\beta$ and $\Omega$. As an estimate for the growth rate in this case we take the inviscid growth rate reduced by the decay rate of modes with a typical $n$ value. We take $\langle n\rangle$ as a typical value for small $|\Omega|$ and $n_{\text {min }}$ for large $|\Omega|$. A formula which interpolates between these two limits is $n=d_{1}|\Omega|+d_{2} \epsilon^{-1 / 3}$. For the parameters of Fig. 2.10 , only the term $d_{2} \epsilon^{-1 / 3}$ is important and one arrives at

$$
\sigma_{\mathrm{fs}}=\sigma_{\mathrm{ud}}-d_{2}^{2} \epsilon^{-2 / 3} E k,
$$

which is again independent of $\beta$. From Fig. 2.10(a), we deduce that $d_{2}$ is roughly 3.35 .

\subsubsection{Examples: Io's tides on Jupiter, the binary system V636 Centauri and the Earth}

How the results of the previous section can be used to determine if in a given planet or star the elliptical instability appears, is content of this section. It will be shown with help of three examples. The $0.85 M_{\odot}$ secondary is considered as the perturbing object within the binary system V636 Centauri, the Moon as the perturbing object for the Earth, and the tides on Jupiter raised by Io. The first problem we encounter is which boundary conditions are suitable. For the Earth's core it is clear that no-slip boundary conditions apply. In the two remaining examples we use free-slip conditions. According to a study by Tassoul 


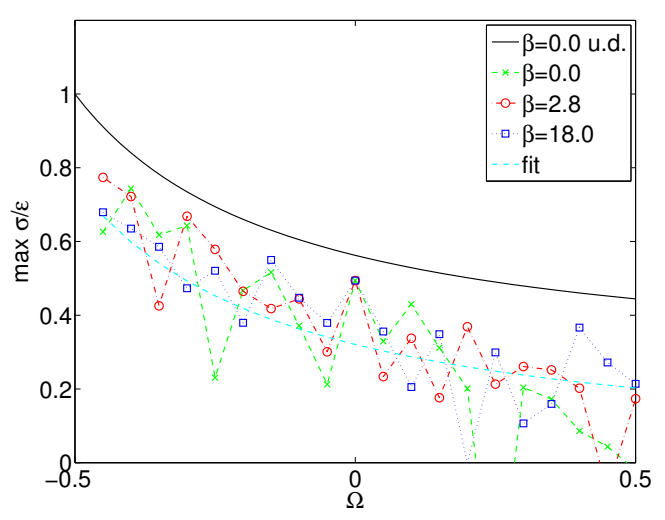

(a) $\epsilon=1 \times 10^{-2}, E k=1 \times 10^{-5}$

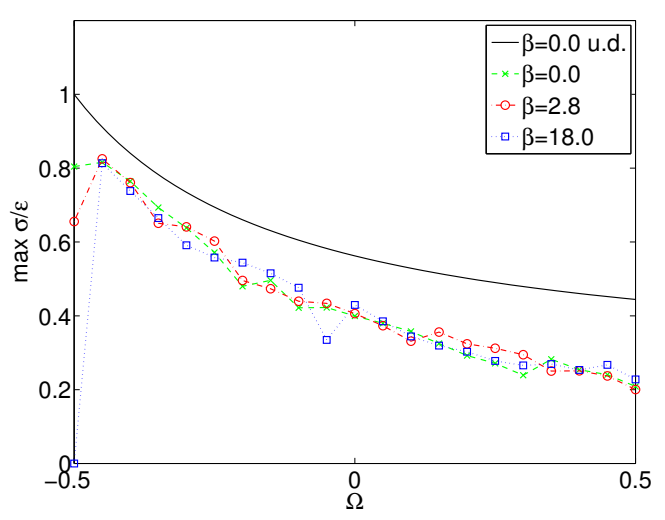

(b) $\epsilon=1 \times 10^{-2}, E k=1 \times 10^{-6}$

Figure 2.10.: Maximum growth rates as a function of $\Omega$ for $E k \neq 0$. For this figure we take into account all modes with a maximum $|k| \leq 10$ and $n \leq 40$. In (a) calculated for free slip boundary conditions according to Eq. (2.37). In (b) calculated for no slip boundary conditions according to Eq. (2.38). The black line in this figure is the growth rate given by Eq. (2.50). The cyan colored line in (a) is the growth rate given by Eq. (2.57) with $d_{2}=3.35$. Credit: Clausen \& Tilgner, A\&A, 562, A25, 2014, reproduced with permission CESO.

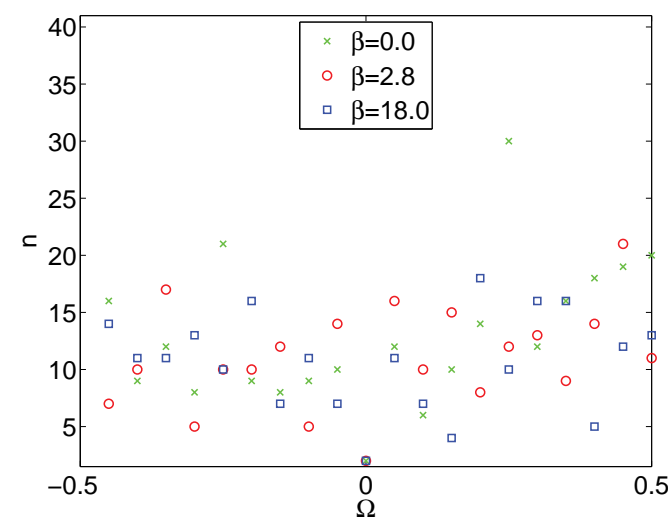

(a) $\epsilon=1 \times 10^{-2}, E k=1 \times 10^{-5}$

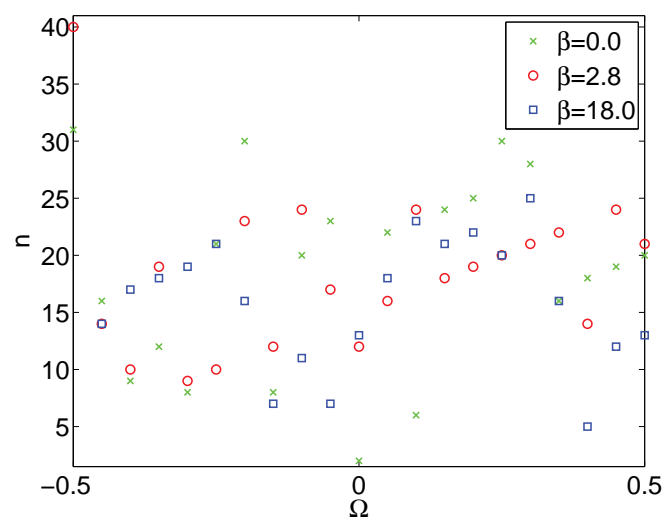

(b) $\epsilon=1 \times 10^{-2}, E k=1 \times 10^{-6}$

Figure 2.11.: The corresponding values of the spatial degree $n=n_{i}=n_{j}$ for the growth rates in Fig. 2.10. 
and Tassoul (1997) (see also Tassoul 1987, 1995) also with free slip boundary conditions a dissipation due to Ekman pumping proportional to $E k^{1 / 2}$ can appear, while Rieutord (1992) (see also Rieutord and Zahn 1997; Rieutord 2008) argues that the dissipation due to Ekman pumping scales always as $E k$ for free slip boundary conditions. If Tassouls stronger dissipation scaling applies also for the free slip case a damping rate according to Eq. (2.38) would be appropriate. But Tassouls derivation only applies if a linearization is tractable, this is not the case for our two examples (according to the condition in Eq. (19) of Tassoul and Tassoul 1997). The case Jupiter Io was treated in Tassoul and Tassoul (1997). There it is argued why their scaling not applies for Jupiter Io and in a analogous way it can be derived why it not applies for V636 Centauri. Although V636 Centauri is a binary star system with about equal components and therefore is a candidate for a stronger dissipation scaling according to Tassouls mechanism.

More problematic is how to deal with additional fluid motion, such as turbulent convection. According to at least one well established turbulence model, the Langranian-averaged Navier Stokes $\alpha$ (LANS- $\alpha$ ) turbulence model, the growth rate of the elliptical instability is enhanced for wavelengths of the inertial wave that are longer than the turbulence correlation length and suppressed for shorter wavelengths (Fabijonas and Holm, 2003). Whereby it can be suppressed such that a without turbulent motion instable flow gets stabilized. They considered the inviscid case, also the turbulent viscosity was set to zero. The maximal value of the growth rate with turbulent motion is $\sigma_{\mathrm{inv}}=\epsilon$.

Furthermore, in stellar and planetary objects in the region where turbulent convection takes place one has to consider a turbulent viscosity, due to the turbulent motion. This is generally much higher than the molecular viscosity. If the tidal period is shorter than the turn over time of turbulent eddies, the turbulent viscosity is attenuated. Several authors (Wu (2005b) and in a similar form Ogilvie and Lin (2007)) have used the following expression for the turbulent viscosity $\nu_{\mathrm{t} ; \mathrm{NR}}$ :

$$
\nu_{\mathrm{t}, \mathrm{NR}} \sim v_{\mathrm{cv}} l_{\mathrm{cv}} \frac{1}{1+\left(\omega_{\text {tide }} \tau_{\mathrm{cv}} /(2 \pi)\right)^{s_{\mathrm{e}}}}
$$

where $v_{\mathrm{cv}}, l_{\mathrm{cv}}$ and $\tau_{\mathrm{cv}}=l_{\mathrm{cv}} / v_{\mathrm{cv}}$ are the characteristic convection velocity, mixing length and turnover time and $\omega_{\text {tide }}=2\left|\Omega_{\mathrm{F}}\right|$ is the frequency of the tidal forcing. $s_{\mathrm{e}}$ is a constant, generally $s_{\mathrm{e}}=1$ or $s_{\mathrm{e}}=2$ is used. NR stands for non-rotating convection, because the effects of rotation on the convection is not incorporated into the formula above. How we will incorporate these effects is shown below.

We will use in this work $s_{\mathrm{e}}=1$ for $T_{\mathrm{f}} \gtrsim \tau_{\mathrm{cv}}$, with $T_{\mathrm{f}}=2 \pi / \omega_{\text {tide }}$, and $s_{\mathrm{e}}=2$ for $T_{\mathrm{f}} \ll \tau_{\mathrm{cv}}$, as suggested by Penev et al. (2009). The reasons for this will now be explained, according 
to the work of Penev et al. (2009) and Goldreich and Nicholson (1977). We consider first the case $T_{\mathrm{f}} \gtrsim \tau_{\mathrm{cv}}$, Komlogorov scaling is then not expected to apply, because turnover times on the order of $T_{\mathrm{f}}$ correspond then to eddies with sizes comparable or larger than the pressure scale height or density scale height. If $T_{\mathrm{f}} \gtrsim \tau_{\mathrm{cv}}$ a limit to $\nu_{t}$ is obtained by setting $s_{\mathrm{e}}=1$, this scaling was found in this regime by Penev et al. (2009) through numerical calculations. For $T_{\mathrm{f}} \ll \tau_{\mathrm{cv}}$ Kolmogorov scaling applies, a limit to $\nu_{t}$ is obtained by replacing $l_{\mathrm{cv}}$ by $v_{\mathrm{cv}} T_{\mathrm{f}}$, the distance over which the turbulent fluid can move in one tidal cycle, this would correspond to $s_{\mathrm{e}}=1$, ignoring the summand 1 in the denominator. But when $T_{\mathrm{f}} \ll \tau_{\mathrm{cv}}$ this is probably a gross overestimate of $\nu_{t}$, because turbulent eddies have lifetimes which are comparable to their turnover times and lifetimes $\tau_{\lambda}$ decrease with decreasing eddy size $\lambda$. Therefore the contribution to $\nu_{t}$ by the largest eddies is likely to be smaller than $v_{\mathrm{cv}} l_{\mathrm{cv}}\left(T_{\mathrm{f}} / \tau_{\mathrm{cv}}\right)$ by at least one additional factor of $\left(T_{\mathrm{f}} / \tau_{\mathrm{cv}}\right)$. Eddies with $\tau_{\lambda} \leq T_{\mathrm{f}}$ contribute an amount $\nu_{\lambda} \approx \lambda v_{\lambda}$ to the effective turbulent viscosity. Now assuming Kolmogorov scaling applies the turnover time of an eddy of size $\lambda$ is given by

$$
\tau_{\lambda} \approx \tau_{\mathrm{cv}}\left(\frac{\lambda}{l_{\mathrm{cv}}}\right)^{\frac{2}{3}}
$$

The typical velocity difference across such an eddy is

$$
v_{\lambda} \approx v_{\mathrm{cv}}\left(\frac{\lambda}{l_{\mathrm{cv}}}\right)^{\frac{1}{3}}
$$

Therefore it is

$$
\nu_{\lambda}=\lambda v_{\lambda}=l_{\mathrm{cv}} v_{\mathrm{cv}}\left(\frac{\tau_{\lambda}}{\tau_{\mathrm{cv}}}\right)^{2} .
$$

Among those eddies with $\tau_{\lambda} \leq T_{\mathrm{f}}$ those with $\tau_{\lambda} \approx T_{\mathrm{f}}$ have the most important contribution. These give rise to a turbulent viscosity

$$
\nu_{t} \approx l_{\mathrm{cv}} v_{\mathrm{cv}}\left(\frac{T_{\mathrm{f}}}{\tau_{\mathrm{cv}}}\right)^{2}
$$

That $s_{\mathrm{e}}=2$ should be used at small tidal periods is in accordance with results from Ogilvie and Lesur (2012).

The mixing length is estimated by $l_{\mathrm{cv}} \approx H$, with $H=-\frac{\mathrm{d} r}{d \ln \rho}=\frac{r^{-1}-r}{2 \beta}$ the density scale height. Therefore an order of magnitude estimation gives $l_{\mathrm{cv}} \sim \frac{R_{\mathrm{ce}}}{2 \beta}$. The convective velocity is approximated by $v_{\mathrm{cv}} \approx(\mathrm{F} / \rho)^{1 / 3}$, with the energy flux $\mathrm{F}=L /\left(4 \pi R_{\mathrm{ce}}^{2}\right)$ and $R_{\mathrm{ce}}$ the radius of the central body. An order of magnitude for $v_{\mathrm{cv}}$ is therefore obtained by using $v_{\mathrm{cv}} \approx\left(4 \pi R_{\mathrm{ce}}^{3} \mathrm{~F} /\left(3 m_{\mathrm{ce}}\right)\right)^{1 / 3}$, with $m_{\mathrm{ce}}$ the mass of the central body.

The effect of rotation on convection was until recently ignored in the calculation of the 
turbulent convection, but based on the work of Barker et al. (2014) in Mathis et al. (2014) it is described how to incorporate these effects. Barker et al. (2014) used high resolution non linear 3-D cartesian simulations of turbulent convection to confirm asymptotic scaling laws which were proposed by Stevenson (1979) for the regimes of slow and rapid rotation. The actual regime can be determined by the convective Rossby number defined as (Stevenson, 1979)

$$
R o_{c}=\left(\frac{v_{\mathrm{cv}}}{l_{\mathrm{cv}} 2 \Omega_{\mathrm{spin}}}\right)=\frac{\tau_{\mathrm{spin}}}{\tau_{\mathrm{cv}}},
$$

with $\tau_{\text {spin }}=1 /\left(2 \Omega_{\text {spin }}\right) . \quad R o_{c} \ll 1$ and $R o_{c} \gg 1$ correspond to rapid and slow rotation regimes, respectively. For Jupiter and the primary of V636 Centauri it is $R o_{c} \ll 1$ (see Table 2.1), so these objects lie in the regime of rapidly rotating convection. In this regime it is $\nu_{\mathrm{t} ; \mathrm{RC}} / \nu_{\mathrm{t} ; \mathrm{NR}} \approx 3\left(R o_{c}\right)^{4 / 5}$ (Mathis et al., 2014), with $\nu_{\mathrm{t} ; \mathrm{RC}}$ the turbulent viscosity for which the effects of rapid rotation on convection are taken into account.

Table 2.1 lists for the three examples the growth rate for an inviscid fluid, and the damping rates computed for molecular and turbulent viscosities. In the no slip case the damping rate $\chi$ is given by $\chi=(1+\Omega) \sqrt{E k}$ according to Eq. (2.38). We neglect the volume damping term in both cases because $\Omega$ and $E k$ are small enough. In the free slip case, $\chi=d_{2}^{2} \epsilon^{-2 / 3} E k$ according to Eq. (2.37). For $d_{2}$ we choose 3.35 , the value extracted from Fig. 2.10(a). We neglect the terms which are relevant if $\Omega$ is large because $\Omega$ is small enough in both cases. The ellipticity is calculated according to

$$
\epsilon=\frac{1}{2} \frac{m_{\mathrm{p}}}{m_{\mathrm{ce}}}\left(\frac{R_{\mathrm{ce}}}{a_{\mathrm{sm}}}\right)^{3}
$$

with $m_{\mathrm{p}}$ the mass of the perturbing body and $a_{\mathrm{sm}}$ the distance between the central and the perturbing body. The Ekman number is calculated according to

$$
E k=\frac{\nu}{\Omega_{\text {spin }} R_{E}^{2}},
$$

with $\Omega_{\text {spin }}=\Omega_{\mathrm{F}}+\Omega_{\mathrm{p}}$ and $R_{E}=R_{c o}$ the radius at the edge of the outer conducting region in the case of the Earth and the Jupiter-Io system and $R_{E}=R_{\mathrm{ce}}$ in the case V636 Centauri.

Instability is expected if the inviscid growth rate exceeds the viscous damping in Table 2.1. Table 2.2 summarizes the results of different modeling assumptions. If one only takes into account molecular viscosity the flow is unstable in Jupiter and the primary of V636 Centauri for all cases. In practice, the turbulence model decides on whether instability is predicted or not. Unfortunately, we have no turbulence model we can safely rely upon. 


\begin{tabular}{llll}
\hline \hline Constant & Earth-Moon & Jupiter-Io & V636 Cen. \\
\hline$R_{\text {ce }}(\mathrm{m})$ & $6.37 \times 10^{6} \mathrm{a}$ & $6.99 \times 10^{7} \mathrm{a}$ & $7.08 \times 10^{8} \mathrm{~b}$ \\
$R_{\mathrm{co}}(\mathrm{m})$ & $3.47 \times 10^{6 \mathrm{c}}$ & $5.71 \times 10^{7 \mathrm{c}}$ & $\ldots$ \\
$m_{\mathrm{ce}}(\mathrm{kg})$ & $5.97 \times 10^{24 \mathrm{c}}$ & $1.90 \times 10^{27 \mathrm{c}}$ & $2.09 \times 10^{30 ~ \mathrm{~b}}$ \\
$a_{\mathrm{sm}}(\mathrm{m})$ & $3.84 \times 10^{8 \mathrm{c}}$ & $4.22 \times 10^{8 \mathrm{c}}$ & $9.57 \times 10^{9} \mathrm{~d}$ \\
$m_{\mathrm{p}}(\mathrm{kg})$ & $7.35 \times 10^{22 \mathrm{c}}$ & $8.91 \times 10^{22 \mathrm{c}}$ & $1.70 \times 10^{30 \mathrm{~b}}$ \\
$L(\mathrm{~W})$ & $\ldots$ & $3.34 \times 10^{17 \mathrm{e}}$ & $4.31 \times 10^{26} \mathrm{~b}$ \\
$\nu_{\mathrm{m}}\left(\mathrm{m}^{2} \mathrm{~s}^{-1}\right)$ & $1.4 \times 10^{-6 \mathrm{f}}$ & $3 \times 10^{-7} \mathrm{e}$ & $10^{-4} \mathrm{~g}$ \\
$\Omega_{\mathrm{spin}}\left(\mathrm{day}^{-1}\right)$ & $2 \pi$ & $2 \pi / 0.41^{\mathrm{h}}$ & $2 \pi / 3.96^{\mathrm{i}}$ \\
$\Omega_{\mathrm{p}}\left(\right.$ day $\left.^{-1}\right)$ & $2 \pi / 27.32$ & $2 \pi / 1.77^{\mathrm{h}}$ & $2 \pi / 4.28^{\mathrm{b}}$ \\
$\Omega$ & 0.0380 & 0.305 & 12.40 \\
$\epsilon$ & $2.8 \times 10^{-8}$ & $1.1 \times 10^{-7}$ & $1.6 \times 10^{-4}$ \\
$E k_{\mathrm{m}}$ & $1.6 \times 10^{-15}$ & $5.2 \times 10^{-19}$ & $1.1 \times 10^{-17}$ \\
$\beta$ & $\ldots$ & 1.5 & 18 \\
$s_{\mathrm{e}}$ & $\ldots$ & 2 & 1 \\
$E k_{\text {turb;NR }}$ & $\ldots$ & $1.6 \times 10^{-13}$ & $6.3 \times 10^{-5}$ \\
$R o_{c}$ & $\ldots$ & $1.94 \times 10^{-5}$ & $5.05 \times 10^{-2}$ \\
$E k_{\text {turb} ; \mathrm{RC}}$ & $\ldots$ & $8.44 \times 10^{-17}$ & $1.74 \times 10^{-5}$ \\
$\sigma_{\text {ud }}$ & $1.6 \times 10^{-8}$ & $5.1 \times 10^{-8}$ & $4.4 \times 10^{-5}$ \\
$\chi_{\mathrm{m}}$ n.s. & $4.2 \times 10^{-8}$ & $\ldots$ & $\ldots$ \\
$\chi_{\text {turb;NR }}$ f.s. & $\ldots$ & $8.3 \times 10^{-8}$ & 0.24 \\
$\chi_{\text {turb;RC }}$ f.s. & $\ldots$ & $4.22 \times 10^{-11}$ & $6.5 \times 10^{-2}$ \\
\hline
\end{tabular}

Table 2.1.: Parameters for the example objects. $\nu_{\mathrm{m}}$ is the molecular viscosity, $E k_{\mathrm{m}}$ the Ekman number based only on the molecular viscosity, and $E k_{\text {turb;NR }}$ and $E k_{\text {turb;RC }}$ the Ekman number based on the turbulent viscosity without and with the effects of rotation on convection, respectively, (the molecular viscosity is negligible in comparison to the turbulent in these examples). $\chi_{\mathrm{m}}, \chi_{\mathrm{turb} ; \mathrm{NR}}$, and $\chi_{\mathrm{turb} ; \mathrm{RC}}$ are damping constants calculated with $E k_{\mathrm{m}}, E k_{\mathrm{turb} ; \mathrm{NR}}$, and $E k_{\mathrm{turb} ; \mathrm{RC}}$, respectively. n.s. and f.s. stands for no slip and free slip boundary conditions, respectively.

References. $\quad{ }^{\text {a }}$ Archinal et al. (2011); $\quad{ }^{\mathrm{b}}$ Clausen et al. (2009); $\quad{ }^{\mathrm{c}}$ Wicht and Tilgner (2010); d Calculated according to Kepler's third law, with values given in Clausen et al. (2009); $\quad$ e Guillot et al. (2004); $\quad{ }^{\mathrm{f}}$ DeWijs et al. (1998); $\quad{ }^{\mathrm{g}}$ Miesch (2005);

${ }^{\mathrm{h}} \mathrm{Wu}(2005 \mathrm{~b}) ; \quad{ }^{\mathrm{i}}$ Calculated according to $v=\Omega_{\text {spin }} \cdot R_{\text {ce }}$ with $v$ the equatorial velocity given in Clausen et al. (2009)

\begin{tabular}{cccc}
\hline \hline J-I: $\nu_{\mathrm{t} ; \mathrm{NR}}$ & J-I: $\nu_{\mathrm{t} ; \mathrm{RC}}$ & VC: $\nu_{\mathrm{t} ; \mathrm{NR}}$ & VC: $\nu_{\mathrm{t} ; \mathrm{RC}}$ \\
\hline uncertain & unstable & stable & stable \\
\hline
\end{tabular}

Table 2.2.: Summary of the stability characteristics of the flow for the examples Jupiter-Io (JI) and V636 Centauri (VC). The top row indicates if turbulent viscosity without the effects of rotation on the convection has been used $\left(\nu_{\mathrm{t} ; \mathrm{NR}}\right)$ or with these effects $\left(\nu_{\mathrm{t} ; \mathrm{RC}}\right)$ in the computation of the viscous damping. We used free slip boundary conditions. 


\subsection{Conclusion and discussion}

We computed the linear stability limit of tidal flow within the anelastic approximation through a perturbation calculation in which the small parameter is the deformation of the central body from spherical shape. The instabilities are described as superpositions of two inertial modes of the rotating sphere. The perturbation calculation is tractable if the initially three dimensional problem of the computation of the inertial modes is separable and reduces to the solution of an ordinary differential equation. This is the case if the density profile obeys a power law. For an inviscid fluid, the growth rate of the combination of two chosen modes depends on the density profile, but the growth rate maximized over all possible combinations does not. Furthermore, the maximum growth rate is the same as the one known for elliptical instability in infinitely extended, incompressible fluids. The fact that one finds the same maximum growth rates in so widely different situations justifies some tolerance towards modeling assumptions. For example, we restricted our analysis to density profiles obeying power laws and to the boundary condition that the velocity normal to the boundary be zero at the surface. After the calculations presented here, we expect that different modes will be found to be the most unstable for more realistic assumptions, but that the stability limit of the flow will stay the same. In the same fashion, we do not expect the particular choice of the major axis parallel to the rotation axis in Eq. (2.19) to affect the stability limit.

We added viscous effects empirically. If friction at a solid boundary dominates dissipation, the stability is easily determined as a function of the rotation rates of the central body and the tidal companion, the tidal deformation, and the Ekman number. If on the other hand bulk dissipation dominates, the dissipation depends on the flow structure of the unstable modes and therefore on all details of the model, in particular the density profile. However, dissipation depends mostly on the spatial degree which on average obeys a simple scaling law as a function of tidal deformation, so that an estimate of the stability limit is still possible.

Viscous damping also depends on boundary conditions, and models of an object's interior help to decide on which approximation to the viscous damping is most accurate. For instance, the dissipation in Ekman layers usually dominates if a solid core is present. However, if one wants to predict the stability limit of a particular astrophysical object, the biggest uncertainty comes from a possible turbulent viscosity. Progress in the calculation of tidal dissipation thus mostly hinges on advances in the treatment of turbulence. 
3 Dissipation of rocky planets for strong tidal forcing 
For the orbital evolution of objects in a planetary system it is important to know how much energy is dissipated due to tides. Usually tidal dissipation is parameterized by the so called tidal quality factor $Q$ or the modified tidal quality factor $Q^{\prime}$ (see Sect. 3.1.5). In exoplanetary systems for terrestrial planets one often calculates with a $Q^{\prime}$ value of 100 which approximates the modified tidal quality factor of the Earth (Murray and Dermott, 1999). The Earth has $Q=12$ (Murray and Dermott, 1999) which is on the same order as the quality factor of other terrestrial planets in our solar system. According to a study by Goldreich and Soter (1966) these lie in the range $10 \leq Q \leq 190$.

A study by Hansen and Murray (2015) suggests that a $Q^{\prime}$ of approximately 10 is a reasonable a priori estimate for terrestrial planets. They obtained with this value the best match with the observed eccentricity distribution of terrestrial exoplanets, assuming a scenario for planet formation (which fixes the initial conditions for the orbital evolution) as studied in Hansen and Murray (2013).

A recent paper by Henning and Hurford (2014) investigated how $Q^{\prime}$ depends on the orbital period and on the interior of the planet, they also considered multilayer planets with the possibility of oceans and ice regions.

That it is inappropriate to approximate super Earth tidal quality factors with that of the solid Earth was already claimed by Efroimsky (2012), because due to a higher self gravitation in a super Earth compared to an Earth mass object tidal damping in super Earth is less efficient. But for strong tidal forcing, if a stable equilibrium between tidal heat production and heat transport is possible also the scaling of heat transport and heat production with radius needs to be considered.

The $Q^{\prime}$ parameterization is controversial, it not only depends on the size of the object, it additionally depends on the frequency (Efroimsky and Lainey, 2007). The relative small tidal quality factor of the Earth comes from the fact that on the Earth the dissipation due to the ocean is high, a small quality factor means high tidal dissipation. If a liquid ocean out of water as on the Earth or lava may be present an estimate of tidal dissipation becomes more complicated.

In this study only planets with strong tidal forcing are investigated, because if the forcing is strong enough a stable equilibrium between heat transport due to convection or melt migration and tidal heat production is possible (see, e.g., Henning et al. 2009). The influence of convection as opposed to melt migration as possible heat transport mechanism is investigated. Jupiters moon Io and the exoplanet Corot $7 \mathrm{~b}$ are used as objects of study. One aim of this study is to investigate how the quality factor depends on the size of the 
terrestrial planet, the thickness of the asthenosphere, and on the orbital parameters and thereby investigate which $Q^{\prime}$ value is reasonable for a rocky planet.

\subsection{Theoretical background}

\subsubsection{Equilibrium tide}

The amplitude of the tidal deformation and the associated energy are determined by the tide raising potential and by the internal structure of the satellite. On a synchronous eccentric orbit, the time varying potential is given to first order in eccentricity by

$$
\begin{aligned}
\Phi_{t} & =r^{2} \Omega_{\mathrm{p}}^{2} e\left(-\frac{3}{2} P_{2}^{0}(\cos \theta) \cos \left(\Omega_{\mathrm{p}} t\right)\right) \\
& +r^{2} \Omega_{\mathrm{p}}^{2} e\left(\frac{1}{4} P_{2}^{2}(\cos \theta)\left[3 \cos \Omega_{\mathrm{p}} t \cos 2 \varphi+4 \sin \Omega_{\mathrm{p}} t \sin 2 \varphi\right]\right),
\end{aligned}
$$

where $r$ is the radius from the center of mass of the planet, $\Omega_{\mathrm{p}}$ the orbital angular velocity, $e$ the eccentricity of the orbit, $\theta$ and $\varphi$ are the colatitude and longitude with zero longitude at the near pole, $t$ is the time, $P_{2}^{0}$ and $P_{2}^{2}$ are associated Legendre polynomials. Throughout this chapter, "planet" denotes the tidally deformed body, be it a planet or a moon. Below we will see the problem simplifies if coordinates are chosen such that the problem is rotationally symmetric and the solution has then only to be expanded in Legendre polynomials. Therefore, we introduce $\vartheta$ the angle between the position vector and the line joining the center of the planet and the guiding center. The guiding center is the point which rotates about the occupied focus of the ellipse (where the tidal partner is placed) in a circle of radius $a_{\mathrm{sm}}$, the semi major axis, and with angular speed $\Omega_{\mathrm{p}}$ (Murray and Dermott, 1999). Additionally we introduce $\vartheta^{\prime}$ the angle between the position vector and the vector of the point $[\theta=\pi / 2, \varphi=\pi / 4]$. Now the potential can be written as (Murray and Dermott, 1999)

$$
\Phi_{t}=r^{2} \Omega_{\mathrm{p}}^{2} e\left[3 P_{2}(\cos (\vartheta)) \cos \left(\Omega_{\mathrm{p}} t\right)+4 P_{2}\left(\cos \vartheta^{\prime}\right) \sin \left(\Omega_{\mathrm{p}} t\right)+2 P_{2}(\cos \theta) \sin \left(\Omega_{\mathrm{p}} t\right)\right]
$$

with $P_{2}$ the Legendre polynomial of second degree.

In this study planets for which the tidal dissipation can be approximated by a calculation of the equilibrium tide are considered, as written in the introduction to this chapter. The dissipation due to the equilibrium tide for a tidally locked object on an elliptic orbit can 
be calculated according to (Segatz et al., 1988)

$$
D_{\mathrm{T}}=-\frac{21}{2} \Im\left(k_{2}\right) \frac{\left(\Omega_{\mathrm{p}} R_{\mathrm{p}}\right)^{5}}{G} e^{2},
$$

with $\Im\left(k_{2}\right)$ the imaginary part of the tidal Love number, $G$ the gravitational constant and $R_{\mathrm{p}}$ planet radius. For our purpose Eq. (3.3) is accurate enough, but for high eccentricities the equation underestimates tidal heating (Leconte et al., 2010). The factor $\Im\left(k_{2}\right)$ can be calculated similarly to the elastic spheroidal oscillations for a layered sphere (Alterman et al., 1959; Takeushi and Saito, 1972). If the planet is perfectly elastic no energy dissipation would take place, but planetary interiors for typical tidal forcing periods behave like viscoelastic bodies. The viscoelastic problem can be solved with the help of the correspondence principle (Biot, 1954), which allows one to extend the elastic problem formulation to the resolution of an equivalent viscoelastic problem.

The Maxwell model is used in this study for the viscoelastic interior. In this model $k_{2}=k_{2}\left(\eta, \mu, \Omega_{\mathrm{p}}, \rho, g, R_{\mathrm{p}}\right)$, with $\eta$ dynamic viscosity, $\mu$ shear modulus, $\rho$ density and $g$ gravitational acceleration. The Maxwell model should be valid as long as $\eta / \mu \lesssim 2 \cdot \pi / \Omega_{\mathrm{p}}$, for higher orbital angular velocities an effective viscosity can be introduced (Behounkova et al., 2013), this was not necessary in this study.

We follow the proceeding described in Sabadini and Vermeersen (2004) to derive the governing system of ordinary differential equations (ODEs). But opposed to them we solve these ODEs with a shooting method, they used a propagator matrix method. The here used notation follows Takeushi and Saito (1972). The equations require the density $\rho, \mu$ and $\eta$ to be given as a function of radius.

For spheroidal oscillations, one has to consider the momentum and the Poisson equation. Contrary to the formulation in Takeushi and Saito (1972), we consider the planetary material as incompressible and the inertial term will be neglected. The former is justified by calculations in Tobie et al. (2005) and own calculations (see Fig. 3.3), which show that the results depend only slightly on the bulk modulus. The latter is justified due to the fact that the excitation periods are on the order of days, but the natural period of the objects which will be investigated are $\sim 117 \mathrm{~min}$ and $\sim 82 \mathrm{~min}$ for the case of Io and Corot $7 \mathrm{~b}$, respectively. These periods are calculated for an incompressible homogeneous fluid sphere, calculated according to the classical formula of Lord Kelvin (see e.g. Dahlen and Tromp 1998)

$$
\omega_{\mathrm{k}}^{2}=\frac{8}{3} \frac{\pi l_{\mathrm{k}}\left(l_{\mathrm{k}}-1\right) G \rho}{2 l_{\mathrm{k}}+1},
$$

for a rough estimate, with degree $l_{\mathrm{k}}=2$ for the longest period. In a fluid sphere the only restoring force is the gravity, so especially in the less massive Jupiter moon Io the effects 
of elasticity would lead to a higher eigenfrequency than our estimates above.

We now follow the steps in Sabadini and Vermeersen (2004) (details can be found therein) to derive the governing system of ODEs. Conservation of linear momentum requires that the body forces $\mathbf{F}$ per unit mass acting on the element of the body are balanced by the stresses that act on the surface of the element. For the stress tensor $\boldsymbol{\sigma}$ acting on an infinitesimal block with density $\rho$ :

$$
\nabla \cdot \boldsymbol{\sigma}+\rho \mathbf{F}=0
$$

The stress tensor is the sum of the initial pressure plus a perturbation $\boldsymbol{\sigma}^{\prime}$ :

$$
\boldsymbol{\sigma}=\boldsymbol{\sigma}^{\prime}-p_{0} \mathbf{I}
$$

$\boldsymbol{\sigma}^{\prime}$ is a tensor and describes non-hydrostatic stress, related to the strain, $p_{0}$ is the hydrostatic pressure and $\mathbf{I}$ the identity matrix. At a fixed point in space the pressure change due to an elastic displacement $\boldsymbol{\xi}$ at time $t_{0}$ is given by

$$
p_{0}\left(t_{0}+\delta t\right)-p_{0}\left(t_{0}\right)=-\boldsymbol{\xi} \cdot \nabla p_{0}
$$

After the elastic displacement, the linear momentum Eq. (3.5) reads with $p_{0}\left(t_{0}+\delta t\right)$ instead of $p_{0}\left(t_{0}\right)$,

$$
\nabla \cdot \boldsymbol{\sigma}^{\prime}-\nabla p_{0}\left(t_{0}\right)-\nabla(\rho g \boldsymbol{\xi} \cdot \hat{\mathbf{r}})+\rho \mathbf{F}=0,
$$

where we used that the initial pressure is given by $\nabla p_{0}=-\rho g \hat{\mathbf{r}}$, where $\hat{\mathbf{r}}$ is the unit vector, positive outward from the planetary center. The force $\mathbf{F}$ can be split into all kinds of forcings and loads. For our case it is the sum of gravity and tidal force which can be written as a gradient field of a potential $\Phi_{\mathrm{f}}$, with $\mathbf{F}=-\nabla \Phi_{\mathrm{f}}$. $\Phi_{\mathrm{f}}$ can be written as $\Phi_{\mathrm{f}}=\Phi_{0}+\Phi^{\prime}, \Phi_{0}$ is the field in the initial state and $\Phi^{\prime}$ is the perturbation of the gravitational potential due to mass redistribution and the tidal potential. The linearized equation of momentum becomes

$$
\nabla \cdot \boldsymbol{\sigma}^{\prime}-\nabla(\rho g \boldsymbol{\xi} \cdot \hat{\mathbf{r}})-\rho \nabla \Phi^{\prime}=0
$$

since the second term of Eq. (3.8) is canceled by the term $-\rho \nabla \Phi_{0}$. The perturbation potential $\Phi^{\prime}$ satisfies the Poisson equation, which because of incompressibility reduces to the Laplace equation

$$
\nabla^{2} \Phi^{\prime}=0
$$

Further steps on these two governing equations can be found in Appendix D and E. After performing the steps in Appendix D the equation of motion and the Laplace equation can 
be written in terms of six functions $y_{i}(i=1,2, . ., 6)$ to calculate spheroidal oscillations

$$
\begin{aligned}
\frac{\mathrm{d} y_{1}}{\mathrm{~d} r} & =-\frac{1}{r}\left[2 y_{1}-l(l+1) y_{3}\right] \\
\frac{\mathrm{d} y_{2}}{\mathrm{~d} r} & =\frac{4}{r}\left(\frac{3 \mu}{r}-\rho g\right) y_{1}-\frac{l(l+1)}{r}\left(\frac{6 \mu}{r}-\rho g\right) y_{3}+\frac{l(l+1)}{r} y_{4}+\frac{\rho(l+1)}{r} y_{5}-\rho y_{6}, \\
\frac{\mathrm{d} y_{3}}{\mathrm{~d} r} & =\frac{1}{\mu} y_{4}+\frac{1}{r}\left(y_{3}-y_{1}\right), \\
\frac{\mathrm{d} y_{4}}{\mathrm{~d} r} & =-\frac{1}{r}\left(\frac{6 \mu}{r}-\rho g\right) y_{1}-\frac{1}{r} y_{2}+\frac{2\left(2 l^{2}+2 l-1\right) \mu}{r^{2}} y_{3}-\frac{3}{r} y_{4}-\frac{\rho}{r} y_{5}, \\
\frac{\mathrm{d} y_{5}}{\mathrm{~d} r} & =y_{6}+4 \pi G \rho y_{1}-\frac{l+1}{r} y_{5}, \\
\frac{\mathrm{d} y_{6}}{\mathrm{~d} r} & =\frac{l-1}{r}\left(y_{6}+4 \pi G \rho y_{1}\right)+\frac{4 \pi G \rho}{r}\left[2 y_{1}-l(l+1) y_{3}\right],
\end{aligned}
$$

with $l$ the degree of the spherical harmonic in the solution ansatz. The $y_{i}$ 's are coefficients in radius for an ansatz to solve the spheroidal oscillations problem in form of a spherical harmonic representation (see (D.7)). The ansatz for the tidal problem consists only of Legendre polynomials of second degree because the tidal potential can be written in form of only these polynomials, see Eq. (3.2).

The boundary conditions for the coefficients $y_{i}$ are

$$
y_{1}(0)=0, \quad y_{3}(0)=0, \quad y_{5}(0)=0,
$$

at the center, and

$$
y_{2}\left(R_{\mathrm{p}}\right)=0, \quad y_{4}\left(R_{\mathrm{p}}\right)=0, \quad y_{6}\left(R_{\mathrm{p}}\right)=\frac{2 n+1}{R_{\mathrm{p}}},
$$

at the surface.

$y_{1}$ and $y_{3}$ are associated with radial and tangential displacement, $y_{2}$ and $y_{4}$ with radial and tangential stresses, respectively. $y_{5}$ is associated with the gravitational potential and $y_{6}$ is defined such that the continuity of the gradient of the gravitational potential is taken into account.

It is $\mu=0$ in a liquid layer. The proceeding with this circumstance is analogue to the proceeding in Saito (1974). The equations of equilibrium in the radial and transversal 
directions and Poisson's equation can be written as

$$
\begin{aligned}
& \rho \frac{\mathrm{d} y_{5}}{\mathrm{~d} r}+\rho g X-\rho \frac{\mathrm{d}}{\mathrm{d} r}\left(g y_{1}\right)+\frac{\mathrm{d} y_{2}}{\mathrm{~d} r}=0, \\
& y_{2}=\rho\left(g y_{1}-y_{5}\right), \\
& \frac{\mathrm{d}^{2} y_{5}}{\mathrm{~d} r^{2}}+\frac{2}{r} \frac{\mathrm{d} y_{5}}{\mathrm{~d} r}-\frac{l(l+1)}{r^{2}} y_{5}=4 \pi G\left(\frac{\mathrm{d} \rho}{\mathrm{d} r} y_{1}+\rho X\right),
\end{aligned}
$$

with $X$ as the radial factor of dilation. These equations are the compressible counterpart of (Eqs. (D.10a), (D.10b) and (D.11)). This time we consider compressibility because we want to derive boundary conditions at the core mantle boundary, where the density has a jump discontinuity. The first equation of Eqs. (3.14) together with the differentiated second equation gives

$$
\rho g X=\frac{\mathrm{d} \rho}{\mathrm{d} r}\left(y_{5}-g y_{1}\right)=-\frac{\mathrm{d} \rho}{\mathrm{d} r} \frac{y_{2}}{\rho}
$$

and by making use of the third

$$
\frac{\mathrm{d}^{2} y_{5}}{\mathrm{~d} r^{2}}+\frac{2}{r} \frac{\mathrm{d} y_{5}}{\mathrm{~d} r}-\frac{l(l+1)}{r^{2}} y_{5}=\frac{\mathrm{d} \rho}{\mathrm{d} r} \frac{4 \pi G}{g} y_{5} .
$$

From the last equation it can be deduced that the potential $y_{5}$ is decoupled from displacement when $\mu=0$. For a given density distribution, we might be able to integrate Eq. (3.16), provided that $d y_{5} / d r$ is continuous in the liquid layer. But $d y_{5} / d r$ is not continuous when $\rho$ has a jump discontinuity. To circumvent this difficulty, a new variable will be introduced (details see Saito (1974))

$$
y_{7}=y_{6}+\frac{4 \pi G}{g} y_{2}=\frac{\mathrm{d} y_{5}}{\mathrm{~d} r}+\left(\frac{l+1}{r}-\frac{4 \pi G \rho}{g}\right) y_{5},
$$

this variable is continuously everywhere. Eq. (3.16) can now be rewritten in terms of $y_{5}$ and $y_{7}$ as two first order differential equations

$$
\begin{aligned}
\frac{\mathrm{d} y_{5}}{\mathrm{~d} r} & =\left(\frac{4 \pi G \rho}{g}-\frac{l+1}{r}\right) y_{5}+y_{7}, \\
\frac{\mathrm{d} y_{7}}{\mathrm{~d} r} & =\frac{2(l-1)}{r} \frac{4 \pi G \rho}{g} y_{5}+\left(\frac{l-1}{r}-\frac{4 \pi G \rho}{g}\right) y_{7} .
\end{aligned}
$$

This two equations will be integrated in the liquid core starting from initial values

$$
y_{5}(r)=r^{l} \quad r y_{7}(r)=2(l-1) r^{l}
$$

The integration can not be started at $r=0$ because then the differential equation will become singular at $r=0$. But for the case of this work, a numerical integration in 
the liquid core is not necessary because the liquid core is assumed to be homogeneous therefore the relations in Eq. (3.19) are solutions throughout the whole core. Except $y_{3}$ the $y_{i}$ 's should be continuous at $R_{\mathrm{c}}$, the core mantle boundary. $y_{4}$ is zero in the liquid because it is related to the tangential stress. $y_{1}, y_{2}$ and $y_{6}$ can not be determined by Eqs. (3.18), they are related to each other through the second equation in Eqs. (3.14) and Eq. (3.17), therefore only one is independent. The values $y_{1}\left(R_{\mathrm{c}}\right)$ and $y_{3}\left(R_{\mathrm{c}}\right)$ are taken as constants of integration. In the solid region there exist three independent solutions, under the condition of regularity at $r=0$. The boundary conditions at $R_{\mathrm{c}}$ are decomposed into three independent sets

$$
\begin{array}{ll}
\text { set } 1: & y_{11}^{\mathrm{so}}\left(R_{\mathrm{c}}\right)=0 \quad y_{21}^{\mathrm{so}}\left(R_{\mathrm{c}}\right)=-\rho^{\mathrm{li}}\left(R_{\mathrm{c}}\right) y_{51}^{\mathrm{li}}\left(R_{\mathrm{c}}\right) \\
& y_{51}^{\mathrm{so}}\left(R_{\mathrm{c}}\right)=y_{51}^{\mathrm{li}}\left(R_{\mathrm{c}}\right) \quad y_{61}^{\mathrm{so}}\left(R_{\mathrm{c}}\right)=y_{71}^{\mathrm{li}}\left(R_{\mathrm{c}}\right)+\frac{4 \pi G \rho^{\mathrm{li}}\left(R_{\mathrm{c}}\right)}{g\left(R_{\mathrm{c}}\right)} y_{51}^{\mathrm{li}}\left(R_{\mathrm{c}}\right) \\
\text { set } 2: & y_{12}^{\mathrm{so}}\left(R_{\mathrm{c}}\right)=1 \quad y_{22}^{\mathrm{so}}\left(R_{\mathrm{c}}\right)=\rho^{\mathrm{li}}\left(R_{\mathrm{c}}\right) g\left(R_{\mathrm{c}}\right) y_{12}^{\mathrm{so}}\left(R_{\mathrm{c}}\right) \\
& y_{62}^{\mathrm{so}}\left(R_{\mathrm{c}}\right)=-4 \pi G \rho^{\mathrm{li}}\left(R_{\mathrm{c}}\right) y_{12}^{\mathrm{so}}\left(R_{\mathrm{c}}\right)
\end{array}
$$

set 3: $\quad y_{33}^{\mathrm{so}}\left(R_{\mathrm{c}}\right)=1$,

the superscripts so and li refer to values in the solid mantle and in the liquid core, respectively. If a boundary value for $y_{i j}^{\text {so }}$ is not explicit given it has the value zero. It is $y_{i}=\sum_{j=1}^{3} Q_{j}^{\mathrm{so}} y_{i j}^{\mathrm{so}}$ in the solid mantel and the index $j$ stands for the different independent solutions. The $Q$ 's are determined by the boundary conditions at the surface, Eqs. (3.13).

The radial functions at the surface define the so called Love numbers

$$
\begin{aligned}
& h_{l}\left(\Omega_{l}^{m}\right)=y_{1}\left(R_{\mathrm{p}}, \Omega_{l}^{m}\right) g\left(R_{\mathrm{p}}\right), \\
& k_{l}\left(\Omega_{l}^{m}\right)=y_{5}\left(R_{\mathrm{p}}, \Omega_{l}^{m}\right)-1, \\
& \ell_{l}\left(\Omega_{l}^{m}\right)=y_{3}\left(R_{\mathrm{p}}, \Omega_{l}^{m}\right) g\left(R_{\mathrm{p}}\right),
\end{aligned}
$$

$h_{l}$ is the ratio of the height of the solid body tide to that of the deforming potential. $k_{l}$ is the ratio of the additional tidal potential, produced by the redistribution of mass, to the deforming potential. $\ell_{l}$ is the ratio of horizontal displacement of the crust to that of the equilibrium tide if the planet were fluid.

As already stated, planetary interiors for typical tidal forcing periods behave like viscoelastic bodies, which is modeled in this work by the Maxwell model. In the Maxwell model the stress strain relation is written in Cartesian coordinates as

$$
\dot{\sigma}_{i j}+(\mu / \eta)\left(\sigma_{i j}-\frac{1}{3} \sigma_{k k} \delta_{i j}\right)=2 \mu \dot{\epsilon}_{i j}+\left(K_{\mathrm{E}}-\frac{2}{3} \mu\right) \dot{\epsilon}_{k k} \delta_{i j}
$$

in this formula and in the following the Einstein notation is used. The Fourier transform 
of Eq. (3.22) leads to a Hooke like law

$$
\tilde{\sigma}_{i j}=2 \tilde{\mu}\left(\Omega_{l}^{m}\right) \tilde{\epsilon}_{i j}+\left[K_{\mathrm{E}}-\frac{2}{3} \tilde{\mu}\left(\Omega_{l}^{m}\right)\right] \tilde{\epsilon}_{k k} \delta_{i j}
$$

with

$$
\tilde{\mu}\left(\Omega_{l}^{m}\right)=\frac{\left(\Omega_{l}^{m}\right)^{2} \mu \eta^{2}}{\mu^{2}+\left(\Omega_{l}^{m}\right)^{2} \eta^{2}}+\mathrm{i} \frac{\mu^{2} \Omega_{l}^{m} \eta}{\mu^{2}+\left(\Omega_{l}^{m}\right)^{2} \eta^{2}},
$$

the tilde indicates complex values in the frequency domain. In an incompressible medium the dilatation $\nabla \cdot \boldsymbol{\xi}=\epsilon_{k k}$, with $\boldsymbol{\xi}$ the displacement vector, goes to zero while $K_{\mathrm{E}}$ goes to infinity in such a way that their product has a finite limit (Love, 1911), i.e.

$$
\lim _{K_{\mathrm{E}} \rightarrow \infty, \epsilon_{k k} \rightarrow 0} K_{\mathrm{E}} \epsilon_{k k}=\Pi
$$

Therefore under the condition of incompressibility Eq. (3.23) becomes

$$
\tilde{\sigma}_{i j}=2 \tilde{\mu}\left(\Omega_{l}^{m}\right) \tilde{\epsilon}_{i j}+\Pi \delta_{i j}
$$

According to the correspondence principle (Biot, 1954), the elastic problem can be extended to an equivalent viscoelastic problem, by simply replacing the parameter $\mu$ in Eq. (3.11) with the complex counterpart Eq. (3.24), this results in complex $y_{i}$ and complex love numbers. $\Omega_{l}^{m}$ can be replaced with $\Omega_{\mathrm{p}}$ because for tidal forcing periods of a few days, one can assume that the response frequency of the body equals the forcing frequency $\Omega_{\mathrm{p}}$.

Relevant for the calculation here is also the radial distribution of the tidal dissipation, according to Tobie et al. (2005) this is given by

$$
H_{\text {tide }}(r)=\frac{-21}{10} \frac{\Omega^{5} R_{\mathrm{p}}^{4} e^{2}}{r^{2}} S_{\tilde{\mu}} \Im(\tilde{\mu}),
$$

with

$$
\begin{aligned}
S_{\tilde{\mu}}= & \frac{5}{3}\left|2 \tilde{y}_{1}-l(l+1) \tilde{y}_{3}\right|^{2}-\frac{4}{3} r \mathfrak{R e}\left\{\frac{\mathrm{d} \tilde{y}_{1}^{\dagger}}{\mathrm{d} r}\left[2 \tilde{y}_{1}-l(l+1) \tilde{y}_{3}\right]\right\} \\
& +l(l+1) r^{2}\left|\tilde{y}_{4}\right|^{2} /|\tilde{\mu}|^{2}+l\left(l^{2}-1\right)(l+2)\left|\tilde{y}_{3}\right|^{2},
\end{aligned}
$$

the radial sensitivity to the shear modulus $\mu$, whereby this is the incompressible counterpart of Eq. (37) in Tobie et al. (2005). The superscript $\dagger$ stands for complex conjugated. 


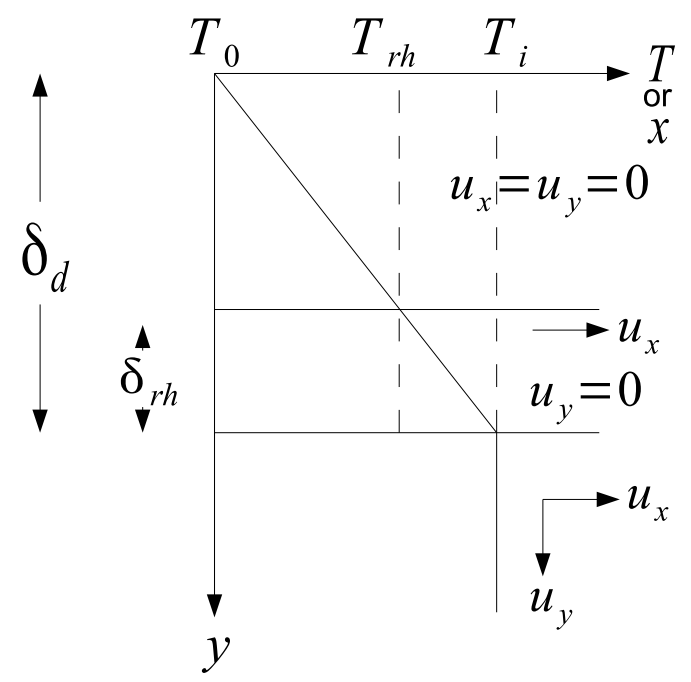

Figure 3.1.: Only the rheological boundary layer, with thickness $\delta_{\text {rh }}$, of the diffusive lid, with thickness $\delta_{\mathrm{d}}$, participates in the convective flow and only this part is mobile, $u_{x} \neq 0$. But also in the rheological boundary layer $u_{y}=0$, such that also across this layer heat is transported only by diffusion. Figure after Douce (2011).

\subsubsection{Heat transport mechanism}

\section{Convection}

The two extremes of convection regarding the efficiency were considered in this study, stagnant lid convection and plate tectonic convection. Stagnant lid convection is less efficient than plate tectonic convection. Viscosity is highly dependent on temperature, therefore if the surface temperatures are relatively cold, there is a highly viscous zone near the surface. In stagnant lid convection which e.g. occurs in Io, this highly viscous zone doesn't take part in the convection and through this region heat can generally only be transported by conduction (an exception will be shown below, the heat pipe model). Also on Earth the surface temperatures are relatively cool, but here the plate is broken into pieces because much of these are subductil (the oceanic plates), they can take part in the convection. Plate tectonic convection is therefore as efficient as isoviscous convection. In stagnant lid convection, convection is driven by the rheological boundary layer (see Fig. 3.1), this can become negative buoyant and sink, analogue to the plates in plate tectonic convection. Various numerical studies disagree if plate tectonic convection should become more likely with growing object size. A recent study (Stamenkovic and Breuer, 2014) suggests that with growing mass and inner temperature plate tectonic convection becomes less likely. 
The laws determining the heat flux in a planet are a matter of ongoing research. Here, we present a few relations which have been used in the past in similar studies of tidal dissipation, at the end of this section a few explanations will be given. For time dependent stagnant lid convection according to a numerical calculation in a spherical shell with internal heating and free slip boundary conditions (Reese et al., 2005)

$$
N u=0.67 \theta^{-4 / 3} R a_{\mathrm{i}}^{1 / 3} .
$$

With the Nusselt number

$$
N u=\frac{F d_{\mathrm{c}}}{k\left(T_{\mathrm{i}}-T_{\mathrm{s}}\right)},
$$

Rayleigh number

$$
R a_{\mathrm{i}}=\frac{\alpha \rho_{\mathrm{m}} g_{\mathrm{s}}\left(T_{\mathrm{i}}-T_{\mathrm{s}}\right) d_{\mathrm{c}}^{3}}{\kappa \eta\left(T_{\mathrm{i}}\right)},
$$

and the Frank-Kamenetskii parameter $\theta=\gamma\left(T_{\mathrm{i}}-T_{\mathrm{S}}\right)$, for a strictly temperature dependent viscosity $\gamma=E_{a} /\left(\bar{R} T_{\mathrm{i}}^{2}\right)$ (Reese et al., 1999; Moore, 2003), $F$ is the heat flux, $d_{\mathrm{c}}$ the thickness of the convecting layer without the stagnant lid (in the four layer model $d_{\mathrm{c}}$ equals the thickness of the asthenosphere) and $T_{\mathrm{i}}$ is the interior temperature. For the case of stagnant lid convection $T_{\mathrm{i}}=T_{\mathrm{L}}+a_{\mathrm{rh}} \gamma^{-1}$, with $T_{\mathrm{L}}$ the temperature at the bottom of the lid, and $a_{\mathrm{rh}} \gamma^{-1}$ the temperature jump across the rheological boundary layer, where $a_{\text {rh }}$ a numerical coefficient, for the spherical shell $a_{\mathrm{rh}}=3.2$ (Reese et al., 1999). For the remaining constants see Table 3.1 .

For comparison, also another scaling for time dependent stagnant lid convection will be used. Recently a study by Deschamps and Lin (2014) proposed the scaling

$$
N u=1.46 \theta^{-1.21} R a_{\mathrm{i}}^{0.27} .
$$

It was obtained in a 3D-Cartesian box, with free slip conditions at the top and bottom boundary heated from below. It should be valid especially for the regime below $R a<$ $10^{8}$, so below the chaotic regime, for the chaotic regime the scaling with a $1 / 3$ Rayleigh exponent should be valid (Dumoulin et al., 1999).

For plate tectonic convection with internal heating a scaling as in O'Connell and Hager (1980) is used

$$
N u=\frac{2 a_{2}}{R a_{\mathrm{c}}^{1 / 4}} R a_{F}^{1 / 4} \approx 0.35 R a_{F}^{1 / 4},
$$

with a flow geometry constant $a_{2} \sim 1$ and the critical Rayleigh number $R a_{\mathrm{c}} \sim 1100$ and

$$
R a_{F}=\frac{\alpha \rho g F d_{\mathrm{c}}^{4}}{k \kappa \eta\left(T_{\mathrm{i}}\right)}
$$


Because $R a_{F} / R a_{\mathrm{i}}=N u$ the Eq. (3.33) is equivalent to

$$
N u=\frac{\left(2 a_{2}\right)^{4 / 3}}{R a_{\mathrm{c}}^{1 / 3}} R a_{\mathrm{i}}^{1 / 3} \approx 0.244 R a_{\mathrm{i}}^{1 / 3}
$$

The correctness of this scaling is supported by newer numerical simulations by Wolstencroft et al. (2009), who find scaling relations which differ only slightly.

It is assumed that the scaling relations for $N u(R a)$ used here with a Rayleigh number exponent of $1 / 3$ are also applicable for convection in a material with a high melt fraction and high Rayleigh number because this scaling is in accordance with boundary layer theory (Turcotte and Schubert, 2002; Douce, 2011) and generally numerical simulations at high Rayleigh number differ only little from this scaling, see the review by Ahlers et al. (2009) and references therein.

Now the case of stagnant lid convection and the difference to plate tectonic convection will be elucidated according to the work in Douce (2011). As already stated stagnant lid convection is driven by the temperature difference across the rheological boundary layer $\Delta T_{\mathrm{rh}}=T_{\mathrm{i}}-T_{\mathrm{rh}}$, with $T_{\mathrm{rh}}$ the temperature at the top of the rheological boundary layer (see Figure 3.1). Convection with moving plates is driven by the full temperature difference $\Delta T=T_{\mathrm{i}}-T_{\mathrm{S}}$, with $T_{\mathrm{S}}$ the surface temperature. The rheological boundary layer can be defined as the part of the thermal boundary layer in which the viscosity $\eta_{\mathrm{rh}}$ is of the same order of magnitude as the viscosity of the convecting fluid $\eta_{\mathrm{i}}=\eta\left(T_{\mathrm{i}}\right)$. For the viscosity an Arrhenius law is used

$$
\eta=K_{\mathrm{a}} e^{E_{\mathrm{a}} /(\bar{R} T)}
$$

with $K_{\mathrm{a}}$ a material property. Restriction of temperature to a relatively narrow interval allows us to write the viscosity as

$$
\eta=\eta_{0} e^{\frac{E_{\mathrm{a}}}{R} \frac{T_{0}-T}{T T_{0}}}
$$

with $T_{0}$ a reference temperature in this interval and the viscosity $\eta_{0}$ at this reference temperature. If $T$ and $T_{0}$ are not too different, the approximation

$$
\eta \approx \eta_{0} e^{-\gamma\left(T-T_{0}\right)}
$$

is valid. With $T_{0}=T_{\mathrm{i}}$ it is

$$
\eta_{\mathrm{rh}} \approx \eta_{\mathrm{i}} \mathrm{e}^{\gamma \Delta T_{\mathrm{rh}}},
$$


the condition $\eta_{\mathrm{rh}} \sim \eta_{\mathrm{i}}$ is satisfied if $\gamma \Delta T_{\mathrm{rh}}=1$. It is therefore

$$
\Delta T_{\mathrm{rh}}=\frac{1}{\gamma}
$$

The Rayleigh number based on the temperature $\gamma^{-1}$ is

$$
R a_{\mathrm{e}}=\frac{\alpha \rho g d_{\mathrm{c}}^{3}}{\kappa \eta_{\mathrm{i}} \gamma}=R a_{\mathrm{i}}(\gamma \Delta T)^{-1}
$$

Assuming now convection beneath the lid is similar to constant viscosity convection with a driving temperature $\gamma^{-1}$, the scaling for plate tectonic convection can be used to estimate the scaling for stagnant lid convection by simply substituting $\Delta T$ by $\Delta T_{\mathrm{rh}}$. Therefore, according to Eq. (3.35) the heat flow is given by

$$
F \approx 0.244 R a_{\mathrm{e}}^{1 / 3} \frac{k \Delta T_{\mathrm{rh}}}{d_{c}} .
$$

The Nusselt number is still defined on basis of the full temperature difference and therefore according to Eq. (3.35)

$$
N u=\frac{F}{\frac{k \Delta T}{d_{c}}}=\frac{\left(2 a_{2}\right)^{4 / 3}}{R a_{\mathrm{c}}^{1 / 3}} R a_{\mathrm{e}}^{1 / 3}(\gamma \Delta T)^{-1} \approx 0.244 R a_{\mathrm{i}}^{1 / 3}(\gamma \Delta T)^{-4 / 3},
$$

which is on the order of the scaling according to Eq. (3.29) and only differs by the prefactor.

\section{Melt migration}

In a partial molten mantle, magma transport can be important for the heat transport, as for example stated by Moore (2001) for Io's asthenosphere. The governing equations for heat transport by melt through the mantle (Moore, 2001) are:

- The conservation of mass

$$
\nabla \cdot\left(\mathbf{v}_{\mathrm{l}} \phi\right)=\frac{\Gamma}{\rho_{\mathrm{l}}} \text { and } \nabla \cdot\left(\mathbf{v}_{\mathrm{s}}(1-\phi)\right)=-\frac{\Gamma}{\rho_{\mathrm{s}}}
$$

with $\mathbf{v}_{\mathrm{l}}$ and $\mathbf{v}_{\mathrm{s}}$ the velocities of the liquid and solid components, $\rho_{\mathrm{l}}$ and $\rho_{\mathrm{s}}$ the liquid and solid densities, $\phi$ the melt volume fraction, and $\Gamma$ the melt production rate (in $\mathrm{kg} \mathrm{m}^{-3} \mathrm{~s}^{-1}$ ). Contrary to Moore (2001) we take into account the difference between the densities of liquid and solid in Eq. (3.44). 
- The Darcy flow equation

$$
\phi\left(\mathbf{v}_{\mathrm{l}}-\mathbf{v}_{\mathrm{s}}\right)=\frac{k_{\phi} \Delta \rho \mathbf{g}}{\eta_{1}}
$$

with the permeability $k_{\phi}=b^{2} \phi^{n_{\mathrm{m}}} / \tau, b$ is a typical grain size, $\tau$ is a constant which depends on the exponent $n_{\mathrm{m}}, \eta_{\mathrm{l}}$ is the viscosity of the liquid and $\Delta \rho$ the density contrast between liquid and solid material. In our calculations we chose $n_{\mathrm{m}}=2.6$ and $\tau=60$ (Miller et al., 2014). These expressions are valid as long as the solid matrix does not disaggregate and the melt does form an interconnected network.

- The conservation of energy

$$
\Xi \Gamma=H
$$

with $\Xi$ the latent heat of fusion and $H$ the heating rate per volume.

The system of Eqs. (3.44)-(3.46) can be written as two first order equations for $\phi$ and $v_{1}$ by eliminating $\Gamma$ and $v_{\mathrm{s}}$

$$
\begin{gathered}
\frac{d v_{\mathrm{l}}}{d r}=\frac{\Gamma}{\phi \rho_{l}}-v_{\mathrm{l}}(r)\left(\frac{2}{r}+\frac{1}{\phi} \frac{d \phi}{d r}\right), \\
\frac{d \phi}{d r}=\frac{\Delta \rho \phi^{2}\left(2 b^{2} g \rho_{\mathrm{l}} \rho_{\mathrm{s}}\left(\phi^{n_{\mathrm{m}}}-\phi^{n_{\mathrm{m}}-1}\right)-\Gamma \eta \tau r\right)+\phi \eta \tau \rho_{\mathrm{S}} \Gamma r}{\rho_{\mathrm{l}} \rho_{\mathrm{s}} r\left(b^{2} g \phi^{n_{\mathrm{m}}} \Delta \rho\left(n_{\mathrm{m}}-1-\phi n_{\mathrm{m}}\right)+\phi \eta \tau v_{\mathrm{l}}\right)} .
\end{gathered}
$$

From Eq. (3.44) we can deduce

$$
\rho_{\mathrm{l}} \mathbf{v}_{\mathrm{l}} \phi+\rho_{\mathrm{s}} \mathbf{v}_{\mathrm{s}}(1-\phi)=0
$$

this equation is valid globally. If one considers parts of the melting zone, one has to consider flux of mass into or out of the zone, for instance due to an upwelling mantle. Equations (3.45)-(3.49) can be rearranged such that the heat flux due to melt migration $F_{M}$ is given by

$$
F_{M}=\frac{\int H \cdot d V}{4 \pi R_{\mathrm{p}}^{2}}=\rho_{\mathrm{l}} L\left|\mathbf{v}_{\mathrm{l}}\right| \phi=\rho_{\mathrm{l}} L \frac{1-\phi}{\phi\left(\rho_{\mathrm{l}} / \rho_{\mathrm{s}}-1\right)+1} \frac{k_{\phi} \Delta \rho|\mathbf{g}|}{\eta_{\mathrm{l}}} .
$$

For $|\mathbf{g}|$ we use the surface gravity. $\Delta \rho=\rho_{\mathrm{s}}-\rho_{\mathrm{l}}$ and $\eta_{\mathrm{l}}$ are calculated with the MELTS program (Ghiorso and Sack, 1995) and for $\rho_{\mathrm{s}}$ we use the mantle density $\rho_{\mathrm{m}}$. The radial component of the liquid and solid velocities is approximated through the absolute value of $v_{\mathrm{l}}$ and $v_{\mathrm{s}}$, respectively. Equation (3.50) is only correct if the melting zone has a constant melt volume fraction. This would roughly be the case for large enough grain sizes. We checked the validity of this approach by solving the six coupled ODEs (Eq. (3.11)) with a fixed viscosity and shear modulus in the deep mantle and the lithosphere, but in the asthenosphere the viscosity was determined by solving additionally Eqs. (3.47) and (3.48) with initial conditions $\phi=0.01$ and $v_{\mathrm{l}}=0$ at the base of the molten zone. With the 
obtained value for $\phi$ and the resulting temperature the viscosity can be determined selfconsistently and becomes thereby depth dependent in the asthenosphere. The heating rate per volume is given by Eq. (3.27). In the asthenosphere, the system of ODEs (Eqs. (3.11), (3.47) and (3.48)) can not be solved according to the scheme described in Sect. 3.1.1, because the equations are now nonlinear. We therefore use a shooting method as described in Sect. 2.2, but shooting to a fitting point is this time not necessary. Again a Runge Kutta Cash-Karp method of fifth order with an adaptive stepsize control was used to integrate the ODEs.

\section{The heat pipe model}

In stagnant lid convection one generally assumes that through the lithosphere (the lid) heat can only be transported by conduction. This resulted in problems regarding Io with a high heat flow and high mountains on it because high heat flow results in a small lithosphere which is contradictory with high mountains. An explanation was given by O'Reilly and Davies (1981), the heat pipe model. They assumed that part of the heat is removed by advection through pipes in the lithosphere.

\subsubsection{Coupling between heat transport and tidal heat production}

Tidal dissipation can be estimated by the determination of a balance between heat transport and tidal heat production rate. Therefore the tidal dissipation depends on the efficiency of the heat transport mechanism.

The convective heat flux is given by a relation of the form

$$
F_{\text {conv }}=a_{\mathrm{c}} \frac{k\left(T_{\mathrm{i}}-T_{\mathrm{s}}\right)}{d_{\mathrm{c}}} \theta^{-\alpha} R a_{\mathrm{i}}^{\varsigma},
$$

where $a_{\mathrm{c}}, \alpha$, and $\varsigma$ are constants depending on the $N u\left(R a_{\mathrm{i}}\right)$ relation of choice. This flux must equal the tidal heat flux through the surface

$$
F_{\text {tidal }}=\frac{D_{\mathrm{T}}}{4 \pi r_{1}^{2}}
$$

For the four layer model of Io we calculate with a fixed $r_{1}=R_{\mathrm{p}}-d_{\text {lit }}$, with $d_{\text {lit }}$ the lithosphere thickness (see Table 3.4). For plate tectonic convection we approximate $r_{1}$ by $R_{\mathrm{p}}$. For these cases, equating Eqs. (3.51) and (3.52) fixes $T_{\mathrm{i}}$ if $T_{\mathrm{s}}$ is given so that $D_{\mathrm{T}}$ is computable. For stagnant lid convection in the homogeneous mantle model (this is a two 
layer model with a liquid core and a solid mantle) we calculate $r_{1}$ by taking into account the thermal effect of the lithosphere, as done by Moore (2003). In such a model, the upper surface of the convection zone is the bottom of the lid, which is located at radius $r_{1}$ and has temperature $T_{1}$, so that one has to find conditions to determine $r_{1}, T_{1}$ and $T_{\mathrm{i}}$. These are the balance between tidal and convective heat fluxes and two expressions for $T_{1}$ (Reese et al., 1999)

$$
T_{1}=T_{\mathrm{i}}-a_{\mathrm{rh}} \frac{1}{\gamma}=T_{\mathrm{s}}+\frac{H}{6 k}\left(R_{\mathrm{p}}^{2}-r_{1}^{2}\right)+\left(\frac{F_{\mathrm{conv}} r_{1}^{2}}{k}-\frac{H r_{1}^{3}}{3 k}\right)\left(\frac{1}{r_{1}}-\frac{1}{R_{\mathrm{p}}}\right),
$$

with

$$
H=\frac{D_{\mathrm{T}}}{(4 / 3) \pi\left(r_{1}^{3}-\left(r_{1}-d_{\mathrm{c}}\right)^{3}\right)},
$$

being the rate of internal heat generation (in $\mathrm{W} / \mathrm{m}^{3}$ ). If we consider only melt migration the heat flux is $F_{M}$ instead of $F_{\text {conv }}$ and if we consider both it is $F_{M}+F_{\text {conv }}$ instead of $F_{\text {conv }}$.

In the study of Io below, $T_{\mathrm{s}}$ is assumed given by observations. In the study of exoplanets, $T_{\mathrm{S}}$ is determined from the tidal dissipation and the irradiance of the host star (Henning et al., 2009)

$$
T_{\mathrm{s}}=\left(\frac{D_{\mathrm{T}}}{4 \pi R_{\mathrm{p}}^{2} \sigma_{\mathrm{sb}} \epsilon_{\mathrm{r}}}+\frac{1-A}{4 \sigma_{\mathrm{sb}} \epsilon_{\mathrm{r}}}\left(\frac{L_{\mathrm{star}}}{4 \pi a^{2}}\right)\right)^{1 / 4}
$$

$\sigma_{\mathrm{sb}}$ is the Stefan-Boltzmann constant, $\epsilon_{\mathrm{r}}$ the emissivity, $A$ the albedo, $L$ the stellar luminosity, and $a$ the semi major axis. Finally, the temperature $T$ in $D_{\mathrm{T}}(T)$ is identified with $T_{\mathrm{i}}$. 


\subsubsection{Rheology}

The rheology used for this study is similar as in Moore (2003). For the temperature dependence of the shear modulus and viscosity, it is

$$
\begin{aligned}
\mu(T)= \begin{cases}\mu_{\text {sol }}, & \text { if } T \leq T_{\text {crit }} \\
10^{\mu_{1} / T+\mu_{2}} \mathrm{~Pa}, & \text { if } T_{\text {crit }}<T \leq T_{\text {dis }} \\
0 \mathrm{~Pa}, & \text { if } T_{\text {dis }}<T\end{cases} \\
\eta(T)= \begin{cases}\eta_{0} \cdot \mathrm{e}^{E_{A} / \bar{R} T}, & \text { if } T \leq T_{\text {sol }} \\
\eta_{0} \cdot \mathrm{e}^{E_{A} / \bar{R} T} \mathrm{e}^{-B \phi}, & \text { if } T_{\text {sol }}<T \leq T_{\text {dis }} \\
(1.35 \phi-0.35)^{-5 / 2} \cdot 10^{3} \mathrm{Pas}, & \text { if } T_{\text {dis }}<T,\end{cases}
\end{aligned}
$$

$B$ is a dimensionless melt fraction coefficient. In this study $B=25$ and $B=40$ are used. We chose $B=25$ because Mei et al. (2002) reported this value for diffusion creep (see also Karato (2013)) and $B=40$ because it is the highest value that Moore (2003) used. As in Moore (2003) the solidus temperature $T_{\text {sol }}$ is assumed to be at $1598 \mathrm{~K}$ and the critical temperature $T_{\text {crit }}$ at $1600 \mathrm{~K}$. $\phi$ is the volume melt percentage. It increases linearly from the solidus to the liquidus at a rate of $1 \%$ per Kelvin. The liquidus is assumed to be at $1698 \mathrm{~K}$. Above the disaggregation temperature $T_{\text {dis }}$ the shear modulus is zero. Disaggregation happens at $\sim 30 \%$ partial melting (Moore, 2001; Scott and Kohlstedt, 2006). The figures in this study are partly plotted up to $1670 \mathrm{~K}$, which corresponds to a melt fraction of $72 \%$. Keep in mind that the solid matrix may already have disaggregated at $\sim 1630 \mathrm{~K}$. Values for the parameters can be found in Table 3.1 and Table 3.3. Viscosity and shear modulus as a function of temperature are plotted in Fig. 3.2. The bulk modulus can be calculated
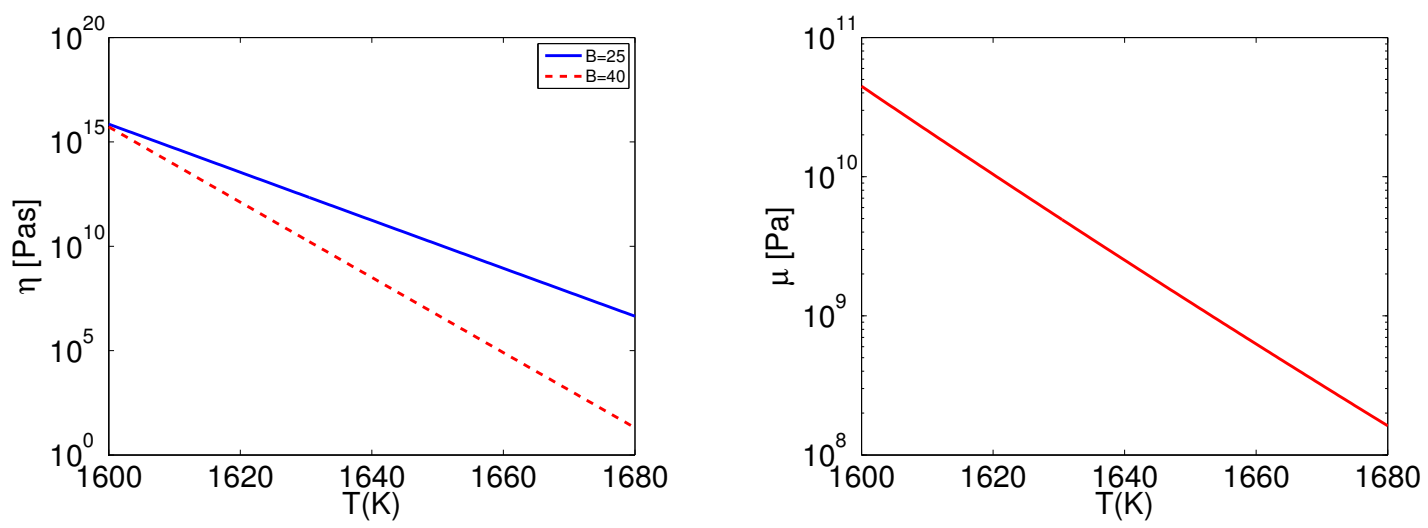

Figure 3.2.: Left: Viscosity as a function of temperature; Right: Shear modulus as a function of temperature 
according to

$$
K_{\mathrm{E}}=\rho V_{\mathrm{p}}^{2}-\frac{4}{3} \mu
$$

where $v_{\mathrm{p}}$ is the velocity of the elastic p-wave.

\subsubsection{Tidal quality factor}

We calculate the tidal quality factor according to Murray and Dermott (1999)

$$
Q=\frac{\Omega_{\mathrm{p}} E_{0}}{D_{\mathrm{T}}}
$$

with $E_{0}$ the peak energy stored during the cycle

$$
E_{0}=\frac{21}{2} \frac{\left|k_{2}\right| \Omega_{\mathrm{p}}^{4} R_{\mathrm{p}}^{5} e^{2}}{G} .
$$

With Eq. (3.3) this gives

$$
Q=-\frac{\left|k_{2}\right|}{\Im\left(k_{2}\right)} .
$$

The modified tidal quality factor is defined as

$$
Q^{\prime}=\frac{3 Q}{2\left|k_{2}\right|}=-\frac{3}{2 \Im\left(k_{2}\right)}
$$

The modified tidal quality factor is defined such that it only depends on $\Im\left(k_{2}\right)$, which is the quantity that appears in Eq. (3.3) and that for a homogeneous liquid sphere $\left(k_{2}=3 / 2\right)$ $Q^{\prime}=Q$.

\subsection{Validation}

The validation of the code computing $\Im\left(k_{2}\right)$ through a solution of coupled ODEs (see Sect. 3.1.1) was done by a comparison with various other studies, in particular with Segatz et al. (1988) and Sotin et al. (2009). The results from the comparison with Segatz et al. (1988) and Sotin et al. (2009) are displayed here. As written by Beuthe (2013) Segatz et al. (1988) forgot a factor $2 \pi$ in the angular frequency. Thus all viscosity values in Segatz et al. (1988) should be divided by $2 \pi$. Therefore the viscosity values here are adjusted through a division by $2 \pi$ for this validation. The results with our code are shown in Fig. 3.3(a) and 3.3(b). The figures agree relatively well with Fig. 5 of Segatz et al. (1988). The calculation for 3.3(b) was performed as in Tobie et al. (2005), that means with a finite bulk 

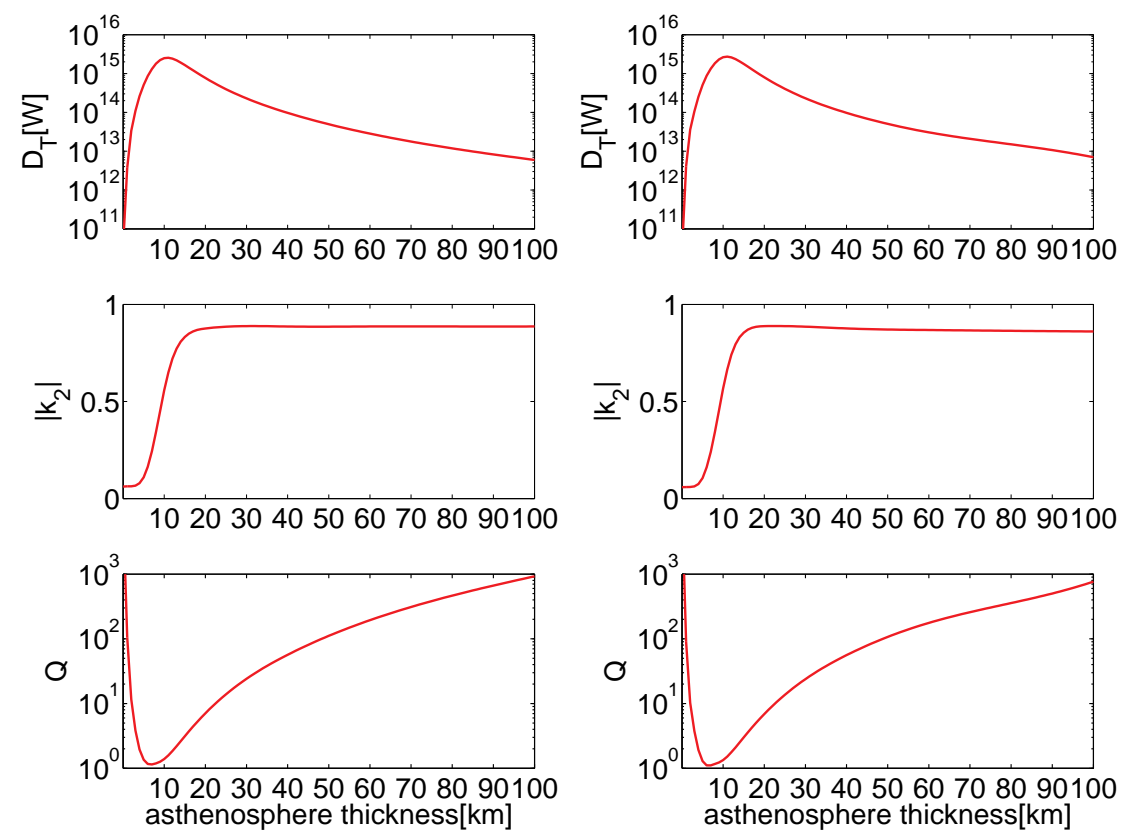

(a)

(b)

Figure 3.3.: Tidal dissipation rate $D_{\mathrm{T}}$, modulus of the tidal love number $k_{2}$ and dissipation factor $\mathrm{Q}$ versus asthenosphere thickness of Io, using parameters from Segatz et al. (1988), and equivalent to their Fig. 5. (a): own incompressible calculations; (b): own compressible calculations.

modulus $K_{\mathrm{E}}$ and taking into account the inertial term. We choose $v_{\mathrm{p}}=6000 \mathrm{~ms}^{-1}$ for the core and $v_{\mathrm{p}}=8000 \mathrm{~ms}^{-1}$ for the silicate mantle (Tobie et al., 2005). For simplification the results in the following section were obtained with the approximation of incompressibility and neglecting the inertial term. The comparison with Sotin et al. (2009) is shown in Fig. 3.4 .

\subsection{Results}

\subsubsection{Tidal dissipation of lo}

An estimate of the tidal dissipation for Io has already been made by Moore (2003), their coupled tidal convection model led to a heat flux of $\approx 2 \times 10^{13} \mathrm{~W}$. This is lower as the observed value $0.6-1.6 \times 10^{14} \mathrm{~W}$ (Moore et al., 2007). Therefore they deduced that either Io is currently out of thermal equilibrium or another heat transport mechanism such as melt segregation determines Io's thermal state. In Lainey et al. (2009) it was shown that Io is close to thermal equilibrium, according to this presumably only another heat transport mechanism would be an explanation. 


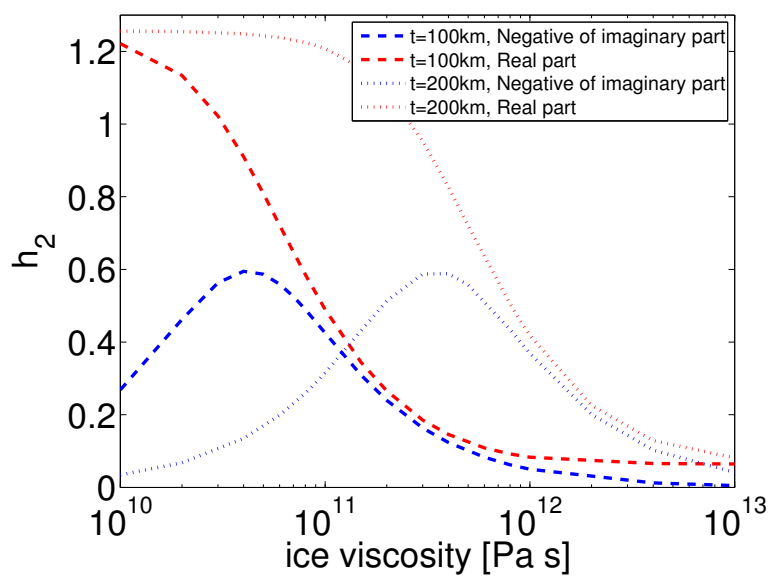

Figure 3.4.: Love number $h_{2}$ versus ice viscosity of Europa using parameters from Sotin et al. (2009), and equivalent to their Fig. 5.

\begin{tabular}{ccc}
\hline \hline Symbol & Description & Value \\
\hline$c_{\mathrm{p}}$ & Specific heat at constant pressure & $10^{3} \mathrm{Jkg}^{-1} \mathrm{~K}^{-1 \mathrm{a}}$ \\
$E_{A}$ & Activation energy for subsolidus mantle creep & $3.33 \times 10^{5} \mathrm{Jmole}^{-1}$ \\
$G$ & Gravitational constant & $6.67 \times 10^{-11} \mathrm{~m}^{3} \mathrm{~kg}^{-2} \mathrm{~s}^{-2}$ \\
$k$ & Thermal conductivity & $4.0 \mathrm{Wm}^{-1} \mathrm{~K}^{-1 \mathrm{~b}}$ \\
$\alpha$ & Thermal expansion coefficient & $3.0 \times 10^{-5} \mathrm{~K}^{-1 \mathrm{~b}}$ \\
$\kappa$ & Thermal diffusivity & $10^{-6} \mathrm{~m}^{2} \mathrm{~s}^{-1 \mathrm{~b}}$ \\
$\bar{R}$ & Universal gas constant & $8.32 \mathrm{JK}^{-1} \mathrm{~mole}^{-1}$ \\
$\Xi$ & Latent heat & $5 \times 10^{5} \mathrm{Jkg}^{-1 \mathrm{c}}$ \\
$T_{\text {sol }}$ & Solidus temperature & $1598 \mathrm{~K}^{\mathrm{b}}$ \\
$T_{\text {crit }}$ & Critical temperature & $1600 \mathrm{~K}^{\mathrm{b}}$ \\
$T_{\mathrm{dis}}$ & Dissagregation temperature & not fixed \\
$\sigma_{s b}$ & Stefan Boltzmann constant & $5.67 \times 10^{-8} \mathrm{Wm}^{-2} \mathrm{~K}^{-4}$ \\
$\epsilon_{r}$ & Emissivity & $1^{\mathrm{d}}$ \\
\hline
\end{tabular}

Table 3.1.: Notation and Parameters which are treated as constants in this study.

References. $\quad{ }^{a}$ Fischer and Spohn (1990); $\quad{ }^{b}$ Moore (2003); $\quad{ }^{c}$ Moore (2001); d Henning et al. (2009)

\begin{tabular}{ccc}
\hline \hline Symbol & Description & Value \\
\hline$e$ & Orbital eccentricity & $0.0041^{\mathrm{a}}$ \\
$m[\mathrm{~kg}]$ & Mass of the object & $8.93 \times 10^{22 \mathrm{~b}}$ \\
$R_{\mathrm{p}}[\mathrm{m}]$ & Radius of the object & $1.82 \times 10^{6 \mathrm{~b}}$ \\
$T_{\mathrm{S}}[\mathrm{K}]$ & Surface temperature & $100^{\mathrm{c}}$ \\
$R_{\mathrm{c}}[\mathrm{m}]$ & Core radius & $9.80 \times 10^{5 \mathrm{~d}}$ \\
$\Omega_{\mathrm{p}}\left[\mathrm{s}^{-1}\right]$ & Orbital angular velocity & $4.11 \times 10^{-5 \mathrm{a}}$ \\
$\rho_{\mathrm{m}}\left[\mathrm{kgm}^{-3}\right]$ & Mantle density & $3300^{\mathrm{d}}$ \\
\hline
\end{tabular}

Table 3.2.: Notation and parameters for Io.

References. $\quad{ }^{a}$ Jacobson (2003); $\quad{ }^{\mathrm{b}}$ Schubert et al. (2004); $\quad{ }^{\mathrm{c}}$ Rathbun et al. (2004); d Segatz et al. (1988) 


\begin{tabular}{cc}
\hline \hline Symbol & Value \\
\hline$\mu_{\text {sol }}$ & $5.0 \times 10^{10} \mathrm{~Pa}$ \\
$\mu_{1}$ & $8.2 \times 10^{4} \mathrm{~K}$ \\
$\mu_{2}$ & -40.6 \\
$\eta_{0}$ & $1.6 \times 10^{5} \mathrm{Pas}$ \\
\hline
\end{tabular}

Table 3.3.: Parameter values for the rheology model according to Fischer and Spohn (1990).

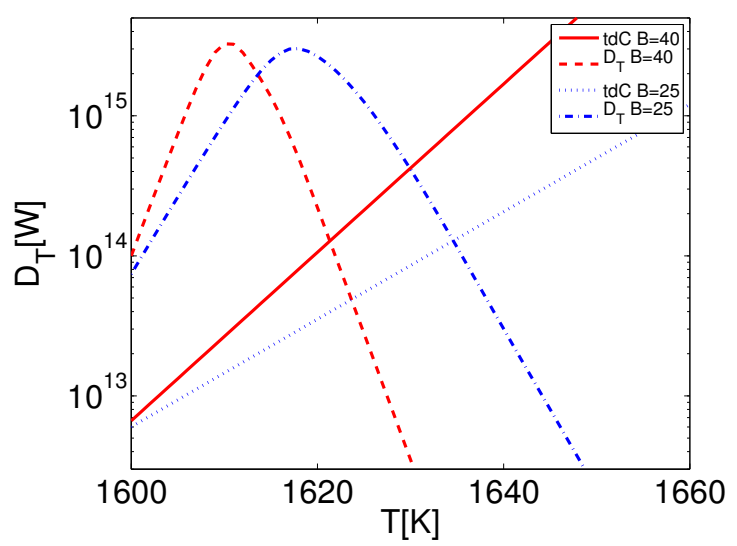

Figure 3.5.: Dissipation according to time dependent convection (tdC) and tidal heat $\left(\mathrm{D}_{\mathrm{T}}\right)$ for two different rheologies in a homogenous mantle model. Model parameter see Table 3.1-3.3. $N u(R a)$ according to Eq. (3.29). Credit: Clausen \& Tilgner, A\&A, 2015, DOI: 10.1051/0004$6361 / 201526082$, reproduced with permission OESO.

We will now use a $N u(R a)$ scaling according to Eq. (3.29) for the homogeneous mantle model to determine the tidal dissipation in Io. With this scaling it is $D_{\mathrm{T}} \approx 1.1 \times 10^{14} \mathrm{~W}$, as can be deduced from Fig. 3.5. Therefore with this scaling the obtained dissipation rate is on the order of the observed value. In Clausen and Tilgner (2015) we wrote that we were unable to reproduce the results of Moore (2003), if we use the scaling for the heat transport which is stated in his work. Furthermore we wrote that the results in Moore (2003) were presumably obtained with the heat transfer law for steady-state convection. But it turns out that his model is more sophisticated than we originally thought. He used a multi-layered model and thereby determined the radially dependent viscosity and shear modulus to calculate the tidal dissipation in Io. So his model is not identical with our simple homogeneous mantle model. It is therefore not surprising that the results for the tidal dissipation differ, even if we use the same scaling for the heat transport. The confusion arose among other things by his Fig. 4, in which the tidal heating is plotted as a function of a single temperature. It is not clear to us what is meant by this temperature in a multi-layered model. He obtained a tidal dissipation of $\approx 2 \times 10^{13} \mathrm{~W}$ with his model. So our homogeneous mantle model may be oversimplified. 


\begin{tabular}{cccc}
\hline \hline Symbol & Description & M1 & M2 \\
\hline$d_{\mathrm{L}}[\mathrm{m}]$ & Lithosphere thickness & $30 \times 10^{3}$ & $30 \times 10^{3}$ \\
$\mu_{\mathrm{L}}[\mathrm{Pa}]$ & Lithosphere shear modulus & $65 \times 10^{9}$ & $65 \times 10^{9}$ \\
$\mu_{\mathrm{dm}}[\mathrm{Pa}]$ & Deep mantle shear modulus & $60 \times 10^{9}$ & $50 \times 10^{9}$ \\
$\eta_{\mathrm{L}}[\mathrm{Pas}]$ & Lithosphere viscosity & $10^{23}$ & $10^{23}$ \\
$\eta_{\mathrm{dm}}[\mathrm{Pas}]$ & Deep mantle viscosity & $10^{20}$ & $10^{16}$ \\
\hline
\end{tabular}

Table 3.4.: M1 values according to Segatz et al. (1988) and M2 with a lower shear modulus and viscosity in the deep mantle. For the lithosphere thickness see also Turtle et al. (2007).

Furthermore, at the interior temperature of the equilibrium found in Fig. 3.5, the rheological law assumes a nonzero melt fraction, so that some contribution of melt migration to the total heat transport is likely. This motivates us to study an asthenosphere model, in which Io consists of a liquid core, a deep mantle with zero melt fraction, an asthenosphere, and a lithosphere. We define the asthenosphere as the zone with a nonzero melt fraction. An asthenosphere with a rock melt fraction of $20 \%$ or more and at least $50 \mathrm{~km}$ thickness was recently inferred from magnetic data by Khurana et al. (2011), which supports this model. The results for different asthenosphere thicknesses are shown in Fig. 3.6(a). The behavior of the tidal dissipation as a function of the asthenosphere temperature $D_{\mathrm{T}}(T)$ depends sensitively on the asthenosphere thickness while the convective heat flux according to Eqs. (3.29) and (3.30) does not. This time we only plot the results for $B=25$ and not $B=40$, because the difference between the convection-dissipation equilibrium values for different $B$ values is negligible, at least for $25 \leq B \leq 40$, as made plausible in Fig. 3.5 and as written in Moore (2003).

Hereinafter we assume $10^{14} \mathrm{~W}$ as the nominal value of Io's tidal dissipation. Furthermore for the following it is important to know that our calculations have shown that with melt migration stable equilibra are also possible where the slope of the tidal heating curve is positive. At the temperature at which the tidal dissipation equals $10^{14} \mathrm{~W}$ the convective heat flux must be less than or equal to $10^{14} \mathrm{~W}$. If it is less than $10^{14} \mathrm{~W}$ a contribution by melt migration is possible. This requires an asthenosphere thickness of at least $100 \mathrm{~km}$ as seen in Fig. 3.6(a). An asthenosphere thinner than $100 \mathrm{~km}$ is also unlikely because it requires a presumably too high disaggregation temperature to obtain a tidal dissipation of $10^{14} \mathrm{~W}$. From Fig. 3.6(a) it can be deduced that if melt migration is the dominant mechanism (i.e. transporting more heat than convection) the asthenosphere must be bigger then $200 \mathrm{~km}$, but still roughly $50 \%$ of the heat is transported by convection to obtain a tidal dissipation of $10^{14} \mathrm{~W}$ for a $200 \mathrm{~km}$ thick asthenosphere.

In Fig. 3.6(b) a similar plot as in Fig. 3.6(a) is shown but with a thermal conductivity of $2 \mathrm{Wm}^{-1} \mathrm{~K}^{-1}$ and different asthenosphere thicknesses. Now the asthenosphere needs to 
be larger than $75 \mathrm{~km}$ and for melt migration as the dominant heat transport mechanism larger than $150 \mathrm{~km}$. For this conductivity and a $150 \mathrm{~km}$ thick asthenosphere roughly $50 \%$ of the heat is transported by convection to obtain a tidal dissipation of $10^{14} \mathrm{~W}$ before the peak. But $2 \mathrm{Wm}^{-1} \mathrm{~K}^{-1}$ is presumably a too low value which may not even be valid for familiar igneous rocks, such as basalt, at laboratory pressures (Stacey, 2007).

The result is also influenced by the right parameterization of convection, as written by Dumoulin et al. (1999) a $N u(R a)$ parameterization with a $1 / 3$ Rayleigh exponent is valid only for cases in the chaotic regime, this regime applies for large values of the Rayleigh number typically larger than $10^{8}$, which is the case for Io. But for comparison in Fig. 3.7(a) a $N u(R a)$ scaling according to Eq. (3.32) was used. Then the asthenosphere needs to be larger than $75 \mathrm{~km}$ and for melt migration as the dominant heat transport mechanism larger than $150 \mathrm{~km}$. In Fig. 3.7(b) a $N u(R a)$ scaling according to Eq. (3.29) was used but with $\varsigma=0.3$ and $\alpha=1.3$ instead of $1 / 3$ and $4 / 3$. For that case the asthenosphere needs to be thicker than $60 \mathrm{~km}$ and melt migration is the dominant heat transport already for an asthenosphere thicker than $100 \mathrm{~km}$. For comparison also a model with higher dissipation in the mantle was considered, see Fig. 3.6(a) the M2 model. For this model with an $100 \mathrm{~km}$ thick asthenosphere at $1631 \mathrm{~K}$ it is $D_{\mathrm{T}} \approx 10^{14} \mathrm{~W}$ and roughly half of the tidal heating is produced in the asthenosphere at this temperature. For the M1 model with a $100 \mathrm{~km}$ thick asthenosphere at $1634.4 \mathrm{~K}$ it is also $D_{\mathrm{T}} \approx 10^{14} \mathrm{~W}$, whereby for this model roughly $99.5 \%$ of the heat are produced in the asthenosphere. The amount of heat which is produced in the asthenosphere was calculated with the help of Eq. (3.27).

For simplicity so far the melt fraction is assumed constant throughout the asthenosphere. Especially if heat transport by melt migration is important a viscosity change with depth similar as in Fig. 1 of Moore (2001) is expected. The lowest viscosity is found at the top of the melting zone, we call this viscosity in the following $\eta_{\mathrm{BL}}$. The tidal dissipation with a depth dependent viscosity due to melt migration is smaller than the tidal dissipation with a constant viscosity $\eta_{\mathrm{c}}$ for $\eta_{\mathrm{c}}=\eta_{\mathrm{BL}}$ and identical asthenosphere thickness as long as $\eta_{\mathrm{BL}}$ is lower than the viscosity at which the tidal dissipation peaks, because then the tidal dissipation decreases if the viscosity increases. We confirmed this relationships by simulations. The convective heat flux is only little affected by a depth dependent viscosity, if the Rayleigh number is defined by the viscosity $\eta_{\mathrm{BL}}$, at least for high Nusselt numbers and a strong viscosity contrast due to the temperature dependence of the viscosity (Dumoulin et al., 1999). Therefore the obtained asthenosphere thicknesses in this section can be viewed as lower bounds, because we need thicker asthenospheres in the case with a depth dependent viscosity due to melt migration to obtain the same tidal dissipation as in the constant viscosity case. Below it will be shown that our determined asthenosphere 


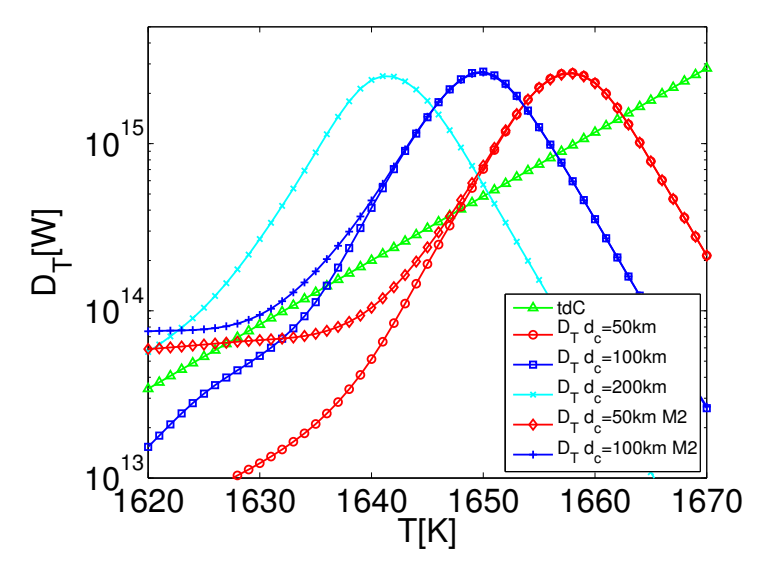

(a)

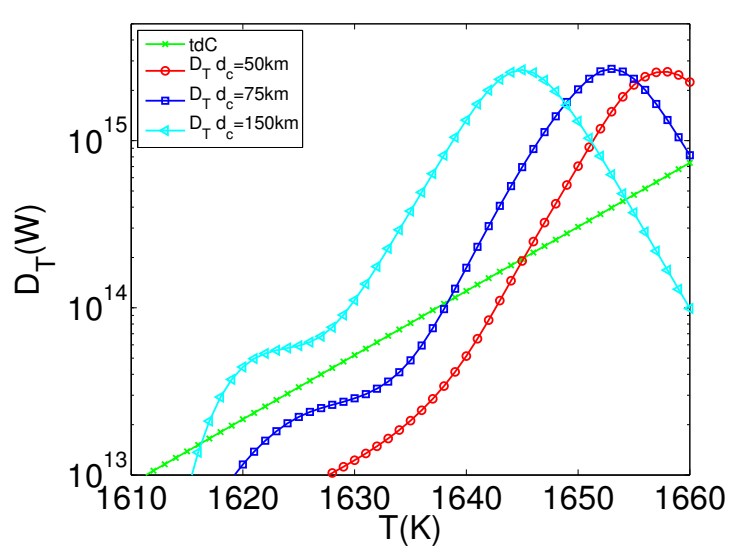

(b)

Figure 3.6.: Tidal dissipation $\left(\mathrm{D}_{\mathrm{T}}\right)$ and transported heat assuming time dependent convection $(\mathrm{tdC})$ for three different asthenosphere thicknesses with a conductivity of $4 \mathrm{Wm}^{-1} \mathrm{~K}^{-1}$ (a) as general assumed in this study and for comparison with a conductivity of $2 \mathrm{Wm}^{-1} \mathrm{~K}^{-1}$ (b). Model parameter see Tables 3.1-3.4, if not otherwise stated in the legend the values for Model 1, M1, were used. $N u(R a)$ according to Eq. (3.29) (tdC). $B=25$.

thicknesses are not only a lower bound, they are furthermore a proper estimate for the thickness with depth dependent viscosity.

Now we want to include melt migration. As already stated a contribution by melt migration is likely. Moore (2001) calculated according to his Eqs. (6) and (7) that all of Io's tidal heat can be transported by melt migration for melt fractions below $20 \%$, depending on the exact parameters. But both crystal size and melt viscosity are uncertain. Carr et al. (1998) assumed a crystal size $\sim 1 \mathrm{~mm}$ and Keszthelyi et al. (2004) suggests that the liquid viscosity $\eta_{1}$ varies between 1 and 400Pas and the density contrast between 520 and $690 \mathrm{~kg} / \mathrm{m}^{3}$ as partial melting varies between $50 \%$ and $20 \%$. Here we calculate with a viscosity $\eta_{1}$ and a density contrast $\Delta \rho$ obtained with the MELTS program (Ghiorso and Sack, 1995), as in Keszthelyi et al. (2004) for the LL-chrondite model (Keszthelyi et al., 2004). In Figs. 3.8(a) and 3.8(b) the heat flux for melt migration was calculated according to Eq. (3.50). With a significant contribution by melt migration to the heat flux the obtained tidal heat depends on the melt fraction coefficient $B$, because in the Darcy law the viscosity of the liquid is used and the tidal dissipation depends on the viscosity of the partially molten rock. If our parameterizations are correct and we have e.g. a $400 \mathrm{~km}$ thick asthenosphere, $B=25$ and grain size $b=5 \mathrm{~mm}$, the obtained tidal dissipation is $9.1 \times 10^{13} \mathrm{~W}$, see Fig. 3.8(a). The contribution by convection can be ignored in this case. These calculations show that taking into account melt migration, stable equilibra are also possible where the slope of the tidal heating curve $D_{T}(T)$ is positive, see e.g. Figs. 3.8(a) and $3.8(\mathrm{~b})$. For a heat transport only by convection this is generally not the case because 


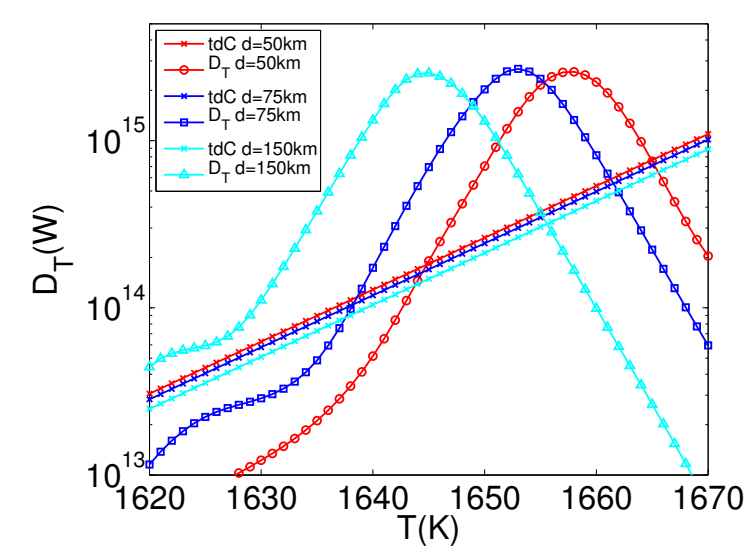

(a)

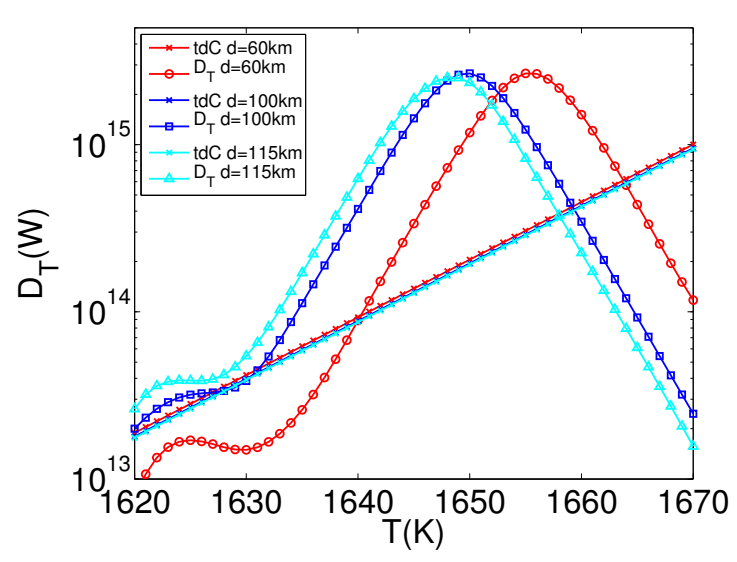

(b)

Figure 3.7.: As in Fig. 3.6(a) but with a $N u(R a)$ scaling according to (3.32) (a) and with the same scaling as in (3.29) but with $\varsigma=0.3$ and $\alpha=1.3$ instead of $1 / 3$ and $4 / 3(\mathrm{~b})$, the asthenosphere thicknesses can be found in the respective legend. $B=25$.

an equilibrium between heat transport and tidal heat flux is stable only if the slope of the tidal heating curve is smaller than the slope of the heat transport curve, otherwise a runaway temperature increase would occur.

From Figs. 3.8(a) and 3.8(b) we can deduce that only for small grain sizes it is possible that convection is the dominant heat transport mechanism, smaller than $2.2 \mathrm{~mm}$ for $B=40$ and even smaller for $B=25$. If the tidal dissipation is $10^{14} \mathrm{~W}$ and convection dominates the heat transport, more than $5 \times 10^{13} \mathrm{~W}$ must be carried by convection. The curves for heat fluxes due to convection and melt migration intersect at $5 \times 10^{13} \mathrm{~W}$ for a grain size of $2.2 \mathrm{~mm}$ in Fig. 3.8(b). The tidal dissipation of $10^{14} \mathrm{~W}$ is realized for an asthenosphere thickness slightly thicker $200 \mathrm{~km}$ for this grain size and the temperature of the intersection point. We can deduce from this figure that a a tidal dissipation of $10^{14} \mathrm{~W}$ for $B=40$ is mainly transported by convection for smaller grain sizes than $2.2 \mathrm{~mm}$ and by melt migration for larger. For $B=25$ the analogue grain size boundary lies at smaller values, see Fig. 3.8(a).

In the remainder of this section we assume that disaggregation happens at a viscosity of $10^{12} \mathrm{Pas}$, which is already a low viscosity for a partial molten mantle with a competent solid matrix (Kohlstedt and Mackwell, 2009). This means we assume $T_{\text {dis }}$ as the temperature corresponding to a viscosity of $10^{12} \mathrm{~Pa}$. Presumably only if a mushy magma ocean, a mix of crystals and melt, could exist smaller viscosities than $10^{12} \mathrm{~Pa}$ s would be possible. If such a mush zone could exist is questionable because of the separation process. Stevenson (2002) predicts that a mushy magma ocean thicker than $20 \mathrm{~km}$ would be unstable over geologic timescales. If smaller viscosities would be attainable equilibria between heat transport and tidal heat production would be possible where the slope of the $D_{T}(T)$ curve 
is negative. But to obtain a tidal dissipation of $10^{14} \mathrm{~W}$ at a higher temperature than the peak temperature the grain size needs to be small, smaller than $0.5 \mathrm{~mm}$ (see Figs. 3.8(a) and $3.8(\mathrm{~b})$ ) and the asthenosphere needs to be large, for $b=0.5 \mathrm{~mm}$ larger than $600 \mathrm{~km}$.

From Fig. 3.9(a) we can deduce that our calculations with a constant melt fraction in the asthenosphere are roughly correct. For this plot we considered melt migration as the sole heat transport mechanism. The largest discrepancy is present for an asthenosphere thickness of $250 \mathrm{~km}$ and $B=25$, the obtained grain size differs in this case by a factor of 2 . The grain size is only poorly known to begin with, therefore this difference is acceptable. Figure 3.9(b) also confirms that our estimated asthenosphere sizes deduced from Fig. 3.6(a) are appropriate for a rough estimate and are a lower bound. Comparing the models with variable and constant viscosity, we find that for a tidal dissipation of $10^{14} \mathrm{~W}$ and any given asthenosphere thickness, the melt fraction is slightly higher at the top of the molten zone for the case of a variable viscosity profile. The difference is strongest for $B=40$ and a $250 \mathrm{~km}$ thick asthenosphere. For this case the melt fraction differs by $10 \%$, which corresponds to a temperature difference of $10 \mathrm{~K}$. The $N u(R a)$ relation is not exactly known, so that a temperature difference of $10 \mathrm{~K}$ is of little importance. Furthermore, because the melt fractions on top are always higher for the variable viscosity profile, our asthenosphere thicknesses are a lower bound.

It can be deduced from Fig. 3.10 that with increasing asthenosphere thickness melt migration becomes more important and the grain size needs to be larger to obtain a tidal dissipation of $10^{14} \mathrm{~W}$. If viscosities smaller than $10^{12} \mathrm{~Pa}$ s would be attainable a dominant contribution by convection is also possible for big asthenosphere thicknesses. Such small viscosities are not considered in Fig. 3.10 but our calculations revealed that it is possible to obtain a tidal dissipation in the range of the observed one for a viscosity smaller than $10^{12} \mathrm{Pas}$, an asthenosphere larger than $785 \mathrm{~km}$ and a grain size smaller than $100 \mu \mathrm{m}$. For asthenosphere thicknesses in the range from $200 \mathrm{~km}$ to $785 \mathrm{~km}$ melt migration is always the dominant mechanism.

\subsubsection{Tidal dissipation of Corot $7 \mathrm{~b}$}

The tidal evolution of Corot $7 \mathrm{~b}$ was computed among others by Rodríguez et al. (2011). It is usually assumed by these authors that $Q^{\prime}=100$ on the grounds that Corot $7 \mathrm{~b}$ is a terrestrial planet (see also e.g. Léger et al. 2011). It will be shown in this section that reasonable assumptions about terrestrial exoplanets lead to values of $Q^{\prime}$ scattered over several orders of magnitude, but $Q^{\prime}=100$ is a realistic approximation. 


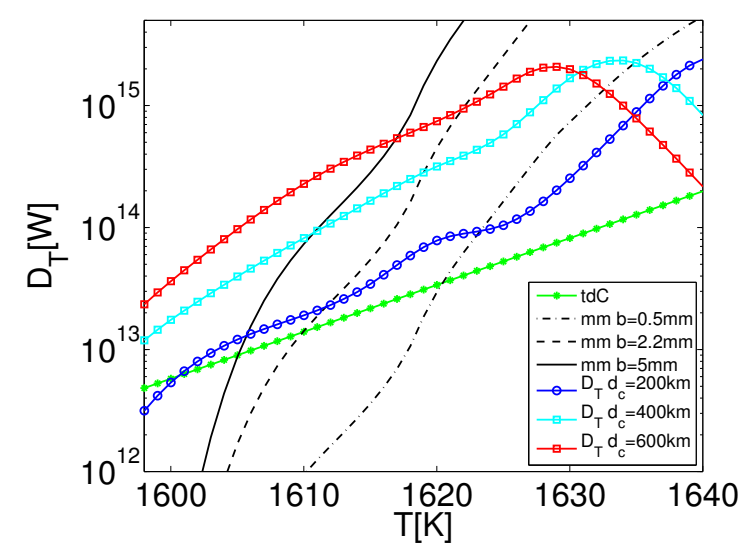

(a)

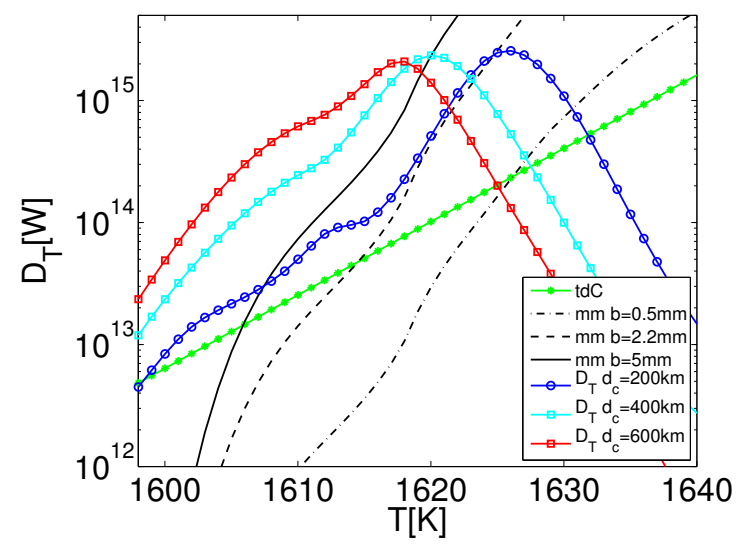

(b)

Figure 3.8.: Heat transported by melt migration $(\mathrm{mm})$ for three different grain sizes, assuming time dependent convection $(\mathrm{tdC})$ and tidal heat $\left(\mathrm{D}_{\mathrm{T}}\right)$ for three different asthenosphere thicknesses and $B=25$ (a) and $B=40(\mathrm{~b})$, model M1. See Tables 3.1-3.4 for model parameters. $N u(R a)$ according to Eq. (3.29) for time dependent convection and melt migration according to Eq. (3.50). Credit: Clausen \& Tilgner, A\&A, 2015, DOI: 10.1051/0004-6361/201526082, reproduced with permission $\odot \mathrm{ESO}$.

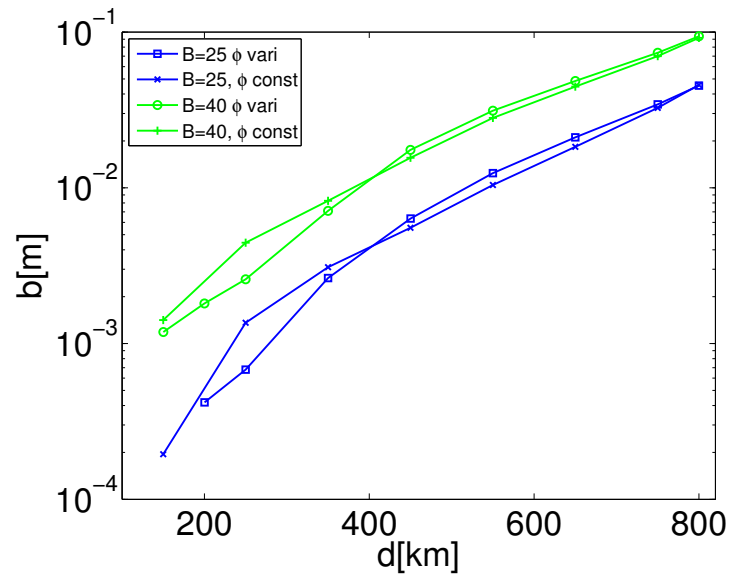

(a)

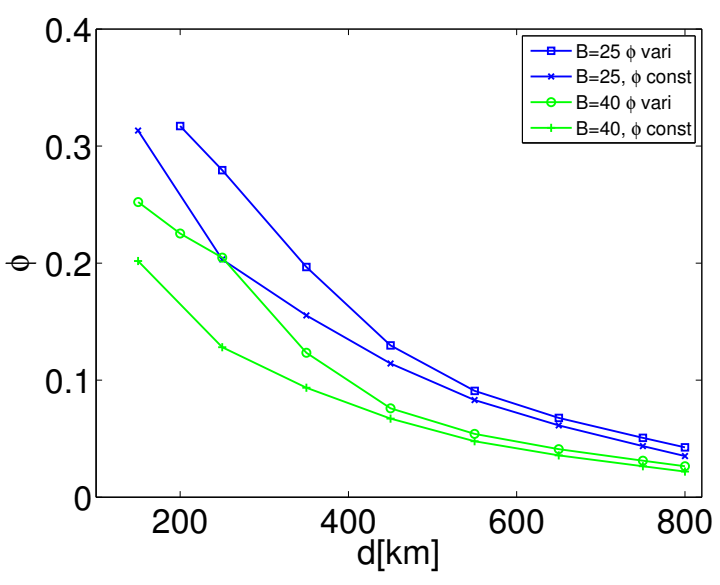

(b)

Figure 3.9.: The grain size $b$ (a) and the corresponding melt fraction at the top of the melting zone (b) for which a tidal dissipation of $10^{14} \mathrm{~W}$ is obtained as a function of asthenosphere thickness, calculated with a constant melt fraction in the asthenosphere ( $\phi$ const) according to Eq. (3.50) and a variable ( $\phi$ vari) as described in Sect. 3.1.2. The convective heat transport is neglected. Credit: Clausen \& Tilgner, A\&A, 2015, DOI: 10.1051/0004-6361/201526082, reproduced with permission $\odot \mathrm{ESO}$. 


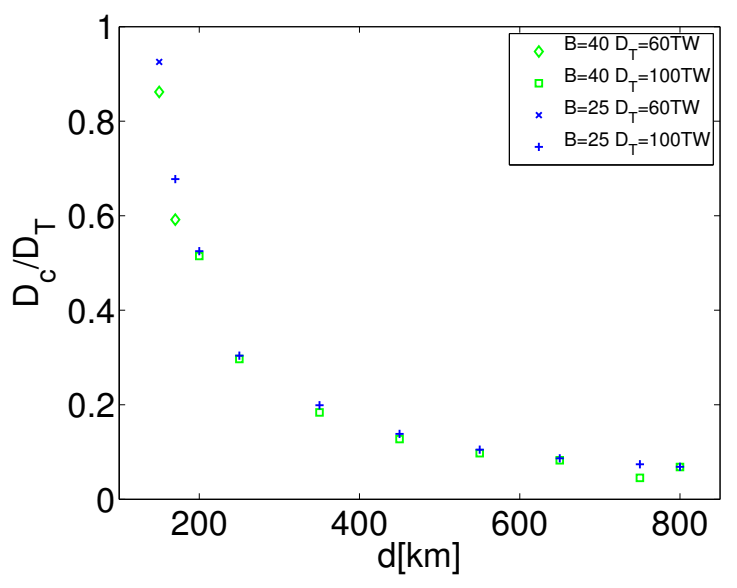

(a)

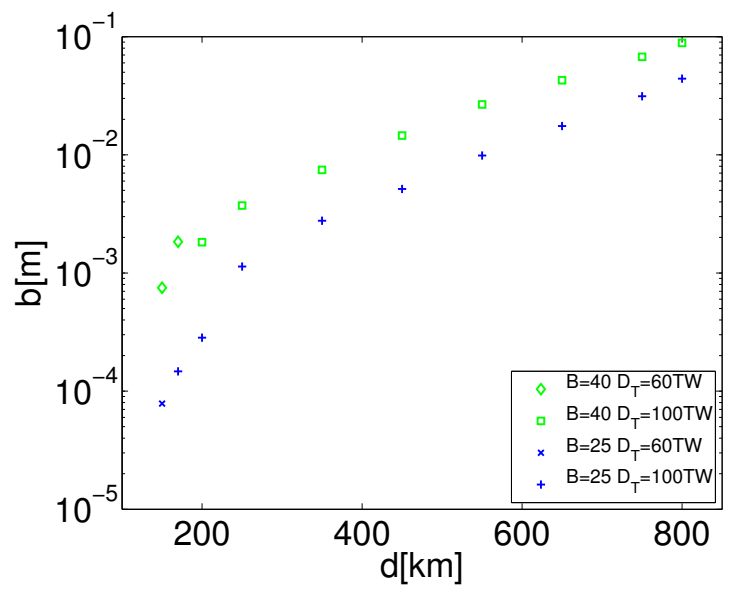

(b)

Figure 3.10.: Amount of heat transported by time dependent convection $\left(D_{c}\right)$ divided by the total heat transported by convection plus melt migration $\left(D_{T}\right)$ as a function of asthenosphere thickness (a). The corresponding grain size (b) was chosen such that the value of tidal dissipation is $10^{14} \mathrm{~W}$, respectively $6 \times 10^{13} \mathrm{~W}$ for small asthenosphere thicknesses, were a value of $10^{14} \mathrm{~W}$ was not accessible. The M1 model was used, model parameter see Tables 3.1-3.4. $N u(R a)$ according to Eq. (3.29) for time dependent convection and melt migration according to Eq. (3.50). Credit: Clausen \& Tilgner, A\&A, 2015, DOI: 10.1051/0004-6361/201526082, reproduced with permission CESO.

In the following, we will obtain $Q^{\prime}$ for objects surrounding Corot 7b's host star, which have either the size and density of Corot $7 \mathrm{~b}$ or the size and density of Io, and which have orbital periods $P$ of either $P=2 \mathrm{~d}$ or $P=6 \mathrm{~d}$.

Various heat transport mechanisms will be considered with the same rheology as for Io in the previous section, model M1, but for the deep mantle we use a lager viscosity, see Table 3.5.

For the case of melt migration, the grain size $b$ equals either $1 \mathrm{~mm}$ or $5 \mathrm{~mm}$. The viscosity $\eta_{1}$ and the density contrast $\Delta \rho$ are again calculated with the MELTS program (Ghiorso and Sack, 1995) as in Keszthelyi et al. (2004) for the LL-chrondite model (see Keszthelyi et al. 2004). The surface temperature is determined with Eq. (3.55). Again $T_{\text {dis }}$ is assumed to be the temperature corresponding to a viscosity value of $10^{12} \mathrm{Pas}$.

The results are summarized in Figs. 3.11 and 3.12, in which $Q^{\prime}$ is given as a function of eccentricity $e$ in a range of eccentricities which may plausibly be encountered during orbital evolution.

For eccentricities too large the tidal forcing is so strong that melt fraction coefficients larger than the melt fraction at the assumed disaggregation point would be necessary to 


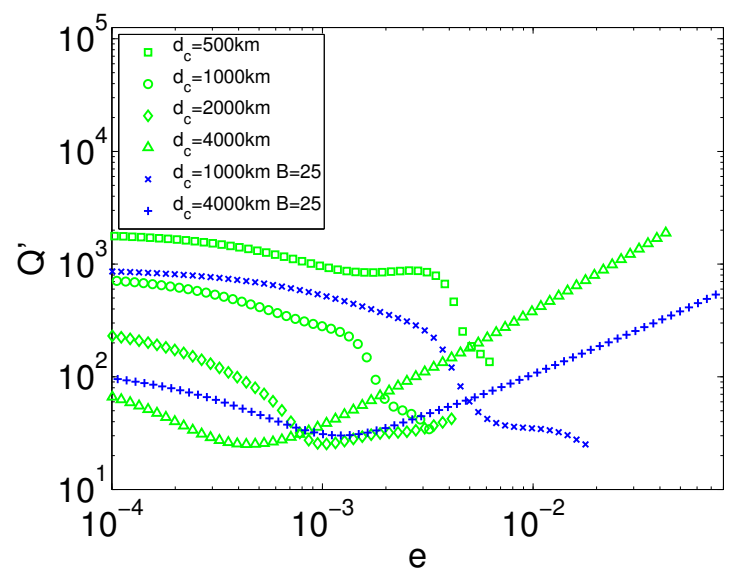

(a)

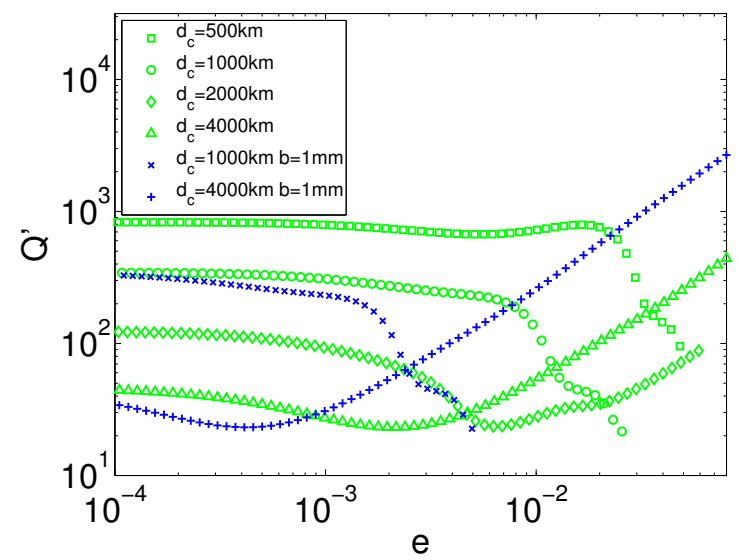

(b)

Figure 3.11.: $Q^{\prime}$ as a function of eccentricity calculated with depth dependent viscosity as described in Sect. 3.1.2, for a planet with the size and density of Corot $7 \mathrm{~b}$ and four different asthenosphere thicknesses. The contribution by a convective heat transport is not included for the calculation of $Q^{\prime}$. Two different orbital periods $P=2 \mathrm{~d}(\mathrm{a})$ and $P=6 \mathrm{~d}(\mathrm{~b})$ were considered. If not otherwise stated $b=5 \mathrm{~mm}$ and $B=40$. Model parameter see Tables 3.1 and 3.3-3.5. Credit: Clausen \& Tilgner, A\&A, 2015, DOI: 10.1051/0004-6361/201526082, reproduced with permission @ESO.

transport the heat. This applies to both heat transport mechanism, convection and melt migration. With increasing eccentricities the tidal dissipation curve is shifted to higher values for temperatures smaller than $T_{\text {dis. }}$. If the eccentricity exceeds a certain value an intersection with the heat transport curve becomes impossible for a given $T_{\text {dis }}$. Therefore no $Q^{\prime}$ values were obtained at large eccentricities. With a heat transport by convection and small eccentricities it is possible that the tidal dissipation is too small at any given temperature to maintain a heat flux compatible with the convective heat transport at that temperature, so that there can be no equilibrium between convective and tidal heat fluxes. For this case no $Q^{\prime}$ can be determined, either.

From Figs. 3.11 and 3.12 it can be deduced that a $Q^{\prime}$ of 100 is a realistic approximation for planets under strong tidal forcing. The values for $Q^{\prime}$ lie mainly between 10 and 1000 . With melt migration as the dominant heat transport mechanism, $Q^{\prime}$ larger than 1000 is only possible for small asthenospheres at small periods or very high eccentricities.

From the figures it can be deduced that there is an influence of heat transport mechanism, eccentricity, orbital period, asthenosphere thickness and size of the object on $Q^{\prime}$. The asthenosphere thickness has a huge influence on $Q^{\prime}$. Because asthenosphere thicknesses are presently not known for any exoplanet it is not useful to describe in detail the dependencies of $Q^{\prime}$ on the other parameters. 


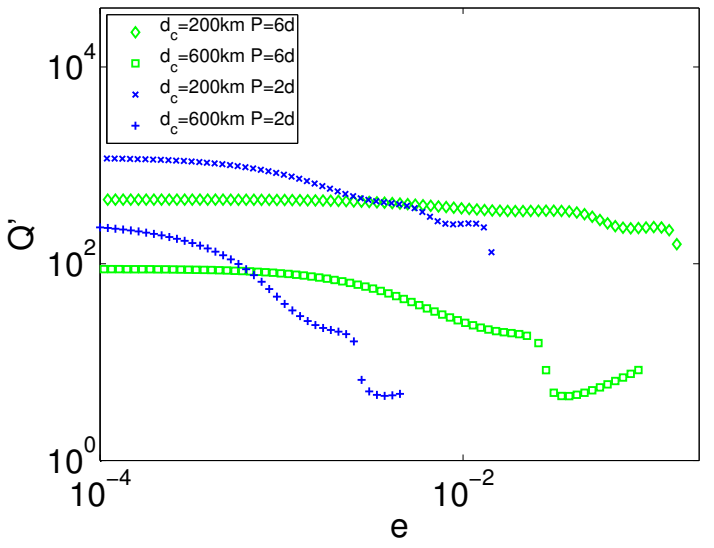

(a)

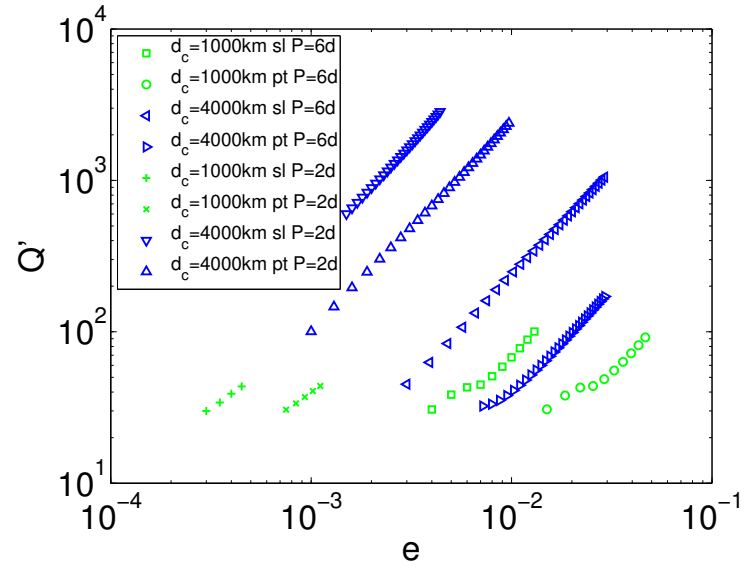

(b)

Figure 3.12.: (a) As in Fig 3.11 but for a planet with the size and density of Io. The respective orbital period is quoted in the legend. See Tables 3.1-3.4 for model parameters. (b) Q' for stagnant lid convection (sl) and plate tectonic convection (pt) as the dominant heat transport mechanism as a function of the eccentricity, for a planet with the size and density of Corot $7 \mathrm{~b}$. Credit: Clausen \& Tilgner, A\&A, 2015, DOI: 10.1051/0004-6361/201526082, reproduced with permission $\odot$ ESO.

\begin{tabular}{ccc}
\hline \hline Symbol & Description & Value \\
\hline$m[\mathrm{~kg}]$ & Mass of the object & $31.06 \times 10^{24 \mathrm{a}}$ \\
$R_{\mathrm{p}}[\mathrm{m}]$ & Radius of the object & $10.07 \times 10^{6 \mathrm{a}}$ \\
$R_{\mathrm{c}}[\mathrm{m}]$ & Core radius & $5.25 \times 10^{6 \mathrm{a}}$ \\
$\rho_{\mathrm{m}}\left[\mathrm{kgm}^{-3}\right]$ & Mantle density & $5490^{\mathrm{a}}$ \\
$L[\mathrm{~W}]$ & Stellar luminosity & $1.84 \times 10^{26 \mathrm{~b}}$ \\
$m_{\mathrm{star}}[\mathrm{kg}]$ & Stellar mass & $1.81 \times 10^{30 \mathrm{~b}}$ \\
$A$ & Planetary albedo & 0.3 \\
$\eta_{\mathrm{dm}}[\mathrm{Pa} \mathrm{s}]$ & Deep mantle viscosity & $10^{25}$ \\
\hline
\end{tabular}

Table 3.5.: Notation and parameters for Corot $7 \mathrm{~b}$. For the planetary albedo we assume a typical value for terrestrial planets. For the deep mantle viscosity we assume a higher value as in Io as expected for a super earth (Tackley et al., 2013).

References. $\quad{ }^{a}$ Wagner et al. (2012); $\quad{ }^{b}$ Léger et al. (2011) 


\subsection{Conclusion and discussion}

In this work the tidal dissipation of rocky planets was investigated for planets with a near equilibrium tide, excluding dynamic tides as the oceanic tides on Earth. Throughout this chapter, "planet" denotes the tidally deformed body, be it a planet or a moon. Tidal forcing is assumed to be strong in the sense that there is a stable equilibrium between tidal dissipation and heat transport due to convection or melt migration. This equilibrium determines the tidal dissipation of the planet.

The method used here is adapted from the method by Moore (2003) (see also Fischer and Spohn 1990). This method is only applicable to planets with a heat budget dominated by tides and solar/stellar radiation. Radiogenic and accretional heat production are ignored. Both the tidal dissipation rate and the convective heat flow depend on the viscosity which in turn depends on temperature. Therefore the temperature must be adjusted such that there is an equilibrium between tidal heat flow and convective heat flow.

The heat flow due to melt migration depends contrary to the convective heat flux and the tidal heat production on the viscosity of the liquid in the partially molten region and not on the viscosity of the partially molten rock.

For the case of melt migration the equilibrium heat production therefore depends on the melt fraction coefficient, and also on the grain size. It is more precise to take into account the depth dependence of the viscosity than using a single viscosity for the partial molten region. For the case of a heat transport dominated by melt migration this is possible by coupling Eq. (3.11) with Eqs. (3.47) and (3.48).

Tidal dissipation was calculated for a body consisting of a solid mantle with a lithosphere, a convecting asthenosphere and a liquid core. Stagnant lid, plate tectonic convection, and melt migration were considered. It was shown that the tidal dissipation of Io can be explained by an equilibrium between convection and tidal heat production. But for the basic assumptions, which entail that the $N u(R a)$ scales according to (3.29) and that the smallest attainable viscosity is $10^{12} \mathrm{Pas}$, a heat transport dominated by convection is only possible for an asthenosphere smaller than $\sim 200 \mathrm{~km}$, but larger than $\sim 100 \mathrm{~km}$, and grain sizes smaller than $2.2 \mathrm{~mm}$, if $B \leq 40$. With an exponent of -1.3 and 0.3 instead of $-4 / 3$ and $1 / 3$ in the $N u(R a)$ relationship Eq. (3.29), as used in Fig. 3.6(a), a heat transport dominated by convection becomes more unlikely, because then the asthenosphere needs to be smaller than $\sim 100 \mathrm{~km}$ for a heat transport dominated by convection. But an asthenosphere smaller than $\sim 100 \mathrm{~km}$ is unlikely, because it requires a presumably too high disaggregation temperature to obtain a tidal dissipation of $10^{14} \mathrm{~W}$. 
Corot $7 \mathrm{~b}$ was also investigated as an example of an exoplanet. The modified tidal quality factor $Q^{\prime}$ of a planet depends on its size. Such a dependence is already mentioned in Efroimsky (2012) as a result of a gravitational effect, whereas here also thermal effects are considered. The radius of exoplanets is usually well constrained by observations, while the eccentricity of their orbit is not. However, $Q^{\prime}$ also depends on eccentricity and orbital frequency as shown above, which also implies that $Q^{\prime}$ varies during orbital history.

Another large uncertainty is introduced by the parameters characterizing the heat transport. These uncertainties add up to a range of plausible values for $Q^{\prime}$ spanning several orders of magnitude. But our results suggest that a $Q^{\prime}$ value of 100 is a realistic approximation for strong tidal forcing. The values for $Q^{\prime}$ lie mainly in the range 10 to 1000 , see Figs. 3.11 and 3.12 . 
4 Inertial modes in shear flow 
The motivation for this chapter is the finding in Chap. 2 that the question if an elliptical instability is possible depends decisively on how turbulent motion has to be considered by the calculation of the decay rate. The growth rate of the elliptical instability depends on the decay rates of the single modes see Eqs. (2.37) and (2.38), or e.g. Kerswell (1994), and thereby on the viscosity. For the viscosity, it is important to know if turbulent convection is present such that a turbulent viscosity has to be introduced or if using only the molecular viscosity is appropriate. In Sect. 2.3.1 it is written how to model turbulent viscosity. The intention for this section was to determine more precisely how inertial modes are affected by turbulent motion. It will now be explained what the original plan was, which we were not able to complete and is left for future studies. The reference frame is rotating with $\Omega_{s} \hat{\mathbf{z}}$. We consider a region confined in the z-direction. The basic flow has only a velocity component in the $\mathrm{x}$-direction and depends only on the $\mathrm{z}$ component, $u_{0}(z) \hat{\mathbf{x}} . \quad u_{0}(z)$ is randomly chosen, but nondimensionalized such that $u_{0}(z) \in[-1,1]$ and the arithmetic mean value of the basic flow has to be zero. For the perturbation velocity, we make an ansatz for inertial modes. The problem reduces then to a one dimensional problem, which can be solved with a shooting method. From the imaginary part of the frequency of the inertial mode, which we determine directly by the shooting method, we can calculate the turbulent viscosity. In the following sections the Euler equation is considered. For a validation of the shooting method, we perform an analytical calculation of a simplified version of the problem described above with $u_{0}(z)=-U$ for $z<0$ and $u_{0}(z)=U$ for $z \geq 0$. We numerically treat the problem with $u_{0}(z)=U \tanh \left(F_{\mathrm{s}} z\right)$ to avoid the velocity jump. We expected that the resulting profiles resemble each other for suitable chosen $F_{\mathrm{s}}$ and identical remaining parameters.

\subsection{Equations for fluid motion in Ekman-Couette flow}

We consider an incompressible fluid. The governing equations of fluid motion are the Navier-Stokes equations

$$
\begin{array}{r}
\partial_{t} \mathbf{u}+\mathbf{u} \cdot \nabla \mathbf{u}+2 \Omega_{0} \hat{\mathbf{z}} \times \mathbf{u}=-\frac{1}{\rho} \nabla p+\nu \Delta \mathbf{u}, \\
\nabla \cdot \mathbf{u}=0,
\end{array}
$$

where $\mathbf{u}(x, y, z, t)$ is the flow velocity field and $p(x, y, z, t)$ is the pressure field. The following proceeding with the governing equations is similar to the formulation in Shi et al. (2014). We nondimensionalize the equations by taking the half gap distance between the 
two plates $D / 2$ as the length unit and $D / 2 U$ as the time unit,

$$
l=l^{*} D / 2, \quad t=t^{*} D / 2 U, \quad \mathbf{u}=\mathbf{u}^{*} U, \quad p=p^{*} \rho U^{2} .
$$

Henceforth, we will omit the stars at the symbols, which indicate the nondimensionality in the equations. The nondimensional form of Eqs. (4.1) can now be written as

$$
\begin{array}{r}
\partial_{t} \mathbf{u}+\mathbf{u} \cdot \nabla \mathbf{u}+\frac{1}{R o} \hat{\mathbf{z}} \times \mathbf{u}=-\nabla p+\frac{1}{R e} \Delta \mathbf{u}, \\
\nabla \cdot \mathbf{u}=0,
\end{array}
$$

with the Reynolds number and the Rossby number

$$
R e=\frac{U D}{2 \nu}, \quad R o=\frac{U}{\Omega_{0} D},
$$

respectively. The base velocity profile has the form of $\left[u_{0}(z), 0,0\right]$. The velocity can be decomposed into its value in the basic state and a perturbation, which is considered to be small: $\mathbf{u}=u_{0}(z) \hat{\mathbf{x}}+\mathbf{u}^{\prime}$. By taking the curl of Eq. (4.4) once and twice, respectively, and then projecting into the $\mathrm{z}$ direction, we obtain the linearized equations for the perturbation values $(w, \eta)$,

$$
\begin{aligned}
\partial_{t} \nabla^{2} w+u_{0} \partial_{x} \nabla^{2} w-\left(\partial_{x} w\right)\left(\partial_{z}^{2} u_{0}\right) & =\frac{1}{R e} \nabla^{4} w-\frac{1}{R o} \partial_{z} \eta \\
\partial_{t} \eta+u_{0} \partial_{x} \eta-\left(\partial_{y} w\right)\left(\partial_{z} u_{0}\right) & =\frac{1}{R e} \nabla^{2} \eta+\frac{1}{R o} \partial_{z} w
\end{aligned}
$$

with $w$ the z-component of the velocity $\mathbf{u}^{\prime}$ and $\eta$ the z-component of the perturbation vorticity.

Henceforth, we consider the inviscid case. We want to treat the system analytically and numerically. For the analytical case we assume for simplification instead of a continuous varying basic velocity field, a velocity field that in the upper region $(z>0)$ has constant velocity $U$ and in the lower region $(z<0)-U$. We have a discontinuity in this case and need jump conditions to solve the problem. The derivation of the governing analytical equations can be found in the results, Sect. 4.2.

We numerically solve the problem of a continuous varying velocity field which has the form $u_{0}(z)=U \cdot \tanh \left(F_{\mathrm{s}} z\right)$. We expect that the numerical calculations approximates the analytical calculation for suitable $F_{\mathrm{s}}$ and otherwise the same parameter. So we can use 
the analytical calculation to check our numerical code. We start from the equations

$$
\begin{array}{r}
\partial_{t} \nabla^{2} w+u_{0} \partial_{x} \nabla^{2} w-\left(\partial_{x} w\right)\left(\partial_{z}^{2} u_{0}\right)=-\frac{1}{R o} \partial_{z} \eta \\
\partial_{t} \eta+u_{0} \partial_{x} \eta-\left(\partial_{y} w\right)\left(\partial_{z} u_{0}\right)=\frac{1}{R o} \partial_{z} w
\end{array}
$$

which is the inviscid counterpart of Eq. (4.7). Substituting the ansatz

$$
w=\hat{w}(z) e^{\mathrm{i}\left(\omega t-k_{x} x-k_{y} y\right)}, \quad \eta=\hat{\eta}(z) e^{\mathrm{i}\left(\omega t-k_{x} x-k_{y} y\right)},
$$

into Eq. (4.9) and isolating $\hat{\eta}$ gives

$$
\hat{\eta}=\frac{\left(\partial_{z} u_{0}\right) k_{y} \hat{w}-\mathrm{i}\left(\partial_{z} \hat{w} / R o\right)}{\omega-u_{0}(z) k_{x}} .
$$

Substituing this and the ansatz (4.10) into Eq. (4.8) gives

$$
\omega\left(\hat{\nabla}^{2} \hat{w}\right)-u_{0} k_{x} \hat{\nabla}^{2} \hat{w}+k_{x} \hat{w}\left(\partial_{z}^{2} u_{0}\right)-\frac{1}{R o} \partial_{z}\left(\frac{\mathrm{i} k_{y} \hat{w}\left(\partial_{z} u_{0}\right)+\left(\partial_{z} \hat{w} / R o\right)}{\omega-u_{0}(z) k_{x}}\right)=0
$$

with $\hat{\nabla}^{2}=\partial_{z}^{2}-\left(k_{x}^{2}+k_{y}^{2}\right)$. Now we have eliminated $\hat{\eta}$ in the equation. This last equation can be solved with a shooting method (Press et al., 1992) to obtain $\hat{w}(z)$ and the eigenvalue $\omega$, for certain $k_{x}, k_{y}, R o$ and $F_{\mathrm{s}}$.

\subsection{Results}

For the inviscid, analytical case Eq. (4.7) can be written as

$$
\begin{array}{r}
\partial_{t} \nabla^{2} u \pm U \partial_{x} \nabla^{2} w=-\frac{1}{R o} \partial_{z} \eta \\
\partial_{t} \eta \pm U \partial_{x} \eta=\frac{1}{R o} \partial_{z} w
\end{array}
$$

with the upper sign for $z>0$ and the lower for $z<0$. These two equations can be combined to eliminate $\eta$

$$
\left(\partial_{t} \pm U \partial_{x}\right)\left(\partial_{t} \pm U \partial_{x}\right) \nabla^{2} w=-\frac{1}{R o} \partial_{z}\left(\partial_{t} \pm U \partial_{x}\right) \eta=-\frac{1}{R o^{2}} \partial_{z} w
$$

As we are interested in inertial modes, we make the ansatz for the perturbation velocity

$$
w=\hat{w}(z) e^{\mathrm{i}\left(\omega t-k_{x} x-k_{y} y\right)},
$$


where $k_{x}$ and $k_{y}$ are the wavenumbers in the $\mathrm{x}$ and $\mathrm{y}$ direction and

$$
\hat{w}(z)=A_{ \pm} e^{\mathrm{i} \gamma_{ \pm} z}+B_{ \pm} e^{-\mathrm{i} \gamma_{ \pm} z}
$$

Substituting this ansatz in Eq. (4.15) gives

$$
\gamma_{ \pm}=k\left(\frac{1}{\left(\omega \mp k_{x} U\right)^{2} R o^{2}}-1\right)^{-1 / 2}
$$

with $k=\sqrt{k_{x}^{2}+k_{y}^{2}}$. Because we consider an inviscid flow, the velocity has only to satisfy the no penetration condition at the liquid-solid boundary $w(z= \pm d / 2)=0$ and therefore it needs to be $\hat{w}(z= \pm d / 2)=0$ :

$$
A_{ \pm} e^{ \pm \mathrm{i} \gamma_{ \pm}}+B_{ \pm} e^{\mp \mathrm{i} \gamma_{ \pm}}=0
$$

Furthermore, we need jump conditions at the velocity discontinuity. We expect that here the continuity of particle displacement needs to be satisfied. This condition differs from the condition of a continuous particle velocity because of the convective term. The correctness of this condition has been pointed out before by Miles (1957), Ribner (1957) and Ingard (1959) for acoustic wave reflection and transmission at an interface between two moving fluids, but without rotation. With $S(x, y, t)$ as the z-coordinate of the free boundary (the discontiuity), a particle on the free boundary has the z-coordinate $S$, it is

$$
w=\frac{D}{D_{t}} S=\partial_{t} S+u \partial_{x} S+v \partial_{y} S
$$

with $u$ and $v$ the velocities in $\mathrm{x}$ - and $\mathrm{y}$-direction, respectively. We consider small perturbations, therefore we could linearize the above equation, this gives

$$
w=\left(\partial_{t} \pm U \partial_{x}\right) S
$$

and for the limit to \pm 0

$$
\begin{aligned}
& \left.w\right|_{0+}=\left(\partial_{t}+U \partial_{x}\right) S, \\
& \left.w\right|_{0-}=\left(\partial_{t}-U \partial_{x}\right) S .
\end{aligned}
$$

Therefore, it is

$$
\left.\left(\partial_{t}-U \partial_{x}\right) w\right|_{0^{+}}=\left.\left(\partial_{t}+U \partial_{x}\right) w\right|_{0^{-}}=\left(\partial_{t}+U \partial_{x}\right)\left(\partial_{t}-U \partial_{x}\right) S
$$

with Eq. (4.16) this gives the condition

$$
\left(\omega+U k_{x}\right)\left(A_{+}+B_{+}\right)=\left(\omega-U k_{x}\right)\left(A_{-}+B_{-}\right) .
$$


Now we want to derive a fourth condition which can be deduced from the fact that the pressure needs to be continuous at the free boundary. For the pressure, we make the ansatz

$$
p=\hat{p}(z) e^{\mathrm{i}\left(\omega t-k_{x} x-k_{y} y\right)},
$$

with $\hat{p}(z)=\left(C_{ \pm} e^{\mathrm{i} \gamma_{ \pm} z}+D_{ \pm} e^{-\mathrm{i} \gamma_{ \pm} z}\right)$. The linearized Euler equations read

$$
\begin{array}{r}
\partial_{t} \mathbf{u}+\frac{1}{R o} \hat{\mathbf{z}} \times \mathbf{u} \pm U \partial_{x} \mathbf{u}=-\nabla p, \\
\nabla \cdot \mathbf{u}=0,
\end{array}
$$

and the z-component of the momentum equation, Eq. (4.26),

$$
\partial_{t} w \pm U \partial_{x} w=-\partial_{z} p
$$

Calculating the divergence of the Euler equation gives

$$
-\nabla^{2} p=-\frac{\eta}{R o}
$$

this gives together with Eq. (4.13)

$$
\left(\partial_{t} \pm U \partial_{x}\right) \nabla^{2} p=\frac{\partial_{z} w}{R o^{2}}
$$

Substituing Eq. (4.25) in Eq. (4.30) gives

$$
\begin{gathered}
C_{ \pm}=\frac{\omega \mp k_{x} U}{\gamma_{ \pm}}(-A \pm), \\
D_{ \pm}=\frac{\omega \mp k_{x} U}{\gamma_{ \pm}} B_{ \pm} .
\end{gathered}
$$

Now with the continuity of $p$ at the velocity discontinuity, we get the fourth condition

$$
\frac{\omega-k_{x} U}{\gamma_{+}}\left(-A_{+}+B_{+}\right)=\frac{\omega+k_{x} U}{\gamma_{-}}\left(-A_{-}+B_{-}\right) .
$$

Combining the four deduced conditions gives

$$
-\left(\gamma_{+} \cdot \frac{d}{2}\right) \tan \left(\gamma_{+} \cdot \frac{d}{2}\right)\left(\omega+U k_{x}\right)^{2}=\left(\gamma_{-} \cdot \frac{d}{2}\right) \tan \left(\gamma_{-} \cdot \frac{d}{2}\right)\left(\omega-U k_{x}\right)^{2}
$$

This equation together with Eq. (4.18) will be used to calculate $\omega$ and $\gamma_{ \pm}$at certain $k_{x}$, $k_{y}, d, R o$ and $U$.

We now want to check our analytical solution. This can be performed by calculating analytically $\omega$ and $\gamma_{ \pm}$for given $k_{x}, k_{y}, d, R o$ and $U$ as described above. Then we solve 


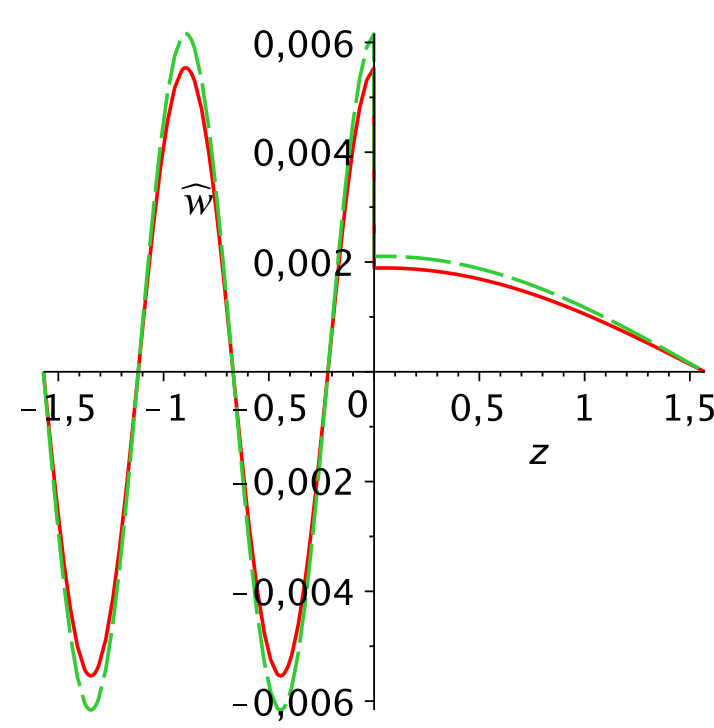

(a)

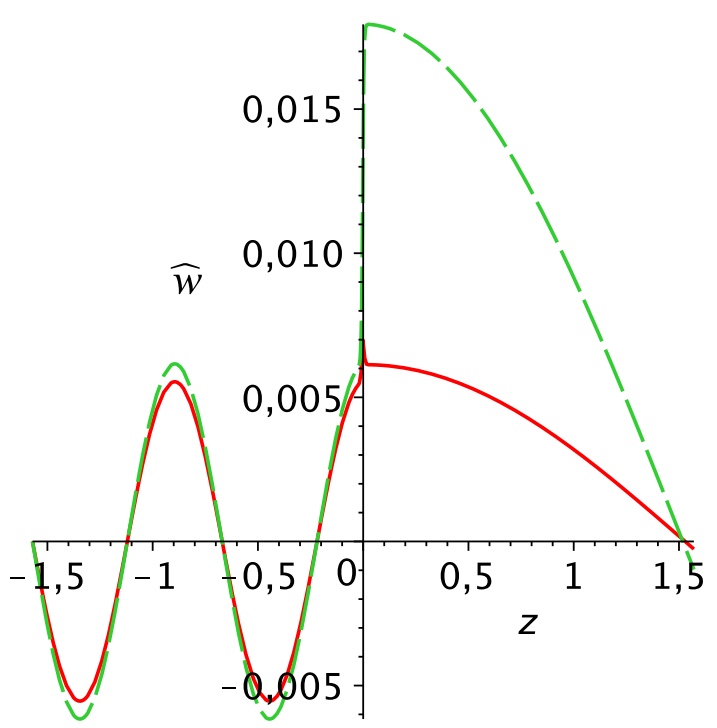

(b)

Figure 4.1.: Real (red solid line) and imaginary part (green dashed line) of the velocity component $\hat{w}$ as a function of height for the analytical calculation (left) and the numerical calculation according to Eq. (4.12) (right). Wave parameters are $k_{x}=3, k_{y}=1$ and $\omega \approx 0.611$ and it is $U=0.1$ and for the numerical calculation $F_{s}=160$.

numerically with MAPLE the Eq. (4.12) for the analytical calculated $\omega$, the other parameters are the same, but $u_{0}(z)=U \tanh \left(F_{\mathrm{s}} z\right)$ because with a discontinuous shear flow a numerical calculation is not possible. The result of an example calculation can be seen in Fig. 4.1. From this figure it can be deduced that in the lower part of the shear flow, where we started our numerical integration, the analytical and numerical solution show a good match. In the upper part $(z>0)$, the two solutions do not agree. From this we can deduce that presumably the jump condition is not correct, but to verify our calculation we also conduct an other derivation of an equation with the velocity component $w$ as the only variable and solve this numerically, instead of Eq. (4.12). To obtain this equation, we combine Eq. (4.4) which corresponds to 3 scalar equation and Eq. (4.5) such that we get an equation for the velocity component $w$ alone. We use MAPLE to perform the necessary operations on the equations. The resulting equation is a bit lengthy, therefore we display the commands and resulting equations in Fig. F.1. In Figs. 4.2 and 4.3(b), $w$ and $p$ according to Eqs. eqv and pw (see Fig. F.1(b)), respectively, is plotted. We see Fig. 4.2 matches well with Fig. 4.1(b). In Fig. 4.3(a) $c_{3}$ is plotted, which is given by

$$
c_{3}=\left(\omega+u_{0}(z) k_{x}\right) \hat{w}(z)
$$

If the condition according to Eq. (4.24) would be satisfied by the numerical calculation, $c_{3}$ needs to be continuous. From Fig. 4.3(a) we can see that this is not the case, but 
the pressure is continuous in the numerical calculation, see Fig. 4.3(b). From this we can deduce that the condition of pressure continuity at the free boundary is correct and the error lies presumably in the condition of particle displacement.

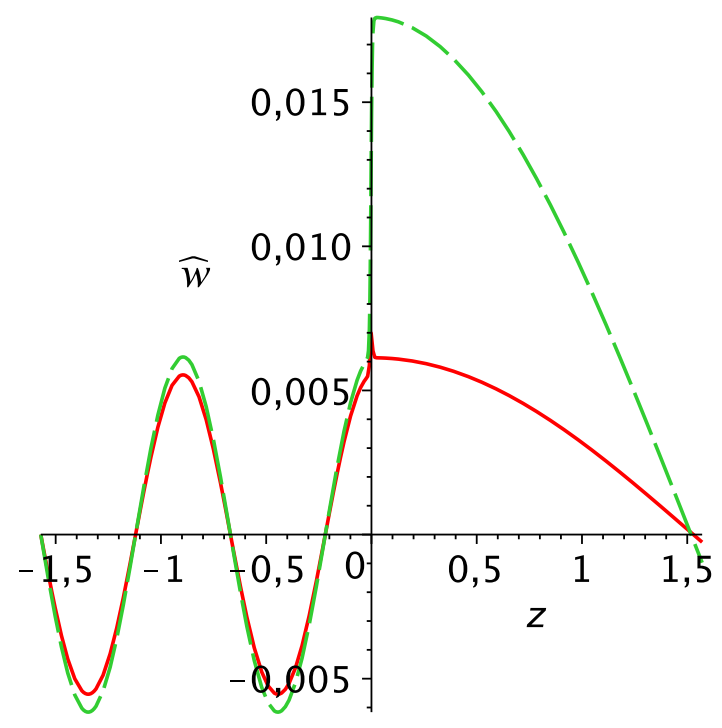

Figure 4.2.: Real (red solid line) and imaginary part (green dashed line) of the velocity component $\hat{w}$ as a function of height for the numerical calculation according to the Eq. eqv, displayed in Fig. F.1. Parameters as in Fig 4.1.

\subsection{Conclusion and discussion}

In this chapter we considered a basic velocity profile $u_{0}(z) \hat{\mathbf{x}}$ in the form of a stairstep, $u_{0}(z)=-U$ for $z<0$ and $u_{0}(z)=U$ for $z \geq 0$. The system is rotating with $\Omega_{s} \hat{\mathbf{z}}$, therefore the propagation of inertial modes is possible. The flow is confined in the z-direction. The governing inviscid equations were solved analytically with an ansatz for the perturbation velocity describing inertial modes. At the velocity discontinuity jump conditions have to be satisfied. A similar problem was solved numerically with MAPLE, where the discontinuous velocity profile was substituted by a continuous profile $u_{0}(z)=U \tanh \left(F_{\mathrm{s}} z\right)$. We expected that for appropriate $F_{\mathrm{S}}$ and otherwise the same parameters as in the analytical case the numerically calculated velocity profiles would resemble the analytically calculated velocity profile. But this was not the case, see Fig. 4.1. Because in the lower part $z<0$, where we started our numerical calculations, the profiles are similar, and in the upper part $z>0$ the solutions do not agree, we assume that the error lies in the jump conditions for the analytical case. We can see in Fig. 4.3 that the condition for the continuity of particle displacement is not fulfilled numerically contrary to the condition of constant pressure, 


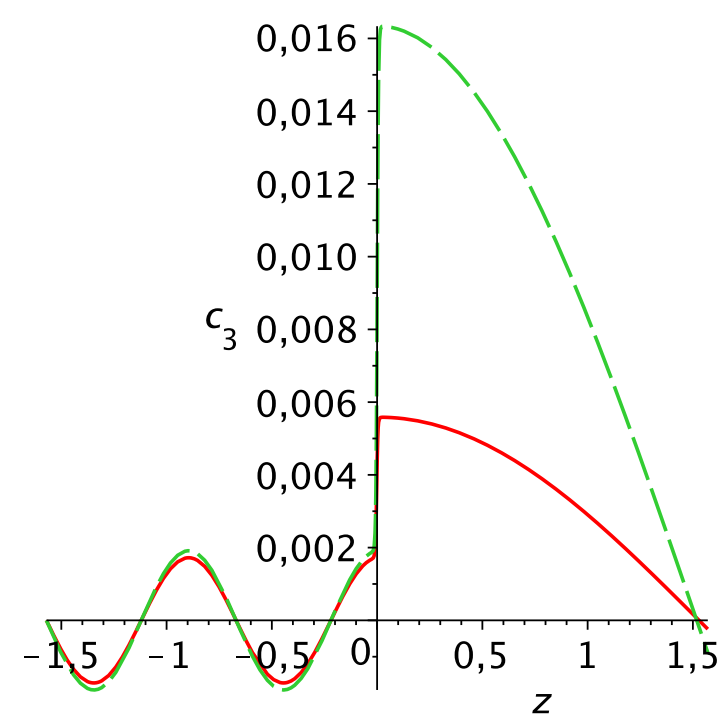

(a)

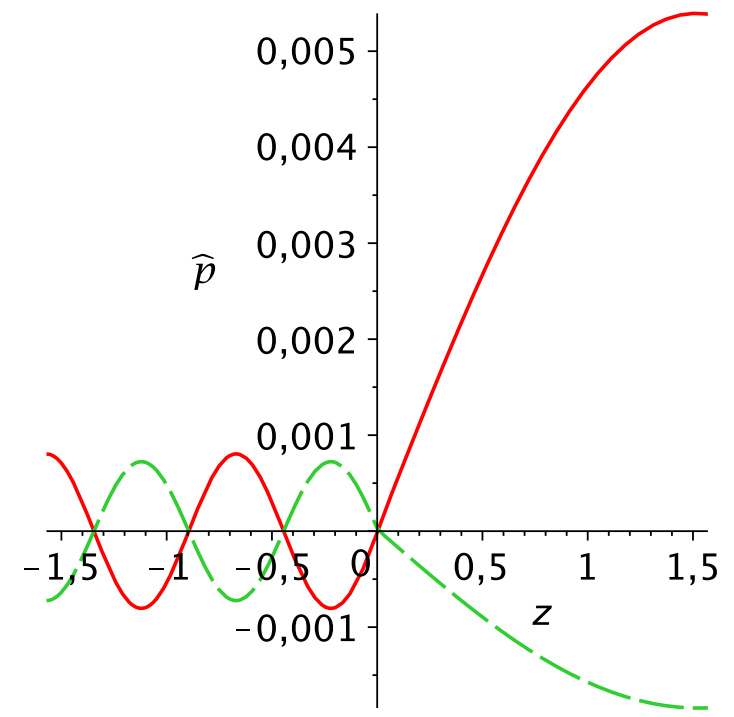

(b)

Figure 4.3.: Real (red solid line) and imaginary part (green dashed line) of $c_{3}$, Eq. (4.34), as a function of height for the numerical calculation according to the Eq. eqv, displayed in Fig. F.1, (left) and the real and imaginary part of the corresponding pressure $\hat{p}$ according to Eq. pw, displayed in Fig. F.1, (right). Parameters as in Fig 4.1.

which indicates that this condition in incorrect. The condition of continuity of particle displacement in the used form may be correct without rotation, but it needs to be checked if the rotation changes this condition. 
5 Summary 
This thesis is divided into three main parts, in accordance with the last three chapters. The common feature of these three parts is that they all aim to better understand the tidal dissipation in planets and/or stars.

In Chap. 2 we investigated the elliptical instability under the influence of compressibility. To determine the growth rates we studied the linear stability of the flow. We solved the Euler equations. The viscosity was then introduced in our calculations heuristically. We assumed a power law for the radial dependence of the background density. Together with the use of the anelastic approximation, this enabled us to apply semi-analytical methods for solving the equations. We found that the growth rate of a certain mode combination depends on the compressibility. However, the influence of the compressibility is negligible for the growth rate maximized over all possible modes if viscous bulk damping effects can be neglected. The growth rate maximized over all possible modes determines the stability of the flow. From this we can deduce that the stability limits for the compressible flow are the same as for the incompressible flow as long as the effects of viscous bulk dissipation are negligible. But depending on the ratio $\Omega_{P} / \Omega_{F}$, with $\Omega_{F}$ the spin rate of the central object in the frame of the rotating tidal perturber and $\Omega_{P}$ the orbiting frequency of the tidal perturber in the inertial frame, certain pairs of modes resonate with each other. Because the size of the bulk damping term depends on the modes that resonate with each other, the growth rate of the viscous flow depends on the compressibility. However the difference in the stability limit caused by the compressibility is in general negligible against the uncertainty in the estimation of the turbulent viscosity. Furthermore, as the correct choice of the boundary conditions for stars and giant planets is unclear, another uncertainty is introduced.

In Chap. 3 we investigated the tidal dissipation of rocky planets for strong tidal forcing. Tidal dissipation depends on the heat transport mechanism. For strong tidal forcing, one can obtain an equilibrium between heat transport by convection and heat production by tidal dissipation, which determines the tidal dissipation. By this means we checked whether convection is the dominant heat transport mechanism in Io. The tidal dissipation also depends on the interior model of Io. We considered various asthenosphere thicknesses and determined which of these gives results that are compatible with observations. Furthermore, we determined the modified tidal quality factors $\left(Q^{\prime}\right)$ for Corot- $7 \mathrm{~b}$ for various orbital parameters but such that tidal forcing is strong and used convection and melt migration as a possible heat transport mechanism. We did the same for a hypothetical planet with the size and density of Io on the orbit of Corot-7b. We found that a heat transport dominated by convection in Io is possible, but for this the grains size needs to be smaller than $2.2 \mathrm{~mm}$. For larger grain sizes melt migration is the dominant heat transport mechanism. Additionally, we found that Io's asthenosphere must be thicker than $100 \mathrm{~km}$. 
The computation of the modified tidal quality factors $\left(Q^{\prime}\right)$ for Corot-7b and a planet with the size and density of Io on the orbit of Corot-7b show that $Q^{\prime}$ is scattered over several orders of magnitude, but a value of 100 for $Q^{\prime}$ is an acceptable estimate for a rocky planet under strong tidal forcing. For Corot-7b and the planet with the size and density of Io we also used a model with different asthenosphere thicknesses. Our calculations revealed that the asthenosphere thickness has a huge influence on $Q^{\prime}$. Asthenosphere thicknesses for exoplanets are presently unknown, therefore a description about the dependencies of $Q^{\prime}$ on the size of the planet, its orbital eccentricity, and heat transport mechanism was not stated.

In Chap. 4 we investigated inertial modes in shear flow. The aim was to better estimate the influence of turbulence on inertial modes in order to better quantify the turbulent viscosity, inspired by the findings in Chap. 2. This task remains unfinished and is left for future studies. In this part we considered a basic velocity profile $u_{0}(z) \hat{\mathbf{x}}$ in the form of a stairstep, $u_{0}(z)=-U$ for $z<0$ and $u_{0}(z)=U$ for $z \geq 0$ and one with a continuous profile $u_{0}(z)=U \tanh \left(F_{\mathrm{s}} z\right)$. The system rotates with $\Omega_{s} \hat{\mathbf{z}}$ and the flow is confined in the zdirection. The governing inviscid equations were solved analytically with an ansatz for the perturbation velocity describing inertial modes for the stairstep profile and numerically, for the continuous profile. We expected that for an appropriate $F_{\mathrm{s}}$ and otherwise the same parameters as in the analytical case, the numerically calculated velocity profiles would resemble the analytically calculated velocity profile. But this was not the case. For the analytical case, two jump conditions must be fulfilled: continuity of particle displacement and continuity of pressure. Numerically, the latter condition is fulfilled but not the former. We therefore assume that the condition of continuity of particle displacement cannot be correct with rotation; without rotation it is used often and its validity has been widely recognized for a long time (Miles, 1957; Ribner, 1957; Ingard, 1959). 


\section{A Eigenvalue problem for the power- law density sphere}

This derivation is in accordance with the work in $\mathrm{Wu}$ (2005a) (details can be found therein). The following definition will be used

$$
\zeta=-\frac{\omega+k}{2(1+\Omega)}
$$

Equation (2.25) can now be written as

$$
\boldsymbol{\xi}+\frac{\mathrm{i}}{\zeta} \hat{\mathbf{z}} \times \boldsymbol{\xi}=\nabla \tilde{\psi}
$$

with $\tilde{\psi}=\psi /(\omega+k)^{2}$. The subscript $j$, which indicates the respective mode, is omitted here. The relationship between $\boldsymbol{\xi}$ and $\tilde{\psi}$

$$
\boldsymbol{\xi}=\frac{1}{1-\frac{1}{\zeta^{2}}}\left(1-\frac{\mathrm{i}}{\zeta} \hat{\mathbf{z}} \times-\frac{1}{\zeta^{2}} \hat{\mathbf{z}} \hat{\mathbf{z}} \cdot\right) \nabla \tilde{\psi}
$$

can be deduced by operating on Eq. (A.2) with $\hat{\mathbf{z}} \cdot$ and $\hat{\mathbf{z}} \times$. Combining this equation with Eq. (2.27) gives

$$
\nabla^{2} \tilde{\psi}-\frac{1}{\zeta^{2}} \frac{\partial^{2} \tilde{\psi}}{\partial z^{2}}=\frac{1}{H}\left(\frac{\partial \tilde{\psi}}{\partial r}-\frac{1}{\zeta^{2}} \cos \theta \frac{\partial \tilde{\psi}}{\partial z}-\frac{k \frac{1}{\zeta}}{r} \tilde{\psi}\right)
$$

with the zenith angle $\bar{\theta}$ and $\cos \bar{\theta}=\bar{z} / \bar{r}$, with $\bar{z}$ being the height along the rotational axis. The partial derivatives here are to be understood as $\partial / \partial \bar{r}=\partial /\left.\partial \bar{r}\right|_{\bar{\theta}}, \partial / \partial \bar{\theta}=\partial /\left.\partial \bar{\theta}\right|_{\bar{r}}$, $\partial / \partial \bar{z}=\partial /\left.\partial \bar{z}\right|_{\bar{s}}$, and $\partial / \partial \bar{s}=\partial /\left.\partial \bar{s}\right|_{\bar{z}}$, with $\bar{s}$ the cylindrical radius. The overline denotes that the coordinates are chosen with respect to a reference frame rotating with $\Omega_{\mathrm{F}}+\Omega_{\mathrm{p}}$. In this appendix the coordinates are always chosen in that way, therefore we omit the overline henceforth. 
Now following Bryan a set of ellipsoidal coordinates $\left(x_{1}, x_{2}, \varphi\right)$ will be used, $x_{1}$ and $x_{2}$ depend on the value of $\zeta$. The left hand side of Eq. (A.4) is separable in these coordinates. The right side of the same equation is generally inseparable, but for the two special cases of uniform and power law density profile the right hand side becomes separable. The ellipsoidal coordinates are related to the Cartesian coordinates as

$$
x=\left[\frac{\left(1-x_{1}^{2}\right)\left(1-x_{2}^{2}\right)}{1-\zeta^{2}}\right]^{\frac{1}{2}} \cos \varphi, \quad y=\left[\frac{\left(1-x_{1}^{2}\right)\left(1-x_{2}^{2}\right)}{1-\zeta^{2}}\right]^{\frac{1}{2}} \sin \varphi, \quad z=\frac{x_{1} x_{2}}{\zeta} .
$$

with $x_{1} \in[\zeta, 1], x_{2} \in[-\zeta, \zeta]$, and $\varphi$ is the azimuthal angle with $\varphi \in[0,2 \pi]$. The cylindrical and spherical radii are given by

$$
\begin{aligned}
& s^{2}=x^{2}+y^{2}=\frac{\left(1-x_{1}^{2}\right)\left(1-x_{2}^{2}\right)}{1-\zeta^{2}}, \\
& r^{2}=x^{2}+y^{2}+z^{2}=1-\frac{\left(x_{1}^{2}-\zeta^{2}\right)\left(\zeta^{2}-x_{2}^{2}\right)}{\left(1-\zeta^{2}\right) \zeta^{2}} .
\end{aligned}
$$

Partial differentiation with respect to $s, z$ and $r$ can be expressed as ${ }^{1}$

$$
\begin{aligned}
\left.\frac{\partial}{\partial s}\right|_{z} & =\left.\left.\frac{\partial x_{1}}{\partial s}\right|_{z} \frac{\partial}{\partial x_{1}}\right|_{x_{2}}+\left.\left.\frac{\partial x_{2}}{\partial s}\right|_{z} \frac{\partial}{\partial x_{2}}\right|_{x_{1}}=-\frac{\left(1-\zeta^{2}\right) s}{x_{1}^{2}-x_{2}^{2}}\left(x_{1} \frac{\partial}{\partial x_{1}}-x_{2} \frac{\partial}{\partial x_{2}}\right), \\
\left.\frac{\partial}{\partial z}\right|_{s} & =\left.\left.\frac{\partial x_{1}}{\partial z}\right|_{s} \frac{\partial}{\partial x_{1}}\right|_{x_{2}}+\left.\left.\frac{\partial x_{2}}{\partial z}\right|_{s} \frac{\partial}{\partial x_{2}}\right|_{x_{1}} \\
& =\frac{\zeta}{x_{1}^{2}-x_{2}^{2}}\left[x_{2}\left(x_{1}^{2}-1\right) \frac{\partial}{\partial x_{1}}-x_{1}\left(x_{2}^{2}-1\right) \frac{\partial}{\partial x_{2}}\right], \\
\left.\frac{\partial}{\partial r}\right|_{\theta} & =\left.\left.\frac{\partial z}{\partial r}\right|_{\theta} \frac{\partial}{\partial z}\right|_{s}+\frac{\partial s}{\partial r}\left|\frac{\partial}{\partial s}\right|_{z}=-\frac{\left(1-x_{1}^{2}\right) x_{1}}{\left(x_{1}^{2}-x_{2}^{2}\right) r} \frac{\partial}{\partial x_{1}}+\frac{\left(1-x_{2}^{2}\right) x_{2}}{\left(x_{1}^{2}-x_{2}^{2}\right) r} \frac{\partial}{\partial x_{2}} .
\end{aligned}
$$

We consider now density profiles with only radial dependence in spherical coordinates $(r, \theta, \varphi)$ in the form of a power law

$$
\rho=\tilde{\rho_{0}}\left(1-r^{2}\right)^{\beta}=\tilde{\rho_{0}}\left[\left(x_{1}-\zeta\right)^{2}\left(\zeta^{2}-x_{2}^{2}\right)\right]^{\beta} .
$$

The density scale height $H$ writes

$$
\begin{aligned}
H^{-1} \equiv-\frac{\mathrm{d} \ln \rho}{\mathrm{d} r} & =-\left(\frac{z}{r} \frac{\partial}{\partial z}+\frac{s}{r} \frac{\partial}{\partial s}\right) \ln \rho \\
& =2 \frac{\left(1-\zeta^{2}\right) \zeta^{2} \beta r}{\left(x_{1}^{2}-\zeta^{2}\right)\left(\zeta^{2}-x_{2}^{2}\right)} .
\end{aligned}
$$

\footnotetext{
${ }^{1}$ The original equation (A6) in Wu (2005a) is incorrect. The correct form is reproduced here.
} 
The partial differential equation (A.4) can be recast into

$$
\left[D_{1}+\frac{2 \beta x_{1}\left(1-x_{1}^{2}\right)}{x_{1}^{2}-\zeta^{2}} \frac{\partial}{\partial x_{1}}+\frac{2 \zeta \beta k}{x_{1}^{2}-\zeta^{2}}\right] \tilde{\psi}-\left[D_{2}+\frac{2 \beta x_{2}\left(1-x_{2}^{2}\right)}{x_{2}^{2}-\zeta^{2}} \frac{\partial}{\partial x_{2}}+\frac{2 \zeta \beta k}{x_{2}^{2}-\zeta^{2}}\right] \tilde{\psi}=0
$$

with

$$
D_{i}=\frac{\partial}{\partial x_{i}}\left[\left(1-x_{i}^{2}\right) \frac{\partial}{\partial x_{i}}\right]-\frac{k^{2}}{1-x_{i}^{2}} .
$$

Therefore, with the density profile in power law form the equation for the inertial modes are separable. With the decomposition $\tilde{\psi}\left(x_{1}, x_{2}\right)=\tilde{\psi}_{1}\left(x_{1}\right) \tilde{\psi}_{2}\left(x_{2}\right), \tilde{\psi}_{i}$ needs to satisfy

$$
\left[D_{i}+\frac{2 \beta x_{i}\left(1-x_{i}^{2}\right)}{x_{i}^{2}-\zeta^{2}} \frac{\partial}{\partial x_{i}}+\frac{2 \zeta \beta k}{x_{i}^{2}-\zeta^{2}}\right] \tilde{\psi}_{i}+K_{\mathrm{e}}^{2} \tilde{\psi}_{i}=0
$$

with $K_{\mathrm{e}}$ a constant introduced due to the separation of variables, we define $K_{\mathrm{e}}^{2} \equiv l_{\mathrm{e}}\left(l_{\mathrm{e}}+1\right)$. In the incompressible case it is $\tilde{\psi}_{1}=\tilde{\psi}_{2}=P_{n}^{k}$, with $P_{n}^{k}$ a spherical harmonic of the first kind and $n=l_{\mathrm{e}}$. As in $\mathrm{Wu}(2005 \mathrm{a})$ we now introduce a variable $g_{i}$ that is related to $\tilde{\psi}_{i}$ as

$$
\tilde{\psi}_{i}\left(x_{i}\right)=\left(1-x_{i}^{2}\right)^{|k| / 2} g_{i}\left(x_{i}\right)
$$

This variable satisfies

$$
\begin{array}{r}
\left(1-x_{i}^{2}\right) \frac{\mathrm{d}^{2} g_{i}}{\mathrm{~d} x_{i}^{2}}-2 x_{i}(|k|+1) \frac{\mathrm{d} g_{i}}{\mathrm{~d} x_{i}}+\frac{2 \beta x_{i}\left(1-x_{i}^{2}\right)}{x_{i}^{2}-\zeta^{2}} \frac{\mathrm{d} g_{i}}{\mathrm{~d} x_{i}} \\
+\left[o^{2}-\frac{2 \beta|k| x_{i}^{2}}{x_{i}^{2}-\zeta^{2}}+\frac{2 \beta k \zeta}{x_{i}^{2}-\zeta^{2}}\right] g_{i}=0
\end{array}
$$

where $o^{2}=K_{\mathrm{e}}^{2}-|k|(|k|+1)$. Due to this new variable the condition for regularity at $x_{1}=1$ can be written as a boundary condition

$$
\left.\frac{\mathrm{d} g_{1}}{\mathrm{~d} x_{1}}\right|_{x_{1}=1}=\frac{o^{2}+2 \beta[k \zeta-|k|] /\left(1-\zeta^{2}\right)}{2(|k|+1)} g_{1}
$$

Furthermore at the equator $\left(x_{2}=0\right)$, even parity modes satisfy

$$
\left.\frac{\mathrm{d} g_{2}}{\mathrm{~d} x_{2}}\right|_{x_{2}=0}=0
$$

while odd parity modes satisfy

$$
\left.g_{2}\right|_{x_{2}=0}=0 .
$$

As written in Sect. 2.1.1 at the surface $\left(x_{1}=\zeta\right.$ or $\left.\left|x_{2}\right|=\zeta\right) \xi_{r}=0$ is required. The radial 
displacement is related to $\tilde{\psi}$ as ${ }^{2}$

$$
\xi_{r}=\frac{\left(1-x_{1}^{2}\right)\left(1-x_{2}^{2}\right)}{\left(1-\zeta^{2}\right)\left(x_{1}^{2}-x_{2}^{2}\right) r}\left[x_{1} \frac{\partial \tilde{\psi}}{\partial x_{1}} \frac{\zeta^{2}-x_{2}^{2}}{1-x_{2}^{2}}-x_{2} \frac{\partial \tilde{\psi}}{\partial x_{2}} \frac{\zeta^{2}-x_{1}^{2}}{1-x_{1}^{2}}+k \zeta \frac{x_{1}^{2}-x_{2}^{2}}{\left(1-x_{1}^{2}\right)\left(1-x_{2}^{2}\right)} \tilde{\psi}\right]
$$

and therefore $\xi_{r}=0$ translates into

$$
\begin{gathered}
\left.\frac{\partial \tilde{\psi}_{1}}{\partial x_{1}}\right|_{x_{1}=\zeta}=-\left.\frac{k}{1-\zeta^{2}} \tilde{\psi}_{1}\right|_{x_{1}=\zeta}, \\
\left.\frac{\partial \tilde{\psi}_{2}}{\partial x_{2}}\right|_{\left|x_{2}\right|=\zeta}=-\left.\operatorname{SIGN}\left[x_{2}\right] \frac{k}{1-\zeta^{2}} \tilde{\psi}_{2}\right|_{\left|x_{2}\right|=\zeta}
\end{gathered}
$$

Equation (A.19) is modified for $g_{i}$ as

$$
\begin{gathered}
\left.\frac{\partial g_{1}}{\partial x_{1}}\right|_{x_{1}=\zeta}=\left.\frac{-(k-|k| \zeta)}{1-\zeta^{2}} g_{1}\right|_{x_{1}=\zeta}, \\
\left.\frac{\partial g_{2}}{\partial x_{2}}\right|_{\left|x_{2}\right|=\zeta}=-\left.\operatorname{SIGN}\left[x_{2}\right] \frac{k-|k| \zeta}{1-\zeta^{2}} g_{2}\right|_{\left|x_{2}\right|=\zeta}
\end{gathered}
$$

\footnotetext{
${ }^{2}$ The original equation $(28)$ in $\mathrm{Wu}(2005 \mathrm{a})$ is incorrect. The correct form is reproduced here.
} 


\section{B Orthogonality of the scalar prod- uct}

This proof follows the proceeding in Greenspan (1968). With $\left(\mathbf{u}_{i}, q_{i}\right),\left(\mathbf{u}_{j}, q_{j}\right)$ two arbitrary eigenfunction-eigenvalue pairs which satisfy Eq. (2.25) and $q_{i} \neq q_{j}$. It is

$$
\begin{array}{r}
\mathrm{i} q_{i} \rho_{0} \mathbf{u}_{j}^{\dagger} \cdot \mathbf{u}_{i}+2 \rho_{0} \mathbf{u}_{j}^{\dagger} \cdot \Lambda \mathbf{u}_{i}(\Omega+1)=-\rho_{0} \mathbf{u}_{j}^{\dagger} \cdot \nabla \psi_{i} \\
-\mathrm{i} q_{j} \rho_{0} \mathbf{u}_{i}^{\dagger} \cdot \mathbf{u}_{j}+2 \rho_{0} \mathbf{u}_{i}^{\dagger} \cdot \Lambda \mathbf{u}_{j}(\Omega+1)=-\rho_{0} \mathbf{u}_{i}^{\dagger} \cdot \nabla \psi_{j},
\end{array}
$$

deduced from Eq. (2.25), we used that $q_{i}=\omega_{i}+k_{i}$. It is $\boldsymbol{\Lambda} \mathbf{u}_{i}=\hat{\mathbf{z}} \times \mathbf{u}_{i}$, therefore, according to the rules for a scalar triple product $\mathbf{u}_{j}^{\dagger} \cdot \Lambda \mathbf{u}_{i}=-\mathbf{u}_{i} \cdot \Lambda \mathbf{u}_{j}^{\dagger}$. Now adding the two equations in (B.1) and integrating over the volume gives

$$
\left(q_{i}-q_{j}\right) \int \rho_{0} \mathbf{u}_{j}^{\dagger} \cdot \mathbf{u}_{i} d V=0
$$

According to the assumption $q_{i} \neq q_{j}$ and therefore $\int \rho_{0} \mathbf{u}_{j}^{\dagger} \cdot \mathbf{u}_{i} d V=0$. 


\section{Relation between frequency dif- ference and ellipticity}

For instability

$$
0<\sigma_{i n v}^{2}=-\frac{\left(2 W^{2} \pm \Delta \omega\right)^{2}+4 W^{2} q_{i} q_{j}}{4\left(1-W^{2}\right)^{2}}
$$

needs to be the case. If the right hand side is negative the flow is with safety stable. For all modes it is $\left|q_{i}\right| \leq 2$ and for all mode-pairs $0 \leq \tilde{W}^{2} \leq 1$, with $\tilde{W} \equiv W / \epsilon$. With this relations from Eq. (C.1) can be deduced that we need to find out for which $\Delta \omega$

$$
0 \geq-\left(2 W \pm \frac{\Delta \omega}{W}\right)^{2}+16
$$

without fail, to be sure that the flow is stable. We start by determining for which $W$ the term in brackets on the right hand side is maximal. The first derivative is

$$
0=\frac{\mathrm{d}}{\mathrm{d} W}\left(2 W \pm \frac{\Delta \omega}{W}\right)^{2}=2\left(2 W \pm \frac{\Delta \omega}{W}\right)\left(2 \mp \frac{\Delta \omega}{W^{2}}\right)
$$

a check of the second derivative

$$
0=\frac{\mathrm{d}^{2}}{\mathrm{~d} W^{2}}\left(2 W \pm \frac{\Delta \omega}{W}\right)^{2}=-\frac{2\left(4 W^{4}+2 \Delta \omega^{2}\right)}{W^{4}}
$$

gives that the maximum lies at $\tilde{W}_{\max }= \pm \sqrt{|\Delta \omega| /\left(2 \epsilon^{2}\right)}$. We distinguish between two cases

- $|\Delta \omega| \geq 2 \epsilon^{2}$ : For this case it is $\tilde{W}_{\max }^{2} \geq 1$, it needs to be $\tilde{W}^{2} \leq 1$, because of the normalization of the inertial modes. The second derivative Eq. (C.4) is negative everywhere, therefore, the maximum value of this term with the constraint $\tilde{W}^{2} \leq 1$ is at $\tilde{W}= \pm 1$. For $\tilde{W}=1$ (C.2) gives that if $|\Delta \omega| \geq 4 \epsilon+2 \epsilon^{2}$ the flow is stable.

- $|\Delta \omega|<2 \epsilon^{2}$ : This case occurs for $|\Delta \omega|<2 \epsilon^{2}$. 
We can conclude that only for

$$
|\Delta \omega| \leq 4 \epsilon+2 \epsilon^{2}
$$

instability is possible. 


\section{Equations governing spheroidal oscillations}

This derivation follows the proceeding in Sabadini and Vermeersen (2004). The following formulations are expressed in spherical coordinates $(r, \theta, \varphi)$. The spherical coordinates can be chosen such that the problem is rotationally symmetric, see Eq. (3.2). We will make use of this simplification in the following, such that the longitudinal component drops out and the solution has to be only expanded in Legendre polynomials. Taking account of the continuity equation, $\nabla \cdot \boldsymbol{\xi}=0$, the $r$ and $\theta$ component of the momentum equation and the Poisson equation become

$$
\begin{aligned}
-\rho \partial_{r} \Phi^{\prime}-\rho \partial_{r}\left(\xi_{r} g\right)+\partial_{r} \sigma_{r r}+r^{-1} \partial_{\theta} \sigma_{r \theta}+r^{-1}\left(2 \sigma_{r r}-\sigma_{\theta \theta}-\sigma_{\varphi \varphi}+\sigma_{r \theta} \cot \theta\right) & =0 \\
-\rho r^{-1} \partial_{\theta} \Phi^{\prime}-\rho g r^{-1} \partial_{\theta} \xi_{r}+\partial_{r} \sigma_{r \theta}+r^{-1} \partial_{\theta} \sigma_{\theta \theta}+r^{-1}\left(\left(\sigma_{\theta \theta}-\sigma_{\varphi \varphi}\right) \cot \theta+3 \sigma_{r \theta}\right) & =0 \\
r^{-2} \partial_{r}\left(r^{2} \partial_{r} \Phi^{\prime}\right)+\left(r^{2} \sin \theta\right)^{-1} \partial \theta\left(\sin \theta \partial_{\theta} \Phi^{\prime}\right) & =0
\end{aligned}
$$

where the $\sigma_{i j}$ 's denote the stress components in spherical coordinates and $\xi_{r}$ the radial component of the displacement vector. In this appendix we omit the prime about the perturbation quantities $\sigma_{i j}$ because only the perturbation appears here, and we omit the tilde above the complex quantities because the equations here are valid for complex $\mu$ according to the viscoelastic problem as well as for real $\mu$ according to the pure elastic problem. For the stress strain relations, it is assumed that materials in the planet obey a generalized Hooke's law, this means it exists a linear dependence between stress and strain. The components of stress tensor are

$$
\begin{aligned}
\sigma_{\theta \theta} & =\lambda \nabla \cdot \boldsymbol{\xi}+2 \mu \epsilon_{\theta \theta}, \\
\sigma_{\varphi \varphi} & =\lambda \nabla \cdot \boldsymbol{\xi}+2 \mu \epsilon_{\varphi \varphi}, \\
\sigma_{r \theta} & =2 \mu \epsilon_{r \theta} .
\end{aligned}
$$


Here the approximation of incompressibility will be used, therefore, $\lambda$ Lame's second parameter goes to infinity while the dilatation $\nabla \cdot \boldsymbol{\xi}$ goes to zero and the product $\Pi=\lambda \nabla \cdot \boldsymbol{\xi}$ remains finite. A formulation for $\Pi$ can be found e.g. in Henning and Hurford (2014), it is not stated here, because in the final equations to solve it doesn't appear. The components of the strain tensor are

$$
\begin{array}{r}
\epsilon_{r r}=\partial_{r} \xi_{r}, \\
\epsilon_{\theta \theta}=\frac{1}{r}\left(\partial_{\theta} \xi_{\theta}+\xi_{r}\right), \\
\epsilon_{\varphi \varphi}=\frac{1}{r}\left(\xi_{\theta} \cot \theta+\xi_{r}\right), \\
\epsilon_{r \theta}=\frac{1}{2}\left(\partial_{r} \xi_{\theta}-\frac{\xi_{\theta}}{r}+\frac{\partial_{\theta} \xi_{r}}{r}\right),
\end{array}
$$

with $\xi_{\theta}$ the tangential component of the displacement vector. The $\mathrm{r}$ component of the momentum equations becomes in terms of the displacement components

$$
\begin{array}{r}
-\rho \partial_{r} \Phi^{\prime}-\rho \partial_{r}\left(\xi_{r} g\right)+\partial_{r}\left(\Pi+2 \mu \partial_{r} \xi_{r}\right) \\
+\frac{\mu}{r^{2}}\left[4 r \partial_{r} \xi_{r}-4 \xi_{r}+r\left(\partial_{\theta} \partial_{r} \xi_{\theta}+\partial_{r} v \cot \theta\right)\right. \\
\left.+\partial_{\theta}^{2} \xi_{r}+\partial_{\theta} \xi_{r} \cot \theta-3\left(\partial_{\theta} \xi_{\theta}+\xi_{\theta} \cot \theta\right)\right]=0 .
\end{array}
$$

The $\theta$ component of the momentum equation is given by

$$
\begin{array}{r}
-\frac{\rho}{r} \partial_{\theta} \Phi^{\prime}-\frac{\rho g}{r} \partial_{\theta} \xi_{r}+\mu \partial_{r}\left(\partial_{r} \xi_{\theta}-\frac{\xi_{\theta}}{r}+\frac{1}{r} \partial_{\theta} \xi_{r}\right) \\
+\frac{1}{r} \partial_{\theta} \Pi+\frac{2 \mu}{r^{2}}\left(\partial_{\theta}^{2} v_{\theta}+\partial_{\theta} \xi_{\theta} \cot \theta-\xi_{\theta} \cot ^{2} \theta-\xi_{\theta}\right) \\
+\frac{3 \mu}{r} \partial_{r} \xi_{\theta}+\frac{5 \mu}{r^{2}} \partial_{\theta} \xi_{r}-\frac{\mu}{r^{2}} \xi_{\theta}=0 .
\end{array}
$$

The divergence of the displacement in spherical coordinates is

$$
\nabla \cdot \boldsymbol{\xi}=\partial_{r} \xi_{r}+\frac{2}{r} \xi_{r}+\frac{2}{r} \partial_{\theta} \xi_{\theta}+\frac{\cot \theta}{r} \xi_{\theta}
$$

Solutions of the form

$$
\begin{aligned}
\xi_{r} & =\sum_{l=0}^{\infty} y_{1, l}(r) P_{l}(\cos \theta), \\
\xi_{\theta} & =\sum_{l=0}^{\infty} y_{3, l}(r) \partial_{\theta} P_{l}(\cos \theta), \\
\Phi^{\prime} & =\sum_{l=0}^{\infty} y_{5, l}(r) P_{l}(\cos \theta),
\end{aligned}
$$

are assumed for the spheroidal oscillations, where $y_{i, l}(r)$ is an unknown function to be determined. Only the $y_{i, 2}(r)$ are nonzero for the tidal problem. Henceforth, we omit the subscript $l$ at the $y_{i, l}$. 
Now with the Legendre equation

$$
\frac{\mathrm{d}^{2}}{\mathrm{~d} \theta^{2}} P_{l}(\theta)+\cot \theta \frac{\mathrm{d}}{\mathrm{d} \theta} P_{l}(\theta)=-l(l+1) P_{l}(\theta)
$$

and the derivative of the Legendre equation

$$
\frac{\mathrm{d}^{3}}{\mathrm{~d} \theta^{3}} P_{l}(\theta)+\frac{\mathrm{d}^{2}}{\mathrm{~d} \theta^{2}} P_{l}(\theta) \cot \theta-\frac{\mathrm{d}}{\mathrm{d} \theta} P_{l}(\theta)\left[1+\cot ^{2} \theta\right]=-l(l+1) \frac{\mathrm{d}}{\mathrm{d} \theta} P_{l}(\theta),
$$

the $r$ and $\theta$ components of the momentum equations become

$$
\begin{array}{r}
\rho \partial_{r} \varphi_{l}-\rho \partial_{r}\left(g y_{1}\right)+\partial_{r}\left(\Pi+2 \mu \partial_{r} y_{1}\right)+\frac{\mu}{r^{2}}\left[4 r \partial_{r} y_{1}-4 y_{1}+l(l+1)\left(-y_{1}-r \partial_{r} y_{3}+3 y_{3}\right)\right]=0, \\
\text { (D.10a) } \\
\rho y_{5}-\rho g y_{1}+\Pi+\mu r \partial_{r}\left(\partial_{r} y_{3}-\frac{y_{3}}{r}+\frac{y_{1}}{r}\right)+\frac{\mu}{r}\left[5 y_{1}+3 r \partial_{r} y_{3}-y_{3}-2 l(l+1) y_{3}\right]=0 .
\end{array}
$$

The Laplace equation can be written as

$$
\partial_{r}^{2} y_{5}+\frac{2}{r} \partial_{r} y_{5}-\frac{l(l+1)}{r^{2}} y_{5}=0
$$

and the continuity equation as

$$
0=\partial_{r} y_{1}+\frac{2}{r} y_{1}-\frac{l(l+1)}{r} y_{3} .
$$

With the definitions

$$
\begin{array}{r}
y_{2}=\Pi+2 \mu \partial_{r} y_{1}, \\
y_{4}=\mu\left(\partial_{r} y_{3}-\frac{y_{3}}{r}+\frac{y_{1}}{r}\right), \\
y_{6}=\frac{\mathrm{d} y_{5}}{\mathrm{~d} r}-4 \pi G \rho y_{1}+\frac{l+1}{r} y_{5} .
\end{array}
$$

The momentum and Laplace equation for the incompressible case can now be written in the form of Eqs. (3.11). $y_{2}$ and $y_{4}$ are associated with radial and tangential stresses, respectively, the meaning of $y_{6}$ is depicted in Appendix E. 


\section{E Continuity of the total gravita- tional potential and its gradient}

Except at liquid solid boundaries displacement, stress, and potential have to be continuous, this condition is automatically satisfied if the functions $y_{i}(i=1,2, . ., 5)$ are continuous. Another condition is that

$$
\frac{\mathrm{d} y_{5}}{\mathrm{~d} r}-4 \pi G \rho y_{1}+\frac{l+1}{r} y_{5}=\text { continuous. }
$$

It will now be explained why this has to be the case. The explanation here is analogue to the proceeding in Saito (1974). Deformation due to a tidal potential of the form

$$
\Phi_{t}^{\prime}=\left(\frac{r}{R_{\mathrm{p}}}\right)^{l} Y_{l}(\theta, \varphi)
$$

is considered. The planet undergoes redistribution of mass due to this potential, which in turn changes its gravitational potential. The total perturbation in potential can then be written as $\Phi^{\prime}=\Phi_{t}^{\prime}+\Phi_{d}^{\prime}$, with $\Phi_{d}^{\prime}$ the perturbation in gravitational potential due to the mass redistribution. It is assumed that outside the planet

$$
\Phi_{d}^{\prime}=k_{l}\left(\frac{R_{\mathrm{p}}}{r}\right)^{l+1} Y_{l}(\theta, \varphi)
$$

with $k_{l}$ a tidal Love number (see Eqs. 3.21). The gradient of total potential has to be continuous, therefore,

$$
\begin{array}{r}
\frac{\partial \Phi_{0}\left(\mathbf{r}_{0}+\boldsymbol{\xi}\right)}{\partial r}+\frac{\partial \Phi\left(\mathbf{r}_{0}+\boldsymbol{\xi}\right)}{\partial r} \\
=\frac{\partial \Phi_{0}\left(\mathbf{r}_{0}\right)}{\partial r}+\xi_{r}\left(\frac{\partial^{2} \Phi_{0}\left(\mathbf{r}_{0}\right)}{\partial r^{2}}+4 \pi G \rho_{0}\right)+\left(\frac{\partial \Phi\left(\mathbf{r}_{0}\right)}{\partial r}-4 \pi G \rho_{0} \xi_{r}\right) .
\end{array}
$$


The first and the second term are always continuous, the first because it represents the $\mathrm{r}$ component of gravity and the second because it is the Poisson equation for the undisturbed spherical planet. We can deduce that

$$
\frac{\partial \Phi\left(\mathbf{r}_{0}\right)}{\partial r}-4 \pi G \rho_{0} \xi_{r}=\text { continous }
$$

at any surface of discontinuity. Therefore, across the surface of the planet

$$
\left(\frac{\partial \Phi_{d}^{\prime}}{\partial r}-4 \pi G \rho \xi_{r}\right)_{i}=\left(\frac{\partial \Phi_{d}^{\prime}}{\partial r}\right)_{e}
$$

with $i$ and $e$ referring to values just inside and outside the planet's surface, respectively. With $(\Phi)_{i}=y_{5}(r) Y_{l}(\theta, \varphi)$ and Eqs. E.2 and E.3 one surface boundary condition is

$$
\frac{\mathrm{d} y_{5}}{\mathrm{~d} r}-4 \pi G \rho y_{1}+\frac{l+1}{R_{p}} y_{5}=\frac{2 l+1}{R_{p}} .
$$

This is not automatically satisfied, even if the $y_{i}(i=1,2, . ., 5)$ are continuous, but with the definition $y_{6}=\frac{\mathrm{d} y_{5}}{\mathrm{~d} r}-4 \pi G \rho y_{1}+\frac{l+1}{r} y_{5}$ and the boundary condition $y_{6}=(2 l+1) / R_{p}$ at the surface, it will be satisfied. 


\section{F MAPLE commands}

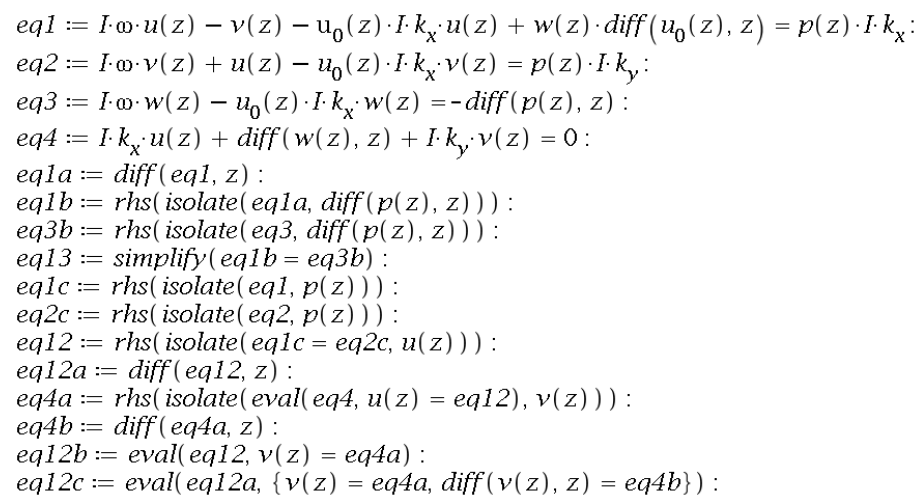

(a)

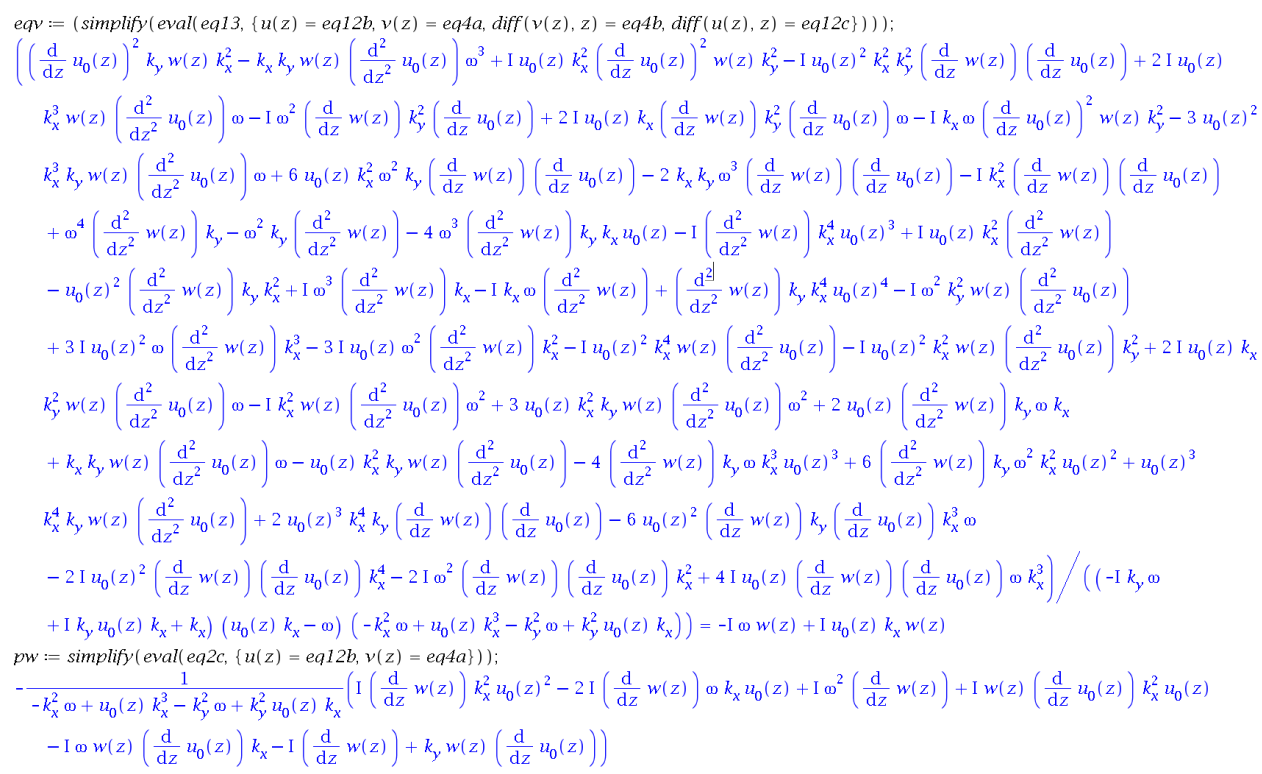

Figure F.1. 


\section{Bibliography}

M. Abramowitz and I. A. Stegun. Handbook of Mathematical Functions. Dover Publications, New York, 1972.

Guenter Ahlers, Siegfried Grossmann, and Detlef Lohse. Heat transfer and large scale dynamics in turbulent Rayleigh-Bénard convection. Rev. Mod. Phys., 81:503-537, Apr 2009 .

K. Aldridge, B. Seyed-Mahmoud, G. Henderson, and W. van Wijngaarden. Elliptical instability of the Earth's fluid core. Phys. Earth Planet. Inter., 103:365 - 374, 1997.

Z. Alterman, H. Jarosch, and C. Pekeris. Oscillations of the Earth. Proc R. Soc. London A, 252:80 - 95, 1959 .

B. A. Archinal, M. F. A'Hearn, E. Bowell, et al. Report of the IAU working group on cartographic coordinates and rotational elements: 2009. Celest. Mech. Dyn. Astron., 109:101-135, 2011.

A. J. Barker and Y. Lithwick. Non-linear evolution of the tidal elliptical instability in gaseous planets and stars. MNRAS, 435:3614-3626, 2013a.

A. J. Barker and Y. Lithwick. Non-linear evolution of the elliptical instability in the presence of weak magnetic fields. MNRAS, 437:305-315, 2014.

A. J. Barker, A. M. Dempsey, and Y. Lithwick. Theory and simulations of rotating convection. ApJ, 791(1):13, 2014.

B. J. Bayly. Three-dimensional instability of elliptical flow. Phys. Rev. Lett., 57:2160-2163, 1986.

M. Behounkova, G. Tobie, G. Choblet, and O. Cadek. Impact of tidal heating on the onset of convection in Enceladus's ice shell. Icarus, 226(1):898 - 904, 2013. 
M. Beuthe. Spatial patterns of tidal heating. Icarus, 223(1):308 - 329, 2013.

M. A. Biot. Theory of stress-strain relations in anisotropic viscoelaticity and relaxation phenomena. J. Appl. Phys, 25:1385-1391, 1954.

G. H. Bryan. The waves on a rotating liquid spheroid of finite ellipticity. Phil. Trans. R. Soc. London Ser. A, 180:187-219, 1889.

M. H. Carr, M. J. S. Belton, C. R. Chapman, et al. Evidence for a subsurface ocean on Europa. Nature, 391:363-365, 1998.

D. Cébron, M. Le Bars, J. Leontini, P. Maubert, and P. Le Gal. A systematic numerical study of the tidal instability in a rotating triaxial ellipsoid. Phys. Eart Planet. Inter., 182:119-128, 2010a.

D. Cébron, P. Maubert, and M. Le Bars. Tidal instability in a rotating and differentially heated ellipsoidal shell. Geophys. J. Int., 182:1311-1318, 2010b.

D. Cébron, M. Le Bars, C. Moutou, and P. Le Gal. Elliptical instability in terrestrial planets and moons. A\&SA, 539:A78, 2012.

D. Cébron, M. Le Bars, P. Le Gal, C. Moutou, J. Leconte, and A. Sauret. Elliptical instability in hot Jupiter systems. Icarus, 226:1642-1653, 2013.

D. Cébron, S. Vantieghem, and W. Herreman. Libration-driven multipolar instabilities. JFM, 739:502-543, 2014.

G. Chabrier, I. Baraffe, J. Leconte, J. Gallardo, and T. Barman. The massradius relationship from solartype stars to terrestrial planets: A review. AIP Conf. Proc., 1094: 102-111, 2009.

A. Chu, W. Paech, and M. Weigand. The Cambridge photographic moon atlas. Cambridge University Press, Cambridge, 2012.

J. V. Clausen, H. Bruntt, A. Claret, et al. Absolute dimensions of solar-type eclipsing binaries. II. V636 Centauri: A 1.05 Msun primary with an active, cool oversize 0.85 Msun secondary. A\&\&A, 502:253-265, 2009.

N. Clausen and A. Tilgner. Elliptical instability of compressible flow in ellipsoids. $A \mathscr{G} A$, 562:A25, 2014. 
N. Clausen and A. Tilgner. Dissipation in rocky planets for strong tidal forcing. $A \mathscr{E} A$, 584:A60, 2015.

P. Colwell. Bessel functions and Kepler's equation. The American Mathematical Monthly, 99:45-48, 1992.

A. D. D. Craik. The stability of unbounded two- and three-dimensional flows subject to body forces: some exact solutions. JFM, 198:275-292, 1989.

F. A. Dahlen and J. Tromp. Theoretical Global Seismology. Princeton University Press, Princeton, 1998.

G. H. Darwin. On the bodily tides of viscous and semi-elastic spheroids, and on the ocean tides upon a yielding nucleus. Phil. Trans. R. Soc. London, 170:1-35, 1879.

F. Deschamps and J.-R. Lin. Stagnant lid convection in 3d-cartesian geometry: Scaling laws and applications to icy moons and dwarf planets. Phys. Earth Planet. Inter., 229 (0):40 - 54, 2014.

G. A. DeWijs, G. Kresse, L. Vocadlo, et al. The viscosity of liquid iron at the physical conditions of the Earth's core. Nature, 392:805-807, 1998.

A. P. Douce. Thermodynamics of the Earth and Planets. Cambridge University Press, Cambridge, 2011.

C. Dumoulin, M.-P. Doin, and L. Fleitout. Heat transport in stagnant lid convection with temperature- and pressure-dependent newtonian or non-newtonian rheology. J. Geophys. Res.: Solid Earth, 104(B6):12759-12777, 1999.

M. Efroimsky. Tidal dissipation compared to seismic dissipation: In small bodies, earths, and super-earths. ApJ, 746(2):150, 2012.

M. Efroimsky and V. Lainey. Physics of bodily tides in terrestrial planets and the appropriate scales of dynamical evolution. J. Geophys. Res.: Planets, 112(E12), 2007.

C. Eloy, P. Le Gal, and S. Le Dizès. Elliptic and triangular instabilities in rotating cylinders. JFM, 476:357-388, 2003.

B. R. Fabijonas and D. D. Holm. Mean effects of turbulence on elliptic instability in fluids. Phys. Rev. Lett., 90:124501(1)-124501(4), 2003. 
H.-J. Fischer and T. Spohn. Thermal-orbital histories of viscoelastic models of Io. Icarus, 83(1):39-65, 1990.

M. S. Ghiorso and R. O. Sack. Chemical mass transfer in magmatic processes IV. A revised and internally consistent thermodynamic model for the interpolation and extrapolation of liquid-solid equilibria in magmatic systems at elevated temperatures and pressures. Contrib. Mineral. Petrol., 119(2-3):197-212, 1995.

E. B. Gledzer and V. M. Ponomarev. Finite-dimensional approximation of the motions of an incompressible fluid in an ellipsoidal cavity. Izvestiya Atmospheric and Oceanic Physics, 13:565-569, 1977.

E. B. Gledzer and V. M. Ponomarev. Instability of bounded flows with elliptical streamlines. JFM, 240:1-30, 1992.

E. B. Gledzer, F. V. Dolzhansky, A. M. Obhukov, and V. M. Ponomarev. An experimental and theoretical study of the stability of motion of a liquid in an elliptical cylinder. Izvestiya Atmospheric and Oceanic Physics, 11:617-622, 1975.

P. Goldreich and P. D. Nicholson. Turbulent viscosity and Jupiter's tidal Q. Icarus, 30: 301-304, 1977.

P. Goldreich and S. Soter. Q in the solar system. Icarus, 5:375 - 389, 1966.

H. P. Greenspan. The Theory of rotating Fluids. Cambridge University Press, Cambridge, 1968.

T. Guillot, D. J. Stevenson, W. B. Hubbard, and D. Saumon. The interior of Jupiter. In Jupiter: The Planet, Satellites and Magnetosphere, pages 35-57. Bagenal, F. Dowling, D. and McKinnon, W., 2004.

D. Guimbard, S. Le Dizès, M. Le Bars, P. Le Gal, and S. Leblanc. Elliptic instability of a stratified fluid in a rotating cylinder. JFM, 660:240-257, 2010.

B. M. S. Hansen and N. Murray. Testing in situ assembly with the Kepler planet candidate sample. ApJ, 775(1), 2013.

B. M. S. Hansen and N. Murray. Secular effects of tidal damping in compact planetary systems. MNRAS, 448:1044-1059, 2015.

C. Hellier, D. R. Anderson, A. Collier Cameron, et al. An orbital period of 0.94 days for the hot-Jupiter planet WASP-18b. Nature, 460:1098-1100, 2009. 
W. G. Henning and T. Hurford. Tidal heating in multilayered terrestrial exoplanets. ApJ, 789(1):30, 2014.

W. G. Henning, R. J. O'Connell, and D. D. Sasselov. Tidally heated terrestrial exoplanets: viscoelastic response models. ApJ, 707(17):1000-1015, 2009.

W. Herreman, M. Le Bars, and P. Le Gal. On the effects of an imposed magnetic field on the elliptical instability in rotating spheroids. Phys. Fluids, 21(4):046602-1-046602-9, 2009 .

G. P. Horedt. Seven-Digit tables of Lane-Emden functions. Ap\& SS, 126:357-408, 1986.

G. P. Horedt. Polytropes. Kluwer Academic Publisher, Dordrecht Boston London, 2004.

U. Ingard. Influence of fluid motion past a plane boundary on sound reflection, absorption, and transmission. J. Acoust. Soc. Am., 31(7):1035-1036, 1959.

P. B. Ivanov and J. C. B. Papaloizou. Inertial waves in rotating bodies: A WKBJ formalism for inertial modes and a comparison with numerical results. MNRAS, 407:1609-1630, 2010 .

B. Jackson, R. Greenberg, and R. Barnes. Tidal evolution of close-in extrasolar planets. ApJ, 678(2):1396, 2008.

R. A. Jacobson. Jup230. JPL satellite ephemeris, 2003.

S. Karato. Physics and Chemistry of the Deep Earth. Wiley-Blackwell, 2013.

R. R. Kerswell. The instability of precessing flow. Geophys. Astrophys. Fluid Dyn., 72: 107-144, 1993.

R. R. Kerswell. Tidal excitation of hydromagnetic waves and their damping in the Earth. JFM, 274:219-241, 1994.

R. R. Kerswell. Elliptical instability. Ann. Rev. Fluid Mech., 34:83-113, 2002.

R. R. Kerswell and W. V. R. Malkus. Tidal instability as the source for Io's magnetic signature. Geophys. Res. Lett., 274:603-606, 1998.

L. Keszthelyi, W. L. Jaeger, E. P. Turtle, M. Milazzo, and J. Radebaugh. A post-Galileo view of Io's interior. Icarus, 169(1):271 - 286, 2004. Special Issue: Io after Galileo. 
K. K. Khurana, X. Jia, M. G. Kivelson, et al. Evidence of a global magma ocean in Io's interior. Science, 332(6034):1186-1189, 2011.

R. Kippenhahn and A. Weigert. Stellar Structure and Evolution. Springer Verlag, Berlin Heidelberg, 1990.

D. L. Kohlstedt and S. J. Mackwell. Strength and deformation of planetary lithospheres. In T. R. Watters and R. A. Schultz, editors, Planetary Tectonics, pages 397-456. Cambridge University Press, 2009.

L. Lacaze, P. Le Gal, and S. Le Dizès. Elliptical instability in a rotating spheroid. JFM, 505:1-22, 2004.

L. Lacaze, W. Herreman, M. Le Bars, S. Le Dizès, and P. Le Gal. Magnetic field induced by elliptical instability in a rotating spheroid. Geophys. Astrophys. Fluid Dyn., 100: 299-317, 2006.

V. Lainey, J.-E. Arlot, O. Karatekin, and T. Van Hoolst. Strong tidal dissipation in Io and Jupiter from astrometric observations. Nature, 459:957-959, 2009.

M. J. Landman and P. G. Saffman. The three-dimensional instability of strained vortices in a viscous fluid. Phys. Fluids, 30:2339-2342, 1987.

M. Le Bars, L. Lacaze, S. Le Dizès, P. Le Gal, and M. Rieutord. Tidal instability in stellar and planetary binary systems. Phys. Earth Planet. Inter., 178:48-55, 2010.

J. Leconte, G. Chabrier, I. Baraffe, and B. Levrard. Is tidal heating sufficient to explain bloated exoplanets? Consistent calculations accounting for finite initial eccentricity. A $B A$, 516:A64, 2010.

A. Léger, O. Grasset, B. Fegley, et al. The extreme physical properties of the CoRoT-7b super-Earth. Icarus, 213(1):1 - 11, 2011.

S. Lorenzani. Fluid instabilities in precessing ellipsoidal shells. $\mathrm{PhD}$ thesis, University of Göttingen, 2001.

A. E. H. Love. Some problems of geodynamics. Cambridge University Press, London and New York, 1911.

W. V. R. Malkus. An experimental study of global instabilities due to tidal (elliptical) distortion of a rotating elastic cylinder. Geophys. Astrophys. Fluid Dyn., 48:123-134, 1989 
S. Mathis, P. Auclair-Desrotour, M. Guenel, and C Le Poncin-Lafitte. Tidal friction in rotating turbulent convectivestellar and planetary regions. In J. Ballet, F. Martins, F. Bournaud, R. Monier, and C. Reylé, editors, SF2A-2014: Proceedings of the Annual meeting of the French Society of Astronomy and Astrophysics, pages 251-256, 2014.

S. Mei, W. Bai, T. Hiraga, and D. L. Kohlstedt. Influence of melt on the creep behavior of olivine-basalt aggregates under hydrous conditions. Earth Planet. Sci. Lett., 201:491 $-507,2002$.

S. Meibom and R. D. Mathieu. A robust measure of tidal circularization in coeval binary populations: The solar-type spectroscopic binary population in the open cluster M35. ApJ, 620(2):970, 2005.

M. S. Miesch. Large-scale dynamics of the convection zone and tachocline. Liv. Rev. Sol. Phys., 2:1-139, 2005.

J. W. Miles. On the reflection of sound at an interface of relative motion. J. Acoust. Soc. Am., 29:226-228, 1957.

K. J. Miller, W. Zhu, L. G. J. Montési, and G. A. Gaetani. Experimental quantification of permeability of partially molten mantle rock. Earth Planet. Sci. Lett., 388(0):273 282,2014 .

T. Miyazaki. Elliptical instability in a stably stratified rotating fluid. Phys. Fluids A: Fluid Dyn., 5:2702-2709, 1993.

T. Miyazaki and Y. Fukumoto. Three-dimensional instability of strained vortices in a stably stratified fluid. Phys.Fluids A: Fluid Dyn., 4:2515-2522, 1992.

T. Miyazaki, T. Imai, and Y. Fukumoto. Three-dimensional instability of Kirchhoff's elliptic vortex. Phys. Fluids, 7:195-202, 1995.

W. B. Moore. The thermal state of Io. Icarus, 154(2):548 - 550, 2001.

W. B. Moore. Tidal heating and convection in Io. J. Geophys. Res., 108(E8), 2003.

W. B. Moore, G. Schubert, J. D. Anderson, and J. R. Spencer. The interior of Io. In R.M.C. Lopez and J.R. Spencer, editors, Io after Galileo: A new view of Jupiter's volcanic moon, pages 89-105. Springer, 2007.

C. D. Murray and S. F. Dermott. Solar System Dynamics. Cambridge University Press, Cambridge, 1999. 
R. J. O'Connell and B. H. Hager. Physics of the Earth's interior. In Europa, pages 270-317.

Dziewonski, A.M. and Boschi, E., North Holland, 1980.

G. I. Ogilvie and G. Lesur. On the interaction between tides and convection. MNRAS, 422:1975-1987, 2012.

G. I. Ogilvie and D. N. C. Lin. Tidal dissipation in rotating solar-type stars. ApJ, 661: 1180-1191, 2007.

T. C. O'Reilly and G. F. Davies. Magma transport of heat on Io: A mechanism allowing a thick lithosphere. Geophys. Res. Lett., 8(4):313-316, 1981.

K. Penev and D. Sasselov. Tidal Evolution of Close-in Extrasolar Planets: High Stellar Q from New Theoretical Models. ApJ, 731(1):67, 2011.

K. Penev, J. Baranco, and D. Sasselov. Direct calculation of the turbulent dissipation efficiency in anelastic convection. ApJ, 705:285-297, 2009.

K. Penev, B. Jackson, F. Spada, and N Thom. Constraining tidal dissipation in stars from the destruction rates of exoplanets. ApJ, 751(2):96, 2012.

W. H. Press, S. A. Teukolsky, W. T. Vetterling, and B. P. Flannery. Numerical Recipes in Fortran 77. Cambridge University Press, Cambridge, 1992.

J. A. Rathbun, J. R. Spencer, L. K. Tamppari, et al. Mapping of Io's thermal radiation by the Galileo photopolarimeter-radiometer (PPR) instrument . Icarus, 169(1):127 139, 2004. Special Issue: Io after Galileo.

C. C. Reese, V. S. Solomatov, J. R. Baumgardner, and W.-S. Yang. Stagnant lid convection in a spherical shell. Phys. Earth Planet. Inter., 116:1-7, 1999.

C. C. Reese, V. S. Solomatov, and J. R. Baumgardner. Scaling laws for time-dependent stagnant lid convection in a spherical shell. Phys. Earth Planet. Inter., 149:361 - 370, 2005 .

H.S. Ribner. Reflection, transmission, and amplification of sound by a moving medium. J. Acoust. Soc. Am., 29:435-441, 1957.

M. Rieutord. Ekman circulation and synchronization of binary stars. A\&SA, 259:581-584, 1992. 
M. Rieutord. Evolution of rotation in binaries. IAU Symp. 215 "Stellar Rotation", ed. P. Eenes and A.Maeder, 2003.

M. Rieutord. The dynamics of rotating fluids and binary stars. EAS Publications series, 29:127-147, 2008.

M. Rieutord and J.-P. Zahn. Ekman pumping and tidal dissipation in close binaries: A refutation of Tassoul's mechanism. ApJ, 474:760-797, 1997.

A. Rodríguez, S. Ferraz-Mello, T. A. Michtchenko, C. Beaugé, and O. Miloni. Tidal decay and orbital circularization in close-in two-planet systems. MNRAS, 415(3):2349-2358, 2011.

R. Sabadini and B. Vermeersen. Global Dynamics of the Earth. Kluwer Academic Publishers, Dordrecht, 2004.

M. Saito. Some problems of static deformation of the Earth. J. Phys. Earth, 22:123-140, 1974.

G. Schubert, J. D. Anderson, T. Spohn, and W. B. McKinnon. Interior Composition, Structure and Dynamics of the Galilean Satellites. In Jupiter: The Planet, Satellites and Magnetosphere, pages 281-306. Bagenal, F. Dowling, T.E. and McKinnon, W.B., 2004 .

T. Scott and D.L. Kohlstedt. The effect of large melt fraction on the deformation behavior of peridotite. Earth Planet. Sci. Lett., 246(3?4):177 - 187, 2006.

M. Segatz, T. Spohn, M. N. Ross, and G. Schubert. Tidal dissipation, surface heat flow, and figure of viscoelastic models of Io. Icarus, 75(2):187 - 206, 1988.

B. Seyed-Mahmoud, G. Henderson, and K. Aldridge. A numerical model for the elliptical instability of the Earth's fluid outer core. Phys. Earth Planet. Inter., 117:51-61, 2000.

L. Shi, B. Hof, and A. Tilgner. Transient growth of Ekman-Couette flow. Phys. Rev. E, 89:013001, Jan 2014.

C. Sotin, G. Tobie, J. Wahr, and W. B. McKinnon. Tides and Tidal heating on Europa. In Pappalardo R. et al., editor, Europa, volume 11. University of Arizona Space Science Series, 2009. 
F. D. Stacey. Mantle, thermal conductivity. In D. Gubbins and E. Herrero-Bervera, editors, Encyclopedia of Geomagnetism and Paleomagnetism, pages 688-689. Springer Netherlands, 2007.

V. Stamenkovic and D. Breuer. The tectonic mode of rocky planets: Part 1 - driving factors, models \& parameters. Icarus, 234(0):174 - 193, 2014.

D. J. Stevenson. Turbulent thermal convection in the presence of rotation and a magnetic field: A heuristic theory. Geophys. Astrophys. Fluid Dyn., 12:139, 1979.

D. J. Stevenson. Tidal response and stability of two-phase media: Implications for Io, Europa and Titan. In Eos. Trans. AGU 83. P12C-10, 2002.

P.J. Tackley, M. Ammann, J.P. Brodholt, D.P. Dobson, and D. Valencia. Mantle dynamics in super-earths: Post-perovskite rheology and self-regulation of viscosity. Icarus, 225 (1):50 - 61, 2013.

H. Takeushi and M. Saito. Seismic surface waves. In B.A. Bolt, editor, Methods in computational physics, volume 11. Academic Press, New York, 1972.

J.-L. Tassoul. On synchronization in early type binaries. ApJ, 322:856-861, 1987.

J.-L. Tassoul. On orbital circularization in late-type close binaries. ApJ, 444:338-341, 1995.

M. Tassoul and J.-L. Tassoul. On synchronization in detached close binaries: Reply to Rieutord and Zahn. ApJ, 481:363-368, 1997.

G. Tobie, A. Mocquet, and C. Sotin. Tidal dissipation within large icy satellites: Applications to Europa and Titan. Icarus, 177(2):534 - 549, 2005. Europa Icy Shell.

D. L. Turcotte and G. Schubert. Geodynamics. Cambridge University Press, Cambridge, 2002.

E. P. Turtle, W. L. Jaeger, and P. M. Schenk. Ionian mountains and tectonics: Insights into what lies beyond Io's lofty peaks. In R.M.C. Lopez and J.R. Spencer, editors, Io after Galileo: A new view of Jupiter's volcanic moon, pages 109-131. Springer, 2007.

W. Unno, Y. Osaki, H. Ando, H. Saio, and H. Shibahashi. Nonradial oscillations of stars. Tokyo Univ. Press, Tokyo, 2 edition, 1989. 
F. W. Wagner, N. Tosi, F. Sohl, Rauer H., and T. Spohn. Rocky super-earth interiors. A 6 A, 541:A103, 2012.

F. Waleffe. On the three-dimensional instability of strained vortices. Phys. Fluids A, 2.: 76-80, 1990.

J. Wicht and A. Tilgner. Theory and modeling of planetary dynamos. Space Sci. Rev., 152:501-542, 2010.

M. Wolstencroft, J. H. Davies, and D. R. Davies. Nusselt Rayleigh number scaling for spherical shell Earth mantle simulation up to a Rayleigh number of $10^{9}$. Phys. Earth Planet. Inter., 176:132 - 141, 2009.

Y. Wu. Origin of tidal dissipation in Jupiter. I. Properties of inertial-mode. ApJ, 635: 674-687, 2005a.

Y. Wu. Origin of tidal dissipation in Jupiter. II. The value of Q. ApJ, 635:688-710, 2005b.

K. Zhang, P. Earnshaw, X. Liao, and F. H. Busse. On inertial waves in a rotating fluid sphere. JFM, 437:103-119, 2001. 


\section{List of Figures}

1.1. Jupiter and Io photographed by the Voyager 2 probe on 9 July 1979. Courtesy: NASA . . . . . . . . . . . . . . . . . . . 13

2.1. The elliptic orbit is the reason for the libration of the central object (big sphere) on its orbit around the tidal perturber (small sphere). The orbit is synchronized but not circularized. One face of the central object is always pointing at the empty focus of the ellipse, while the tidal bulge (which is not plotted here) always points to the tidal pertuber (the occupied focus) (Murray and Dermott, 1999). Therefore the red parts of the central objects can be seen from the tidal perturber, caused by the libration. On a circular orbit only the green parts would be visible from the tidal perturber (see figure in zum.de/Faecher/Materialien/gebhardt/astronomie/libration.html).

2.2. Frequencies of inertial modes in the co-rotating frame, $q$, for $\beta=0$ (cross) and $\beta=18$ (circle) as a function of the spatial degree $n$. Only the modes with $k=-1$ (green) and $k=1$ (blue) are shown. Credit: Clausen \& Tilgner, A\&A, 562, A25, 2014, reproduced with permission @ESO. . . . . .

2.3. Growth rates of modes $(-1,1)$ for various $n$ as a function of the compressibility parameter $\beta$ for $\epsilon=0.04, \Omega_{\mathrm{p}}=0$, and $E k=0$ (a). The corresponding $\Delta \omega$ (b). Credit: Clausen \& Tilgner, A\&A, 562, A25, 2014, reproduced with permission $\odot$ ESO . . . . . . . . . . . . . . . .

2.4. Same as Fig. 2.3 but for $\epsilon=0.16$. Credit: Clausen \& Tilgner, A\&A, 562, A25, 2014, reproduced with permission OESO. . . . . . . . . 30

2.5. Maximum growth rates as a function of $\Omega$ for $\mathrm{Ek}=0$. For this figure we take into account all modes with $|k| \leq 10$ and $n \leq 40$. The black line in this figure is the growth rate given by Eq. (2.50). Credit: Clausen \& Tilgner, A\&A, 562, A25, 2014, reproduced with permission @ESO . . . . . . . 31

2.6. The corresponding values of the spatial degree $n=n_{i}=n_{j}$ for the growth rates in Fig. 2.5 . . . . . . . . . . . . . . . . . . . . . 32 
2.7. Mean value of the spatial degree $\langle n\rangle$, for the modes with a growth rate of at least $0.1 \sigma_{u d}$ (dashed lines) or $0.8 \sigma_{u d}$ (solid lines) as a function of $\epsilon$ for $\beta=0$ (green $\mathrm{x}$ ), $\beta=2.8$ (red circles), and $\beta=18$ (blue squares), together with fits according to Eq.2.52. Credit: Clausen \& Tilgner, A\&A, 562, A25, 2014, reproduced with permission OESO . . . . . . . . . . . . 33

2.8. Minimum value of $n, n_{\min }$, as a function of $\Omega$ for various $\epsilon$ and positive $\Omega$. $\beta=2.8$ and $\beta=18$ (a) and $\beta=0$ (b). Credit: Clausen \& Tilgner, A\&A, 562 , A25, 2014, reproduced with permission @ESO. . . . . . . . 34

2.9. Same as in Fig. 2.8 but for negative $\Omega$. Credit: Clausen \& Tilgner, A\&A, 562, A25, 2014, reproduced with permission @ESO . . . . . . . . 34

2.10. Maximum growth rates as a function of $\Omega$ for $E k \neq 0$. For this figure we take into account all modes with a maximum $|k| \leq 10$ and $n \leq 40$. In (a) calculated for free slip boundary conditions according to Eq. (2.37). In (b) calculated for no slip boundary conditions according to Eq. (2.38). The black line in this figure is the growth rate given by Eq. (2.50). The cyan colored line in (a) is the growth rate given by Eq. (2.57) with $d_{2}=$ 3.35. Credit: Clausen \& Tilgner, A\&A, 562, A25, 2014, reproduced with permission $\mathrm{OESO} \ldots \ldots \ldots \ldots \ldots \ldots \ldots$

2.11. The corresponding values of the spatial degree $n=n_{i}=n_{j}$ for the growth rates in Fig. 2.10. . . . . . . . . . . . . . . . .

3.1. Only the rheological boundary layer, with thickness $\delta_{\mathrm{rh}}$, of the diffusive lid, with thickness $\delta_{\mathrm{d}}$, participates in the convective flow and only this part is mobile, $u_{x} \neq 0$. But also in the rheological boundary layer $u_{y}=0$, such that also across this layer heat is transported only by diffusion. Figure after Douce (2011). . . . . . . . . . . . . . . . 5

3.2. Left: Viscosity as a function of temperature; Right: Shear modulus as a function of temperature . . . . . . . . . . . . . . 59

3.3. Tidal dissipation rate $D_{\mathrm{T}}$, modulus of the tidal love number $k_{2}$ and dissipation factor $\mathrm{Q}$ versus asthenosphere thickness of Io, using parameters from Segatz et al. (1988), and equivalent to their Fig. 5. (a): own incompressible calculations; (b): own compressible calculations. . . . . . . . . . . .

3.4. Love number $h_{2}$ versus ice viscosity of Europa using parameters from Sotin et al. (2009), and equivalent to their Fig. 5. . . . . . . . . . . . . . .

3.5. Dissipation according to time dependent convection (tdC) and tidal heat $\left(D_{\mathrm{T}}\right)$ for two different rheologies in a homogenous mantle model. Model parameter see Table 3.1-3.3. $N u(R a)$ according to Eq. (3.29). Credit: Clausen \& Tilgner, A\&A, 2015, DOI: 10.1051/0004-6361/201526082, reproduced with permission $\mathrm{OESO} \ldots \ldots \ldots \ldots$ 
3.6. Tidal dissipation $\left(\mathrm{D}_{\mathrm{T}}\right)$ and transported heat assuming time dependent convection $(\mathrm{tdC})$ for three different asthenosphere thicknesses with a conductivity of $4 \mathrm{Wm}^{-1} \mathrm{~K}^{-1}$ (a) as general assumed in this study and for comparison with a conductivity of $2 \mathrm{Wm}^{-1} \mathrm{~K}^{-1}$ (b). Model parameter see Tables 3.1-3.4, if not otherwise stated in the legend the values for Model 1, M1, were used. $N u(R a)$ according to Eq. (3.29) $(\operatorname{tdC}) . B=25 \ldots \ldots 66$

3.7. As in Fig. 3.6(a) but with a $N u(R a)$ scaling according to (3.32) (a) and with the same scaling as in (3.29) but with $\varsigma=0.3$ and $\alpha=1.3$ instead of $1 / 3$ and $4 / 3(\mathrm{~b})$, the asthenosphere thicknesses can be found in the respective legend. $B=25 \ldots \ldots \ldots \ldots \ldots \ldots \ldots$

3.8. Heat transported by melt migration $(\mathrm{mm})$ for three different grain sizes, assuming time dependent convection $(\operatorname{tdC})$ and tidal heat $\left(\mathrm{D}_{\mathrm{T}}\right)$ for three different asthenosphere thicknesses and $B=25$ (a) and $B=40$ (b), model M1. See Tables 3.1-3.4 for model parameters. $N u(R a)$ according to Eq. (3.29) for time dependent convection and melt migration according to Eq. (3.50). Credit: Clausen \& Tilgner, A\&A, 2015, DOI: 10.1051/0004$6361 / 201526082$, reproduced with permission OESO. . . . . . . . . . .

3.9. The grain size $b$ (a) and the corresponding melt fraction at the top of the melting zone (b) for which a tidal dissipation of $10^{14} \mathrm{~W}$ is obtained as a function of asthenosphere thickness, calculated with a constant melt fraction in the asthenosphere ( $\phi$ const) according to Eq. (3.50) and a variable ( $\phi$ vari) as described in Sect. 3.1.2. The convective heat transport is neglected. Credit: Clausen \& Tilgner, A\&A, 2015, DOI: 10.1051/0004$6361 / 201526082$, reproduced with permission @ESO. . . . . . . . . . .

3.10. Amount of heat transported by time dependent convection $\left(D_{c}\right)$ divided by the total heat transported by convection plus melt migration $\left(D_{T}\right)$ as a function of asthenosphere thickness (a). The corresponding grain size (b) was chosen such that the value of tidal dissipation is $10^{14} \mathrm{~W}$, respectively $6 \times 10^{13} \mathrm{~W}$ for small asthenosphere thicknesses, were a value of $10^{14} \mathrm{~W}$ was not accessible. The M1 model was used, model parameter see Tables 3.1-3.4. $N u(R a)$ according to Eq. (3.29) for time dependent convection and melt migration according to Eq. (3.50). Credit: Clausen \& Tilgner, A\&A, 2015, DOI: 10.1051/0004-6361/201526082, reproduced with permission @ESO. . . 70 
3.11. $Q^{\prime}$ as a function of eccentricity calculated with depth dependent viscosity as described in Sect. 3.1.2, for a planet with the size and density of Corot $7 \mathrm{~b}$ and four different asthenosphere thicknesses. The contribution by a convective heat transport is not included for the calculation of $Q^{\prime}$. Two different orbital periods $P=2 \mathrm{~d}$ (a) and $P=6 \mathrm{~d}$ (b) were considered. If not otherwise stated $b=5 \mathrm{~mm}$ and $B=40$. Model parameter see Tables 3.1 and 3.3-3.5. Credit: Clausen \& Tilgner, A\&A, 2015, DOI: 10.1051/00046361/201526082, reproduced with permission @ESO.

3.12. (a) As in Fig 3.11 but for a planet with the size and density of Io. The respective orbital period is quoted in the legend. See Tables 3.1-3.4 for model parameters. (b) Q' for stagnant lid convection (sl) and plate tectonic convection (pt) as the dominant heat transport mechanism as a function of the eccentricity, for a planet with the size and density of Corot $7 \mathrm{~b}$. Credit: Clausen \& Tilgner, A\&A, 2015, DOI: 10.1051/0004-6361/201526082, reproduced with permission $\mathrm{OESO} \ldots \ldots \ldots \ldots \ldots$

4.1. Real (red solid line) and imaginary part (green dashed line) of the velocity component $\hat{w}$ as a function of height for the analytical calculation (left) and the numerical calculation according to Eq. (4.12) (right). Wave parameters are $k_{x}=3, k_{y}=1$ and $\omega \approx 0.611$ and it is $U=0.1$ and for the numerical

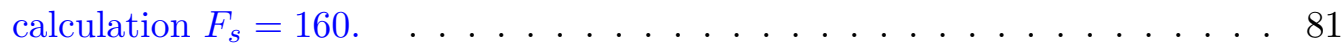

4.2. Real (red solid line) and imaginary part (green dashed line) of the velocity component $\hat{w}$ as a function of height for the numerical calculation according to the Eq. eqv, displayed in Fig. F.1. Parameters as in Fig 4.1. . . . . . . .

4.3. Real (red solid line) and imaginary part (green dashed line) of $c_{3}$, Eq. (4.34), as a function of height for the numerical calculation according to the Eq. eqv, displayed in Fig. F.1, (left) and the real and imaginary part of the corresponding pressure $\hat{p}$ according to Eq. pw, displayed in Fig. F.1, (right). Parameters as in Fig 4.1. . . . . . . . . . . . . . 83

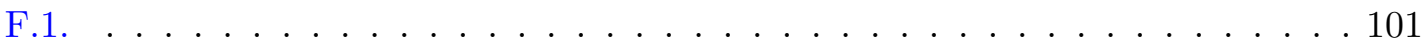




\section{List of Tables}

2.1. Parameters for the example objects. $\nu_{\mathrm{m}}$ is the molecular viscosity, $E k_{\mathrm{m}}$ the Ekman number based only on the molecular viscosity, and $E k_{\text {turb;NR }}$ and $E k_{\text {turb;RC }}$ the Ekman number based on the turbulent viscosity without and with the effects of rotation on convection, respectively, (the molecular viscosity is negligible in comparison to the turbulent in these examples). $\chi_{\mathrm{m}}, \chi_{\text {turb;NR }}$, and $\chi_{\text {turb;RC }}$ are damping constants calculated with $E k_{\mathrm{m}}$, $E k_{\text {turb;NR }}$, and $E k_{\text {turb;RC }}$, respectively. n.s. and f.s. stands for no slip and free slip boundary conditions, respectively. . . . . . . . . . . . . . 41

2.2. Summary of the stability characteristics of the flow for the examples JupiterIo (J-I) and V636 Centauri (VC). The top row indicates if turbulent viscosity without the effects of rotation on the convection has been used $\left(\nu_{\mathrm{t} ; \mathrm{NR}}\right)$ or with these effects $\left(\nu_{\mathrm{t} ; \mathrm{RC}}\right)$ in the computation of the viscous damping. We used free slip boundary conditions. . . . . . . . . . . . . . . . . 44

3.1. Notation and Parameters which are treated as constants in this study. . . . 62

3.2. Notation and parameters for Io. . . . . . . . . . . . . . . . . . . . 62

3.3. Parameter values for the rheology model according to Fischer and Spohn

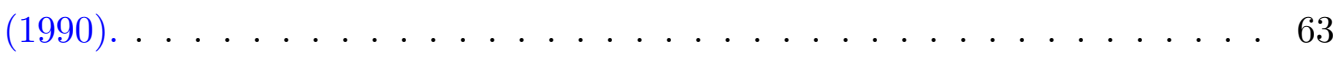

3.4. M1 values according to Segatz et al. (1988) and M2 with a lower shear modulus and viscosity in the deep mantle. For the lithosphere thickness see also Turtle et al. (2007) . . . . . . . . . . . . . . . . . . . . . 64

3.5. Notation and parameters for Corot $7 \mathrm{~b}$. For the planetary albedo we assume a typical value for terrestrial planets. For the deep mantle viscosity we assume a higher value as in Io as expected for a super earth (Tackley et al., 2013) . . . . . . . . . . . . . . . . . . . 72 


\section{Acknowledgments}

First of all, I would like to express my gratitude to my advisor Professor Andreas Tilgner for giving me the opportunity to perform this thesis and his support during my years at the Institute of Geopyhsics. For me it was always a pleasure to work with him.

This thesis was funded by the Deutsche Forschungsgemeinschaft (DFG) under grant SFB 963/1, project A5, thanks a lot to the DFG for creating this SFB and the financial support.

I would like to thank my second supervisor Professor Stefan Dreizler for his interest in this thesis and for agreeing to act as a second examiner.

I would also like to thank all current and former members of the Institute of Geophysics for interesting discussions and a supportive, friendly working atmosphere.

Furthermore, I want to thank Antoinette Solomon and Natascha Tetzlaff, who helped me to revise this thesis for the final version.

Finally, I would thank my friends and my family, especially my parents, for the support and nice hours outside of work. 